JOURNAL OF THE

AMERICAN MATHEMATICAL SOCIETY

Volume 12, Number 2, April 1999, Pages 569-618

S 0894-0347(99)00293-3

\title{
VOLUMES OF HYPERBOLIC MANIFOLDS AND MIXED TATE MOTIVES
}

\author{
ALEXANDER GONCHAROV
}

\section{Contents}

1. Introduction $\quad 569$

2. Hyperbolic volumes and Borel's regulator 577

3. Noneuclidean polyhedrons and mixed Hodge structures 587

4. Periods 596

5. $\quad$ Mixed Tate motives over a number field and Theorem $1.6 \quad 603$

6. Remarks on Dehn complexes 613

$\begin{array}{ll}\text { Acknowledgements } & 616\end{array}$

$\begin{array}{ll}\text { References } & 616\end{array}$

\section{INTRODUCTION}

By a hyperbolic manifold we mean an orientable complete Riemannian manifold with constant sectional curvature -1 .

1. Volumes of $(2 n-1)$-dimensional hyperbolic manifolds and the Borel regulator on $K_{2 n-1}(\overline{\mathbb{Q}})$. Let $M^{n}$ be an $n$-dimensional hyperbolic manifold with finite volume $\operatorname{vol}\left(M^{n}\right)$. If $n=2 m$ is an even number, then by the Gauss-Bonnet theorem $([\mathrm{Ch}])$

$$
\operatorname{vol}\left(M^{2 m}\right)=-c_{2 m} \cdot \chi\left(M^{2 m}\right)
$$

where $c_{2 m}=1 / 2 \times\left(\right.$ volume of sphere $S^{2 m}$ of radius 1$)$ and $\chi\left(M^{2 m}\right)$ is the Euler characteristic of $M^{2 m}$. This is straightforward for compact manifolds and a bit more delicate for noncompact ones.

According to Mostow's rigidity theorem (see [Th], Ch. 5) volumes of hyperbolic manifolds are homotopy invariants. For even-dimensional ones this is clear from the formula above. The volumes of odd-dimensional hyperbolic manifolds form a very interesting set of numbers. If the dimension is $\geq 5$ it is discrete and, moreover, for any given $c \in \mathbb{R}$ there is only a finite number of hyperbolic manifolds of volume $\leq c$ ([W]). Thanks to Jorgensen and Thurston we know that the volumes of hyperbolic 3 -folds form a nondiscrete well-odered set of ordinal type $\omega^{\omega}$ (see [Th]).

We will denote by $\overline{\mathbb{Q}}$ the subfield of all algebraic numbers in $\mathbb{C}$.

There is the Borel regulator $[\mathrm{Bo} 2] r_{m}: K_{2 m-1}(\mathbb{C}) \rightarrow \mathbb{R}$.

Received by the editors October 16, 1996 and, in revised form, October 11, 1998.

1991 Mathematics Subject Classification. Primary 11Gxx; Secondary 19Fxx, 57-XX. 
Theorem 1.1. Any $(2 m-1)$-dimensional hyperbolic manifold of finite volume $M^{2 m-1}$ defines naturally an element $\gamma\left(M^{2 m-1}\right) \in K_{2 m-1}(\overline{\mathbb{Q}}) \otimes \mathbb{Q}$ such that $\operatorname{vol}\left(M^{2 m-1}\right)=r_{m}\left(\gamma\left(M^{2 m-1}\right)\right)$.

For an abelian group $A$ set $A_{\mathbb{Q}}:=A \otimes \mathbb{Q}$. Let $\varepsilon(n)$ be the abelian group $\mathbb{Z}$ equipped with the action of $\operatorname{Gal}(\mathbb{C} / \mathbb{R})$ such that the complex conjugation acts by $(-1)^{n}$. In Sections 3 and 5 we describe a different construction of an invariant

$$
\tilde{\gamma}\left(M^{2 n-1}\right)^{-} \in\left(K_{2 n-1}(\overline{\mathbb{Q}})_{\mathbb{Q}} \otimes \varepsilon(n)\right)^{-}
$$

which depends only on the scissors congruence class of a hyperbolic manifold. This means that if two hyperbolic manifolds can be cut on the same geodesic simplices, then their invariants $\tilde{\gamma}\left(M^{2 n-1}\right)^{-}$coincide. We will prove that Theorem 1.1 remains valid if we replace $\gamma\left(M^{2 n-1}\right)$ by $\tilde{\gamma}\left(M^{2 n-1}\right)^{-}$. This gives a completely different and more conceptual proof of this theorem. One should have $\tilde{\gamma}\left(M^{2 n-1}\right)^{-}=\gamma\left(M^{2 n-1}\right)^{-}$.

2. Applications: The classical polylogarithms and volumes of hyperbolic $(2 n-1)$-manifolds. Theorem 1.1 together with explicit computation of the Borel regulator for $K_{3}(\mathbb{C})([\mathrm{Bl} 1])$ and $K_{5}(\mathbb{C})([\mathrm{G} 2])$ leads to much more precise results about the volumes of hyperbolic 3 - and 5 -manifolds.

Let us recall the classical polylogarithms, defined inductively as multivalued analytical functions on $\mathbb{C P}^{1} \backslash\{0,1, \infty\}$ :

$$
L i_{n}(z):=\int_{0}^{z} L i_{n-1}(w) \frac{d w}{w}, \quad L i_{1}(z)=-\log (1-z) .
$$

The function $\log z$ has a single-valued cousin $\log |z|=\operatorname{Re} \log z$. For arbitrary $n$ there is the following single-valued function [Z1]:

$$
\mathcal{L}_{n}(z):=\mathcal{R}_{n}\left(\sum_{k=1}^{n} \frac{B_{k} \cdot 2^{k}}{k !} L i_{n-k}(z) \cdot \log ^{k}|z|\right) .
$$

Here $\mathcal{R}_{n}$ means the real part for $n$ odd and the imaginary part for $n$ even, and $B_{k}$ are Bernoulli numbers: $\sum_{k=0}^{\infty} \frac{B_{k} \cdot 2^{k}}{k !} \cdot x^{k}=\frac{2 x}{e^{2 x}-1}$. It is continuous on $\mathbb{C P}^{1}$ (and, of course, real analytic on $\left.\mathbb{C P}^{1} \backslash\{0,1, \infty\}\right)$. For example,

$$
\begin{gathered}
\mathcal{L}_{2}(z):=\operatorname{Im} L i_{2}(z)+\arg (1-z) \cdot \log |z|, \\
\mathcal{L}_{3}(z):=\operatorname{Re}\left(L i_{3}(z)-\log |z| \cdot L i_{2}(z)+\frac{1}{3} \log ^{2}|z| \cdot L i_{1}(z)\right) .
\end{gathered}
$$

Theorem 1.2. Let $M^{3}$ be a hyperbolic 3-manifold of finite volume. Then there exist numbers $z_{i} \in \overline{\mathbb{Q}}$ such that

$$
\sum\left(1-z_{i}\right) \wedge z_{i}=0 \text { in } \Lambda^{2} \overline{\mathbb{Q}}^{*} \quad \text { and } \quad \operatorname{vol}\left(M^{3}\right)=\sum \mathcal{L}_{2}\left(z_{i}\right)
$$

This theorem follows also from the results of Dupont-Sah ([Du1], [DS1]), or Neumann-Zagier [NZ].

Let $X$ be a set. Denote by $\mathbb{Z}[X]$ the free abelian group generated by symbols $\{x\}$ where $x$ runs through all elements of $X$.

Let $r(\cdot, \cdot, \cdot, \cdot)$ be the cross-ratio of 4 distinct points on a line. Let $F$ be a field and let $R_{2}(F) \subset \mathbb{Z}\left[P_{F}^{1} \backslash\{0,1, \infty\}\right]$ be the subgroup generated by the elements $\sum_{i=1}^{5}(-1)^{i}\left\{r\left(x_{1}, \ldots, \hat{x}_{i}, \ldots, x_{5}\right)\right\}\left(x_{i} \neq x_{j} \in P_{F}^{1}\right)$. Set

$$
B_{2}(F)=\frac{\mathbb{Z}\left[P_{F}^{1} \backslash\{0,1, \infty\}\right]}{R_{2}(F)} .
$$


Denote by $\{x\}_{2}$ the projection of $\{x\}$ onto $B_{2}(F)$.

Theorem 1.3. Let $M^{5}$ be a 5-dimensional hyperbolic manifold of finite volume. Then there exist algebraic numbers $z_{i} \in \overline{\mathbb{Q}}$ satisfying the condition

$$
\sum_{i}\left\{z_{i}\right\}_{2} \otimes z_{i}=0 \text { in } B_{2}(\overline{\mathbb{Q}}) \otimes \overline{\mathbb{Q}}^{*} \quad \text { and } \quad \operatorname{vol}\left(M^{5}\right)=\sum_{i} \mathcal{L}_{3}\left(z_{i}\right) .
$$

One can define for arbitrary $n$ a certain subgroup $\mathcal{R}_{n}(F) \subset \mathbb{Z}\left[\mathbb{P}_{F}^{1}\right]$ which for $F=\mathbb{C}$ is the subgroup of all functional equations for the $n$-logarithm $\mathcal{L}_{n}(z)$ (see s. 1.4 of $[\mathrm{G} 2])$. Let $\mathcal{B}_{n}(F):=\mathbb{Z}\left[\mathbb{P}_{F}^{1}\right] / \mathcal{R}_{n}(F)$. One has $\mathcal{B}_{2}(F)=B_{2}(F)$. Define the homomorphism

$$
\delta_{n}: \mathbb{Z}\left[\mathbb{P}_{F}^{1}\right] \rightarrow \mathcal{B}_{n-1}(F) \otimes F^{*}, \quad\{x\} \mapsto\{x\}_{n-1} \otimes x .
$$

One can show that $\delta_{n}\left(\mathcal{R}_{n}(F)\right)=0([\mathrm{G} 2])$, so we get a homomorphism $\delta_{n}: \mathcal{B}_{n}(F) \rightarrow$ $\mathcal{B}_{n-1}(F) \otimes F^{*}$.

Conjecture 1.4. Let $M^{2 n-1}$ be a $(2 n-1)$-dimensional hyperbolic manifold of finite volume. Then there exist algebraic numbers $z_{i} \in \overline{\mathbb{Q}}$ such that $(n \geq 3)$

$$
\sum_{i}\left\{z_{i}\right\}_{n-1} \otimes z_{i}=0 \text { in } \mathcal{B}_{n-1}(\overline{\mathbb{Q}}) \otimes \overline{\mathbb{Q}}^{*} \quad \text { and } \quad \operatorname{vol}\left(M^{2 n-1}\right)=\sum_{i} \mathcal{L}_{n}\left(z_{i}\right) .
$$

Theorem 1.3 (as well as Theorem 1.2) follows from Theorem 1.1 and the main results of [G2]. Conjecture 1.4 is a consequence of Zagier's conjecture [Z1] and Theorem 1.1.

3. The invariants $\gamma\left(M^{2 n-1}\right)$ and $\tilde{\gamma}\left(M^{2 n-1}\right)^{-}$. There are two completely different points of view on algebraic K-theory.

i) In Quillen's definition, $K_{n}(F)_{\mathbb{Q}}$ is the quotient of $H_{n}(G L(F), \mathbb{Q})$ modulo the subspace of decomposable elements. This is how elements $\gamma\left(M^{2 m-1}\right)$ will be constructed in Section 2. The construction is straightforward for 3-dimensional compact hyperbolic manifolds: the fundamental class of such a manifold provides an element in $H_{3}(B S O(3,1), \mathbb{Z})$; using the local isomorphism between $S O(3,1)$ and $S L(2, \mathbb{C})$ we get a class in $H_{3}\left(S L(2, \mathbb{C})^{\delta}, \mathbb{Q}\right)$ and thus an element in $H_{3}\left(S L(\mathbb{C})^{\delta}, \mathbb{Q}\right)$ via the embedding $S L_{2} \hookrightarrow S L$. It is interesting that for compact hyperbolic manifolds of dimension $\geq 5$ one has to use the half-spinor representation of $S O(2 n-1,1)$ in order to get, starting from the fundamental class of $M^{2 n-1}$, an interesting class in $H_{2 n-1}(G L(\mathbb{C})$ ). (It seems that the other fundamental representations of $S O(2 n-1,1)$ lead to zero classes in $\left.K_{2 n-1}(\mathbb{C})\right)$. For the noncompact hyperbolic manifolds, $H_{2 n-1}\left(M^{2 n-1}\right)=0$, and the construction of the invariant $\gamma\left(M^{2 n-1}\right)$ becomes rather delicate; see Section 2.

ii) Recall the $\gamma$-filtration on $K$ groups. According to A.A. Beilinson for an arbitrary field $F$ one should have

$$
g r_{n}^{\gamma} K_{2 n-i}(F) \otimes \mathbb{Q}=\operatorname{Ext}_{\mathcal{M}_{F}}^{i}(\mathbb{Q}(0), \mathbb{Q}(n)) .
$$

Here $\mathcal{M}_{F}$ is the hypothetical abelian category of mixed motives over a field $F$ and $\mathbb{Q}(n)$ is the $n$-th tensor power of the Tate motive $\mathbb{Q}(1)$; the latter is the inverse to the motive $\mathbb{Q}(-1):=H^{2}\left(\mathbb{C P}^{1}\right)$. It should contain the abelian tensor subcategory $\mathcal{M}_{T}(F)$, which is the smallest subcategory containing the Tate motives $\mathbb{Q}(n)$ and closed under extensions.

If $F$ is a number field, such an abelian category $\mathcal{M}_{T}(F)$ is already available, and moreover the $E x t$ 's between $\mathbb{Q}(0)$ and $\mathbb{Q}(n)$ in this category are given by formula 
(1) (where $\mathcal{M}_{F}$ is replaced by $\mathcal{M}_{T}(F)$ ). This follows from the existence of a certain triangulated (not abelian!) category of mixed motives over a field $F$ constructed independently by Hanamura $[\mathrm{H}]$, Levine $[\mathrm{L}]$, and Voevodsky $[\mathrm{V}]$ in which the Extgroups are isomorphic to the needed parts of the K-theory, some formal arguments, and the Borel theorem; see Section 5.

For any hyperbolic manifold of finite volume $M^{2 n-1}$ we construct an extension $m\left(M^{2 n-1}\right) \in \operatorname{Ext}_{\mathcal{M}_{\overline{\mathbb{Q}}}}^{1}(\mathbb{Q}(0), \mathbb{Q}(n))$ in the category of mixed Tate motives over $\overline{\mathbb{Q}}$ such that the $\mathbb{R}$-part of the regulator map on this class coincides with the volume of $M^{2 n-1}$. Namely, we produce an extension in the category of mixed Hodge structures which is clearly of algebraic-geometric origin. Below we outline a more general framework in which these ideas works.

4. The scissors congruence groups and the Dehn invariant. Let $V^{n}$ be an $n$-dimensional space with one of the three classical geometries, i.e. $V$ is the hyperbolic space $\mathcal{H}^{n}$, spherical space $S^{n}$ or Euclidean space $E^{n}$.

The scissors congruence group $\mathcal{P}\left(V^{m}\right)$ is an abelian group generated by pairs $[M, \alpha]$ where $M$ is an oriented geodesic simplex and $\alpha$ is an orientation of $V^{m}$. The relations are: $[M, \alpha]=\left[M_{1}, \alpha\right]+\left[M_{2}, \alpha\right]$ if $M=M_{1} \cup M_{2} ;[M, \alpha]$ changes sign if we change orientation of $M$ or $\alpha$, and $[M, \alpha]=[g M, g \alpha]$ for any $g$ in the symmetry group of $V^{m}$. One can show that $\mathcal{P}\left(\mathcal{S}^{2 k}\right)=0$.

The volume of a geodesic simplex provides homomorphisms

$$
\operatorname{vol}_{\mathcal{H}}: \mathcal{P}\left(\mathcal{H}^{n}\right) \rightarrow \mathbb{R}, \quad \operatorname{vol}_{S}: \mathcal{P}\left(S^{2 n-1}\right) \rightarrow \mathbb{R} / \mathbb{Z}, \quad \operatorname{vol}_{E}: \mathcal{P}\left(E^{n}\right) \rightarrow \mathbb{R} .
$$

It is not hard to see (and in the euclidean case was known to Euclid) that the volume provides isomorphisms

$$
\mathcal{P}\left(S^{1}\right)=\mathbb{R} / \mathbb{Z}, \quad \mathcal{P}\left(\mathcal{H}^{1}\right)=\mathcal{P}\left(\mathcal{H}^{2}\right)=\mathbb{R}, \quad \mathcal{P}\left(E^{1}\right)=\mathcal{P}\left(E^{2}\right)=\mathbb{R} .
$$

The Hilbert Third Problem. Determine the structure of scissors congruence groups in classical geometries.

In fact Hilbert himself asked the following question: Is it true that the volume homomorphism is injective in dimension three? The negative answer was given by Max Dehn (1898). He discovered that in dimension $\geq 3$ the volume does not separate the elements of the scissors congruence group, because a new phenomenon appears: the Dehn invariant. It is a homomorphism

$$
D_{n}^{V}: \mathcal{P}\left(V^{n}\right) \longrightarrow \bigoplus_{i=1}^{n-2} \mathcal{P}\left(V^{i}\right) \otimes \mathcal{P}\left(S^{n-i-1}\right)
$$

defined as follows. Each $i$-dimensional face $A$ of $M$ can be viewed as a geodesic simplex $v(A)$ in $V^{i}$. In the orthogonal plane $A^{\perp}$ the simplex $M$ always cuts a spherical simplex $s(A)$. Choose orientations $\alpha_{A}$ and $\beta_{A}$ of $A$ and $A^{\perp}$ such that $\alpha_{A} \otimes \beta_{B}=\alpha$. Then $D_{n}^{V}([M, \alpha]):=\sum_{A}\left[v(A), \alpha_{A}\right] \otimes\left[s(A), \beta_{A}\right]$. For instance using (2) we get homomorphisms

$$
D_{3}^{h}: \mathcal{P}\left(\mathcal{H}^{3}\right) \longrightarrow \mathbb{R} \otimes \mathbb{R} / \mathbb{Z}, \quad D_{3}^{s}: \mathcal{P}\left(S^{3}\right) \longrightarrow \mathbb{R} / \mathbb{Z} \otimes \mathbb{R} / \mathbb{Z} .
$$

A hyperbolic manifold of finite volume can be cut on geodesic simplices and so produces an element in $\mathcal{P}\left(\mathcal{H}^{2 n-1}\right)$. Its Dehn invariant is equal to zero. 
5. Kernel of the Dehn invariant in noneuclidean geometry and $K_{2 n-1}(\overline{\mathbb{Q}}) \otimes$ $\mathbb{Q}$. Our key idea is that any geodesic simplex $M$ in spherical or hyperbolic geometry defines a mixed Tate motive, and moreover the Dehn invariant also has a motivic interpretation.

The Klein model of $\mathcal{H}^{m}$ is the interior of a ball in $\mathbb{R}^{m}$ and geodesics are straight lines. The distance $\rho\left(P_{1}, P_{2}\right)$ is defined as $\left|\log r\left(Q_{1}, Q_{2}, P_{1}, P_{2}\right)\right|$ where $Q_{1}, Q_{2}$ are the intersection points of the line $P_{1} P_{2}$ with the absolute: the sphere $Q$. So a geodesic simplex is the usual one inside the absolute: the sphere $Q$. After complexification and compactification we get $\mathbb{C P}^{m}$ together with a quadric $Q$ and a collection of hyperplanes $M=\left(M_{1}, \ldots, M_{m+1}\right)$ provided by the faces of the simplex.
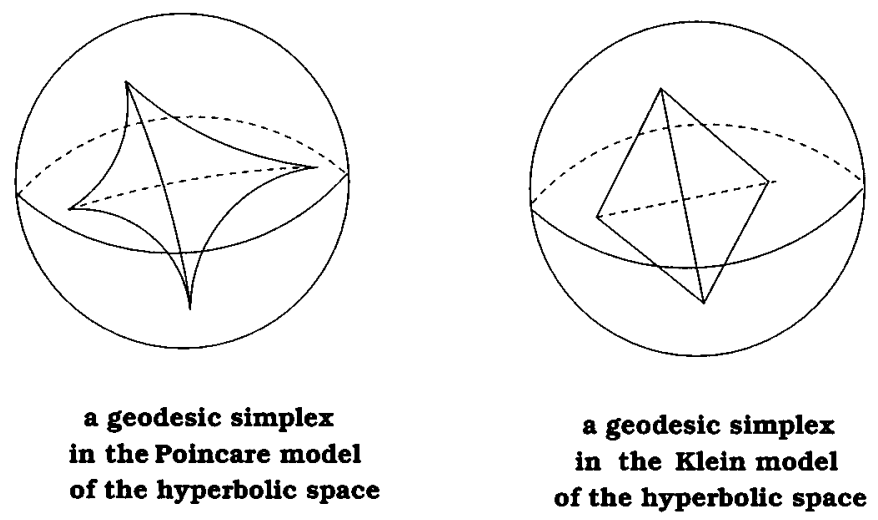

Similarly a quadric $Q \in \mathbb{C P}^{m}$ without points in $\mathbb{R} \mathbb{P}^{m}$ defines an elliptic geometry in $\mathbb{R P}^{m}$; the geodesic simplices are just the usual ones.

Now suppose that $m=2 n-1$. Let $h(Q, M)$ be the mixed Hodge structure on $H^{2 n-1}\left(\mathbb{C P}^{2 n-1} \backslash Q, M ; \mathbb{Q}\right)$. It is equipped with the following additional structures. There is a canonical, up to a sign, meromorphic $2 n-1$-form $\omega_{Q}$ in $\mathbb{C P}^{2 n-1} \backslash Q$ with polar singularity along $Q$. If we choose coordinates $x_{1}, x_{2}, \ldots, x_{2 n}$ in $\mathbb{C}^{2 n}$ and an equation $\tilde{Q}=\sum_{i, j} q_{i j} x_{i} x_{j}=0$ of the quadric $Q$, then

$$
\omega_{Q}:= \pm i^{n} \cdot \sqrt{\operatorname{det} \tilde{Q}} \cdot \frac{\sum_{i=1}^{2 n}(-1)^{i} x_{i} d x_{0} \wedge \ldots \wedge \hat{d} x_{i} \wedge d x_{2 n-1}}{\tilde{Q}(x)^{n}} .
$$

It does not depend on the choice of the coordinates $x_{i}$ and the equation $\tilde{Q}$. The sign is provided by an orientation of $\mathcal{H}^{2 n-1}$. Let $\Delta_{M}$ be a relative cycle representing a generator of $H_{2 n-1}\left(\mathbb{C P}^{2 n-1}, M\right)$.

A mixed Hodge structure is called a Hodge-Tate structure if its Hodge numbers $h^{p, q}$ are zero unless $p=q$. A Hodge-Tate structure $H$ is said to be $n$-framed if we choose nonzero vectors $v_{n} \in g r_{2 n}^{W} H$ and $f_{0} \in\left(g r_{0}^{W} H\right)^{*}$. Appropriately defined equivalence classes of $n$-framed mixed Hodge-Tate structures form a $\mathbb{Q}$-vector space $\mathcal{H}_{n}$. Moreover, $\mathcal{H}_{\bullet}:=\bigoplus_{n=0}^{\infty} \mathcal{H}_{n}$ has a natural structure of a graded commutative Hopf algebra with the coproduct $\nu$ ([BGSV], see s.2.2).

So $h(Q, M)$ is a Hodge-Tate structure $n$-framed by $\left[\omega_{Q}\right] \in H^{2 n-1}\left(\mathbb{C P}^{2 n-1} \backslash Q\right)=$ $g r_{2 n}^{W} h(Q, M)$ and $\left[\Delta_{M}\right] \in H_{2 n-1}\left(\mathbb{C P}^{2 n-1}, M\right)=\left(g r_{0}^{W} h(Q, M)\right)^{*}$. (Compare with [BGSV].) Set $\tilde{\nu}(x):=\nu(x)-1 \otimes x-x \otimes 1$.

Theorem 1.5. a) The map $[(Q, M), \alpha] \rightarrow\left(h(Q, M),\left[\omega_{Q}\right],\left[\Delta_{M}\right]\right) \in \mathcal{H}_{n}$ provides homomorphisms of groups $\mu_{h}: \mathcal{P}\left(\mathcal{H}^{2 n-1}\right) \rightarrow \mathcal{H}_{n}$ and $\mu_{s}: \mathcal{P}\left(S^{2 n-1}\right) \rightarrow \mathcal{H}_{n}$. 
b) The following diagram is commutative:

$$
\begin{array}{cccc}
\mathcal{P}\left(\mathcal{H}^{2 n-1}\right) & \stackrel{D_{n}^{h}}{\longrightarrow} & \bigoplus_{k} \mathcal{P}\left(\mathcal{H}^{2 k-1}\right) \otimes \mathcal{P}\left(S^{2(n-k)-1}\right) \\
\downarrow \mu_{h} & & \downarrow \mu_{h} \otimes \mu_{s} \\
\mathcal{H}_{n} & \stackrel{\tilde{\nu}}{\longrightarrow} & \bigoplus_{k} \mathcal{H}_{k} \otimes \mathcal{H}_{n-k}
\end{array}
$$

There is a similar diagram for the spherical Dehn invariant.

This theorem provides a Hodge-theoretic (and in fact motivic) interpretation of the Dehn invariant in noneuclidean geometries.

If a pair $(Q, M)$ corresponds to a geodesic simplex in $\mathcal{H}^{2 n-1}$, then there is a natural choice for the relative cycle $\Delta_{M}$ such that

$$
\int_{\Delta_{M}} \omega_{Q}=\operatorname{vol}(M)
$$

There is a similar interpretation of the volume of a spherical geodesic simplex. So the volume of an odd-dimensional geodesic simplex is a period of the corresponding mixed Hodge structure. This explains why the dilogarithm appears in Lobachevsky's computation of volumes of geodesic 3-simplices (see [M1]).

Notice that in this normalization the volume of the sphere is equal to $(2 \pi i)^{n} /$ $2^{n-1}(n-1)$ !, so this normalization is different from the one we used in s. 1.4.

Let $\mathcal{P}\left(\mathcal{H}^{2 n-1}, \overline{\mathbb{Q}}\right)$ (respectively $\mathcal{P}\left(S^{(2 n-1)}, \overline{\mathbb{Q}}\right)$ ) be the subgroup of the hyperbolic (resp. spherical) scissors congruence group generated by the simplices defined over $\overline{\mathbb{Q}}$ (i.e. their vertices have coordinates in $\overline{\mathbb{Q}}$ ).

Theorem 1.6. There are the following commutative diagrams:

$$
\begin{aligned}
& \left.\operatorname{Ker} D_{2 n-1}^{H}\right|_{\mathcal{P}\left(\mathcal{H}^{2 n-1}, \overline{\mathbb{Q}}\right)} \otimes \mathbb{Q} \quad \longrightarrow \quad\left(K_{2 n-1}(\overline{\mathbb{Q}})_{\mathbb{Q}} \otimes \varepsilon(n)\right)^{-} \\
& \text {vol \} \swarrow r _ { n } } \\
{i^{n-1} \mathbb{R}} \\
{\left.\operatorname{Ker} D_{2 n-1}^{S}\right|_{\left.\mathcal{P}\left(S^{2 n-1}\right), \overline{\mathbb{Q}}\right)} \otimes \mathbb{Q} \quad \longrightarrow \quad\left(K_{2 n-1}(\overline{\mathbb{Q}})_{\mathbb{Q}} \otimes \varepsilon(n)\right)^{+}} \\
{\text {vol \} \quad \swarrow r _ { n } ^ { B e } } \\
{i^{n} \mathbb{R} /(2 \pi i)^{n} \mathbb{Q}}
\end{array}
\end{aligned}
$$

The idea is this. Let $M H S / \mathbb{Q}$ be the category of $\mathbb{Q}$-mixed Hodge structures. One has

$$
\operatorname{Ker}\left(\tilde{\nu}: \mathcal{H}_{n} \longrightarrow \bigoplus_{k+l=n ; k, l>0} \mathcal{H}_{k} \otimes \mathcal{H}_{l}\right)=\operatorname{Ext}_{M H S / \mathbb{Q}}^{1}(\mathbb{Q}(0), \mathbb{Q}(n)) .
$$

By Theorem 1.5 any element of $\mathcal{P}\left(\mathcal{H}^{2 n-1}, \overline{\mathbb{Q}}\right)$ with the Dehn invariant equal to zero defines an element of (6) of algebraic geometric origin, and thus a motivic extension of $\mathbb{Q}(0)$ by $\mathbb{Q}(n)$, which gives an element in $K_{2 n-1}(\overline{\mathbb{Q}}) \otimes \mathbb{Q}$ by $(1)$.

Conjecture 1.7. There exist canonical injective homomorphisms

$$
K e r D_{2 n-1}^{H} \otimes \mathbb{Q} \hookrightarrow\left(g r_{n}^{\gamma} K_{2 n-1}(\mathbb{C})_{\mathbb{Q}} \otimes \varepsilon(n)\right)^{-},
$$




$$
K e r D_{2 n-1}^{S} \otimes \mathbb{Q} \hookrightarrow\left(g r_{n}^{\gamma} K_{2 n-1}(\mathbb{C})_{\mathbb{Q}} \otimes \varepsilon(n)\right)^{+}
$$

such that the composition of (7) (resp. (8)) with $i^{n} \times$ Borel's (resp. $i^{n} \mathbb{R} /(2 \pi i)^{n} \mathbb{Q}$ part of Beilinson's) regulator coincide with the volume homomorphism.

(According to the rigidity conjecture the natural map $K_{2 n-1}(\overline{\mathbb{Q}}) \rightarrow g r_{n}^{\gamma} K_{2 n-1}(\mathbb{C})$ should be an isomorphism modulo torsion.)

If $n=2$, these homomorphisms exist and are isomorphisms. This follows from the beautiful results of J. Dupont and S. H. Sah (see [Du1], [DS1], [DPS], [Sah3]). Unfortunately their methods use essentially classical isomorphisms between simple Lie groups in low dimensions (like the local isomorphism between $S O(3,1)_{0}$ and $S L_{2}(\mathbb{C})$ ) and a lot of arguments "ad hoc" that one does not see how to generalize to higher dimensions, even hypothetically. The relation with algebraic K-theory was quite mysterious. After motivic interpretation of the Dehn invariant the algebraic K-theory shows up immediately thanks to (1).

Generalizing Milnor's conjecture about the values of the Lobachevsky function Ramakrishnan conjectured (see 7.1.2 in [R]) that Beilinson's regulator is injective modulo torsion. If this together with Conjecture 1.7 would have been proved, we get an affirmative answer in hyperbolic and spherical geometry to the following part of the Hilbert Third Problem:

Do the Dehn invariant and the volume separate all points of scissors congruence groups?

6. Dehn complexes in noneuclidean geometries and algebraic K-theory of $\mathbb{C}$. What about the cokernel of the Dehn invariant? The Dehn invariant in dimensions $\geq 5$ is only the beginning of a certain complex, which I will call the Dehn complex:

$$
\begin{array}{r}
\mathcal{P}_{V}^{\bullet}(n) \quad: \mathcal{P}\left(V^{2 n-1}\right) \stackrel{d_{v}}{\longrightarrow} \bigoplus_{i_{1}+i_{2}=2 n-1} \mathcal{P}\left(V^{i_{1}}\right) \otimes \mathcal{P}\left(S^{i_{2}-1}\right) \stackrel{d_{v}}{\longrightarrow} \ldots \\
\stackrel{d_{v}}{\longrightarrow} \bigoplus_{i_{1}+\ldots+i_{k}=2 n-1} \mathcal{P}\left(V^{i_{1}}\right) \otimes \mathcal{P}\left(S^{i_{2}-1}\right) \otimes \ldots \otimes \mathcal{P}\left(S^{i_{k}-1}\right) \stackrel{d_{v}}{\longrightarrow} \ldots .
\end{array}
$$

Here $V$ is one of the three geometries and the first group placed in degree 1 . The differential has degree +1 and is defined as follows:

$$
d_{v}=D^{V} \otimes i d \otimes \ldots \otimes i d-i d \otimes D^{S} \otimes i d \otimes \ldots \otimes i d+\ldots \pm \otimes i d \otimes \ldots \otimes i d \otimes D^{S} .
$$

Remark. The spherical Dehn invariant provides $\bigoplus \mathcal{P}\left(S^{2 j-1}\right)$ with a structure of a coalgebra. Further, the Dehn invariant provides $\bigoplus \mathcal{P}\left(V^{2 i-1}\right)$ with a structure of a comodules over this coalgebra. The complex above is the cobar complex computing the degree $n$ part of the cohomology of the coalgebra $\bigoplus \mathcal{P}\left(S^{2 n-1}\right)$ with coefficients in the comodule $\bigoplus \mathcal{P}\left(V^{2 n-1}\right)$.

Conjecture 1.8. There are canonical homomorphisms

$$
\begin{aligned}
& H^{i}\left(\mathcal{P}_{\mathcal{H}}^{\bullet}(n)\right) \longrightarrow\left(g r_{n}^{\gamma} K_{2 n-i}(\mathbb{C})_{\mathbb{Q}} \otimes \varepsilon(n)\right)^{-}, \\
& H^{i}\left(\mathcal{P}_{S}^{\bullet}(n)\right) \longrightarrow\left(g r_{n}^{\gamma} K_{2 n-i}(\mathbb{C})_{\mathbb{Q}} \otimes \varepsilon(n)\right)^{+} .
\end{aligned}
$$

The conjecture follows from the motivic interpretation of the Dehn complexes and standard conjectures on the mixed Tate motives; see Section 5 . For a conjecture relating the cohomology of the euclidean Dehn complexes to the absolute Kahler differentials $\Omega_{\mathbb{R} / \mathbb{Q}}^{i}$ see s. 6.3 . 
Conjecture 1.9. The homomorphisms in 1.8 are isomorphisms.

If $n=2$, this is true thanks to the above-mentioned results of Dupont and Sah. This conjecture, unlike the previous one, goes beyond the standard conjectures.

Let $K_{n}^{M}(F)$ be the Milnor $K$-group of a field $F$. By definition it is a quotient of $\otimes^{n} F^{*}$ by the subgroup generated by the Steinberg elements $y_{1} \otimes \ldots \otimes(1-x) \otimes x \otimes$ $\cdots \otimes y_{n}$. It was proved by Suslin that $K_{n}^{M}(F)$ is isomorphic to $g r_{n}^{\gamma} K_{n}(F)$ modulo $(n-1)$ ! torsion. The following result supports Conjecture 1.8.

Proposition 1.10. There are canonical surjective homomorphisms

$$
H^{n}\left(\mathcal{P}_{\mathcal{H}}^{\bullet}(n)\right) \longrightarrow\left(K_{n}^{M}(\mathbb{C}) \otimes \varepsilon(n)\right)^{-}, \quad H^{n}\left(\mathcal{P}_{S}^{\bullet}(n)\right) \longrightarrow\left(K_{n}^{M}(\mathbb{C}) \otimes \varepsilon(n)\right)^{+} .
$$

7. The structure of the paper. Theorem 1.1 is proved in Section 2. The essential part of the proof of Theorem 1.5 is Theorem 3.11 which is proved in Section 3; for the last step of the proof of Theorem 1.5 see s. 5.2. Proposition 1.10 is proved in Section 6.1.

To prove Theorem 1.6 we need to study periods of Hodge-Tate structures and construct the abelian category of mixed Tate motives over a number field. Sections 4 and 5 are devoted to these topics, which are of independent interest.

In Section 4 we study the periods of mixed Hodge-Tate structures. In general a period of a variation of mixed Hodge structures over a complex manifold $S$ is a multivalued analytic function on $S$. It has a single-valued counterpart: the real period. (In fact there are two different kinds of real periods.) We introduce in Section 4 the big period of a Hodge-Tate structure, which is a well-defined section of $\mathcal{O}_{S} \otimes_{\mathbb{Q}} \mathcal{O}_{S}^{*}(n-2)$. The real period is obtained from it by the natural projection $(\operatorname{Im}(x+i y):=i y)$ :

$$
\mathbb{C} \otimes \mathbb{Q} \mathbb{C}^{*}(n-2) \longrightarrow \mathbb{R}(n-1), \quad a \otimes b \otimes(2 \pi i)^{n-2} \longmapsto(2 \pi i)^{n-2} \operatorname{Im} a \cdot \log |b| .
$$

We show that the volume of a hyperbolic geodesic simplex is indeed the real period of the corresponding Hodge-Tate structure. This is a more subtle fact than the algebraic geometric interpretation of the volume by integral (5), which shows only that the volume is some kind of period. The proof uses the big period map and some peculiar properties of the coproduct of the Hodge-Tate structure corresponding to a geodesic simplex. This is needed to prove commutativity of the diagrams in Theorem 1.6. The existence of the horizontal arrows in these diagrams is established in Section 5.

In s. 5.3-5.4 some known to the experts results about triangulated categories and their abelian hearts are proved. Together with Voevodsky's results they insure the existence of the category of mixed Tate motives over a number field with all the expected properties. This result should have quite a lot of different applications. We present one of them in Section 5, deducing Theorem 1.6 from Theorem 1.5.

In Section 3 we construct a commutative graded Hopf algebra $S_{\bullet}(\mathbb{C})$ which is the complex analog of the classical scissors congruence groups. In fact the construction works without any changes for any algebraically closed field $F$. The dual of the corresponding Hopf algebra $S_{\bullet}(F)$ is the universal enveloping algebra of a certain negatively graded (pro)-Lie algebra. I think that the category of finite-dimensional graded modules over this Lie algebra is equivalent to the category of mixed Tate motives over $F$. In other words this Lie algebra is conjectured to be the Lie algebra of the unipotent part of the maximal Tate quotient of the motivic Galois group of $\operatorname{Spec}(F)$ (compare with [BGSV]). In particular the cohomology of $S_{\bullet}(F)$ should 
coincide with the appropriate pieces of the algebraic K-theory of $F$; see Conjecture 5.1 .

\section{Hyperbolic VOlumes AND Borel's REgulator}

1. Homological interpretation of the volume of a compact hyperbolic manifold. Any oriented hyperbolic manifold $M^{n}, \operatorname{dim} M^{n}=n$, can be represented as a quotient $M^{n}=\Gamma \backslash \mathcal{H}^{n}$ where $\Gamma$ is a torsion free subgroup of $S O(n, 1)$.

Let us denote by $B G$ the classifying space of a group $G$. Set $G^{n}:=\underbrace{G \times \cdots \times G}_{n \text { times }}$. There is Milnor's simplicial model for $B G: B G_{\bullet}=E G_{\bullet} / G$ where

$$
E G_{\bullet}: G \leftleftarrows G^{2} \leftleftarrows G^{3} \overleftarrow{\leftarrow} \ldots
$$

The inclusion $j: \Gamma \hookrightarrow S O(n, 1)$ induces a map

$$
j_{*}: B \Gamma \rightarrow B S O(n, 1)^{\delta} .
$$

(Here $G^{\delta}$ is a Lie group considered as a discrete group.) Notice that $\Gamma \backslash \mathcal{H}^{n}=B \Gamma$.

Suppose that $M^{n}$ is compact. Then $H_{n}\left(M^{n}\right)=\mathbb{Z}$. Let us denote by $b_{n}$ the generator of $H_{n}\left(M^{n}\right)$. Then

$$
j_{*}\left(b_{n}\right) \in H_{n}\left(B S O(n, 1)^{\delta}\right)=H_{n}\left(S O(n, 1)^{\delta}\right) .
$$

Further, let $G$ be a Lie group and $C^{n}(G)$ the space of continuous functions on $G^{n}$. There is a differential

$$
d: C^{n}(G) \rightarrow C^{n+1}(G), \quad d f\left(g_{0}, \ldots, g_{n}\right)=\sum_{i=0}^{n}(-1)^{i} f\left(g_{0}, \ldots, \hat{g}_{i}, \ldots, g_{n}\right) .
$$

Let $C^{n}(G)^{G}$ be the subspace of functions invariant under the left diagonal action of $G$ on $G^{n}$. Then by definition

$$
H_{c}^{*}(G, R):=H^{*+1}\left(\ldots \stackrel{d}{\longrightarrow} C^{n}(G)^{G} \stackrel{d}{\longrightarrow} C^{n+1}(G)^{G} \stackrel{d}{\longrightarrow} \ldots\right)
$$

is the continuous cohomology of the Lie group $G$.

Let us denote by $I\left(g_{0} x, \ldots, g_{n} x\right)$ the geodesic simplex in the hyperbolic space $\mathcal{H}^{n}$ with vertices at points $g_{0} x, \ldots, g_{n} x$, where $g_{i} \in S O(n, 1)$ and $x$ is a given point in $\mathcal{H}^{n}$. Then

$$
\operatorname{vol} I\left(g_{0} x, \ldots, g_{n} x\right)
$$

is a continuous $n$-cocycle of $S O(n, 1)$ because it is invariant under the left diagonal action of $S O(n, 1)$ and satisfies the cocycle condition

$$
\sum_{i=0}^{n+1}(-1)^{i} \operatorname{vol} I\left(g_{0} x, \ldots, \widehat{g_{i} x}, \ldots, g_{n+1} x\right)=0 .
$$

Let

$$
v_{n} \in H_{c}^{n}(S O(n, 1), R) \subset H^{n}\left(S O(n, 1)^{\delta}, R\right)
$$

be the cohomology class of this cocycle.

Theorem 2.1. Let $M^{n}$ be a compact hyperbolic manifold. Then

$$
\operatorname{vol}\left(M^{n}\right)=\left\langle v_{n}, j_{*}\left(b_{n}\right)\right\rangle .
$$


Proof. There is a triangulation $M^{n}=\bigcup_{k} I_{k}$ of $M^{n}$ by geodesic simplices $I_{k}$. To obtain it choose $N$ generic points $y_{1}, \ldots, y_{N}$ in $M^{n}$ and consider the corresponding Dirichlet domains

$$
\mathcal{D}\left(y_{j}\right):=\left\{x \in M^{n} \mid \rho\left(x, y_{\alpha}\right) \leq \rho\left(x, y_{\beta}\right) \text { for any } \beta \neq \alpha\right\} .
$$

Then the dual triangulation is the desired one. (The vertices of the dual simplices are the points $y_{\alpha}$; two vertices $y_{\alpha_{1}}$ and $y_{\alpha_{2}}$ are connected by the edge if domains $\mathcal{D}\left(y_{\alpha_{1}}\right)$ and $\mathcal{D}\left(y_{\alpha_{2}}\right)$ have a common codimension-1 face and so on.)

Let $\pi: \mathcal{H}^{n} \rightarrow \Gamma \backslash \mathcal{H}^{n}$. The group $\Gamma$ acts on $\mathcal{H}^{n}$ freely. Therefore $\pi^{-1}\left(y_{\alpha}\right)$ is a principal homogeneous space of $\Gamma$. Let us choose elements $g_{\alpha} \in S O(n, 1)$ such that $g_{\alpha} x \in \pi^{-1}\left(y_{\alpha}\right)$ (and so $\left.\pi^{-1}\left(y_{\alpha}\right)=\Gamma \cdot g_{\alpha} x\right)$. Let $I\left(g_{0}^{(k)} x, \ldots, g_{n}^{(k)} x\right)$ be a geodesic simplex in $\mathcal{H}^{n}$ that projects onto $I_{k}$. One can choose elements $g_{i}^{(k)}$ in such a way that $g_{i}^{(k)} \in \Gamma \cdot g_{\alpha}$ for some $\alpha$. Let us do this and consider the following $n$-chain in $B S O(n, 1)^{\delta}$ :

$$
\sum_{k}\left(g_{0}^{(k)}, \ldots, g_{n}^{(k)}\right)
$$

Lemma 2.2. a) The boundary of this $n$-chain is zero.

b) Its homology class in $H_{n}\left(B S O(n, 1)^{\delta}\right)$ coincides with $j_{*}\left(b_{n}\right)$.

Proof. Let $I\left(h_{0}^{(1)} x, \ldots, h_{n-1}^{(1)} x\right)$ and $I\left(h_{0}^{(2)} x, \ldots, h_{n-1}^{(2)} x\right)$ be codimension-1 faces of geodesic simplices $I\left(g_{0}^{(k)} x, \ldots, g_{n}^{(k)} x\right)$ that project to the same face in $\Gamma \backslash \mathcal{H}^{n}$. Then (thanks to the special choice of elements $g_{i}^{(k)}$ ) there is an element $\gamma \in \Gamma$ such that $\gamma \cdot h_{i}^{(1)}=h_{i}^{(2)}$ for all $i$. Further, it is clear that these faces will appear with opposite signs. Statement a) is proved. The proof of statement b) follows from the definitions.

The value of the cocycle (11) on the cycle (14) is equal to $\operatorname{vol}\left(\Gamma \backslash \mathcal{H}^{n}\right)$ just by definition. So Theorem 2.1 follows immediately from Lemma 2.2.

2. The strategy for noncompact hyperbolic manifolds. Now let $M^{n}=$ $\Gamma \backslash \mathcal{H}^{n}$ be a noncompact hyperbolic manifold with finite volume. We would like to prove an analog of Theorem 2.1. The first problem is that $H_{n}\left(M^{n}\right)=0$. Let $H$ be a subgroup of a group $G$. Set

$$
H_{*}(G, H ; \mathbb{Q}):=H_{*}\left(\operatorname{Cone}\left(B H_{\bullet} \rightarrow B G_{\bullet}\right), \mathbb{Q}\right) .
$$

We will show that $M^{n}$ does produce an element

$$
\tilde{c}\left(M_{n}\right) \in H_{n}\left(S O(n, 1)^{\delta}, T_{n}(s)^{\delta} ; \mathbb{Q}\right)
$$

where $T_{n}(s)$ is a subgroup of $S O(n, 1)$ consisting of transformations preserving $s \in \partial \mathcal{H}^{n}$ and acting as translations on horospheres based at $s$. (If $s$ is the point at infinity in the upper half-space realization of $\mathcal{H}^{n}$, then $T_{n}(s)$ is the group of translations in the hyperplane $x_{n}=c$ where $x_{n}$ is the vertical coordinate.)

Further, let $H$ be a Lie subgroup of a Lie group $G$. The inclusion $i: H \hookrightarrow G$ induces a homomorphism of complexes $C^{*}(G)^{G} \longrightarrow C^{*}(H)^{H}$. Set

$$
H_{c}^{*}(G, H ; \mathbb{R}):=H^{*} \operatorname{Cone}\left(C^{*}(G)^{G} \stackrel{i^{*}}{\longrightarrow} C^{*}(H)^{H}\right)[-1] .
$$

By definition an $n$-cocycle in $\operatorname{Cone}\left(C^{*}(G)^{G} \stackrel{i^{*}}{\longrightarrow} C^{*}(H)^{H}\right)[-1]$ is a pair $(f, h)$ where $f$ is a continuous $n$-cocycle of $G, h$ is an $(n-1)$-cochain on $H$ and $i^{*} f=d h$. In 
other words, cocycles in Cone $\left(i^{*}\right)$ are just those cocycles of $G$ whose restriction to $H$ is cohomologous to zero.

Let us denote by $E_{n}(s)$ the stabilizer in $S O(n, 1)$ of a point $s \in \partial \mathcal{H}^{n}$. Choose a point $x \in \mathcal{H}^{n}$. Then for $h_{i} \in E_{n}(s)$

$$
\bar{v}_{n}(x ; s):=\left(\operatorname{vol} I\left(g_{0} x, \ldots, g_{n} x\right), \operatorname{vol} I\left(s, h_{0} x, \ldots, h_{n-1} x\right)\right)
$$

is a cocycle representing a certain cohomology class

$$
\bar{v}_{n} \in H_{c}^{n}\left(S O(n, 1), E_{n}(s)\right) .
$$

Indeed, $\operatorname{vol} I\left(h_{0} x, \ldots, h_{n} x\right)=\sum_{i=0}^{n}(-1)^{i} \operatorname{vol} I\left(s, h_{0} x, \ldots, \widehat{h_{i} x}, \ldots, h_{n} x\right)$. In particular since $T_{n}(s)$ is a subgroup of $E_{n}(s)$, this cocycle provides a cocycle $\tilde{v}_{n}(x)$ representing a cohomology class

$$
\tilde{v}_{n} \in H_{c}^{n}\left(S O(n, 1), T_{n}(s)\right) .
$$

We will prove that $\operatorname{vol}\left(M^{n}\right)=\left\langle\tilde{v}_{n}, \tilde{c}\left(M^{n}\right)\right\rangle$.

To produce the class $\tilde{c}_{n}\left(M^{n}\right)$ we have to recall some basic facts about noncompact hyperbolic manifolds (see $\S 5$ of Thurston's book [Th]).

3. A decomposition of hyperbolic manifolds. Let $x \in \mathcal{H}^{n}$. Denote by $\Gamma_{\varepsilon}(x)$ the subgroup generated by all elements of $\Gamma$ which move $x$ to a distance $\leq \varepsilon$.

Theorem 2.3 (Kazhdan-Margoulis). There is an $\varepsilon>0$ such that for every discrete group $\Gamma$ of isometries of $\mathcal{H}^{n}$ and for every $x \in \mathcal{H}^{n}, \Gamma_{\varepsilon}(x)$ has an abelian subgroup of finite index.

Let $s \in \partial \mathcal{H}^{n}$. A part of $\mathcal{H}^{n}$ located inside of a horosphere centered at $s$ is called a horoball. In the upper half-space realization of an $\mathcal{H}^{n}$ horoball centered at infinity is defined as $\left\{x_{n} \geq c\right\}$. Let $\Delta_{n}(s)$ be a discrete subgroup of $E_{n}(s)$ that has a subgroup of finite index isomorphic to $\mathbb{Z}^{n-1}$ and acting by translation on horospheres centered at $s$. Suppose also that $\Delta_{n}(s)$ acts freely in $\mathcal{H}^{n}$. Then the quotient of a horoball centered at $s$ is called the cusp.

Denote by $\ell(v)$ the length of the shortest geodesic loop based at $v$.

Theorem 2.4 (Decomposition Theorem). Let $M^{n}$ be an orientable hyperbolic manifold of finite volume. Then there is a $\delta>0$ such that $\ell^{-1}[0, \delta] \subset M^{n}$ consists of finitely many components and each of these components is isometric to a cusp.

This theorem follows from Theorem 2.3.

Recall that $M^{n}=\Gamma \backslash \mathcal{H}^{n}$. Let us denote by $C(\Gamma)$ the subset of $\partial \mathcal{H}^{n}$ consisting of the points $c$ such that

a) the isotropy subgroup of $c$ in $\Gamma$ is nontrivial,

b) the isotropy subgroup of any geodesic ending at $c$ in $\Gamma$ is trivial.

Remark. A nontrivial element $\gamma$ of $\Gamma$ preserving a point $c \in \partial \mathcal{H}^{n}$ and a geodesic ending at $c$ cannot stabilize points on this geodesic because by the assumption each point of $\mathcal{H}^{n}$ has trivial stabilizer in $\Gamma$. So the element $\gamma$ moves the geodesic along itself. Therefore the point $c$ does not correspond to a cusp of $\Gamma \backslash \mathcal{H}^{n}$. It follows from the Decomposition Theorem that $C(\Gamma)$ consists of a finite number of $\Gamma$-orbits. (Each of them corresponds to a cusp of $\Gamma \backslash \mathcal{H}^{n}$.) Let us choose $\left\{c_{i}\right\} \in C(\Gamma)$ such 
that

$$
C(\Gamma)=\bigcup_{i} \Gamma c_{i}
$$

The manifold $M^{n}$ is compact if and only if $C(\Gamma)=\emptyset$.

4. A triangulation of a noncompact hyperbolic manifold by ideal geodesic simplices. We would like to define a Dirichlet decomposition of $\mathcal{H}^{n}$ corresponding to the set $C(\Gamma) \in \partial \mathcal{H}^{n}$, and then take the dual triangulation. The problem is that the distance $\rho(x, c)$ from a point $x \in \mathcal{H}^{n}$ to $c \in C(\Gamma)$ is infinite. To define a regularized distance $\tilde{\rho}(x, c)$ let us choose a horosphere $h(c)$ for each $c \in C(\Gamma)$ in such a way that $h(\gamma \cdot c)=\gamma \cdot h(c)$ for any $\gamma \in \Gamma$. Then by definition $\tilde{\rho}(x, c)$ is the distance from $x$ to the horosphere $h(c)$. It is negative if $x$ is inside of $h(c)$. Now the Dirichlet domains are defined as usual:

$$
\mathcal{D}(c):=\left\{x \in \mathcal{H}^{n} \mid \tilde{\rho}(x, c) \leq \tilde{\rho}\left(x, c^{\prime}\right) \text { for any } c^{\prime} \in C(\Gamma), c^{\prime} \neq c\right\} .
$$

They are polyhedrons with a finite number of faces.

For a generic choice of horospheres $h\left(c_{i}\right)$ the dual polyhedrons are simplices with vertices at points of $C(\Gamma)$. This triangulation is $\Gamma$-invariant.

\section{Homological interpretation of the hyperbolic volumes.}

Theorem 2.5. Any hyperbolic manifold of finite volume $M^{n}$ defines a class

$$
\tilde{c}\left(M^{n}\right) \in H_{n}\left(S O(n, 1)^{\delta}, T_{n}(v)^{\delta} ; \mathbb{Q}\right)
$$

such that

$$
\left\langle\tilde{v}_{n}, \tilde{c}\left(M^{n}\right)\right\rangle=\operatorname{vol}\left(M^{n}\right) .
$$

Proof. Let $\widetilde{\partial \mathcal{H}^{n}}$ be the set of all pairs $(v, \ell)$ where $v \in \partial \mathcal{H}^{n}$ and $\ell$ is a geodesic ending at $v$.

Let $\widetilde{C(\Gamma)} \subset \widetilde{\partial \mathcal{H}^{n}}$ be the set of all pairs $(c, \ell)$ where $c \in C(\Gamma)$ and $\ell$ is an edge of one of the geodesic simplices from the constructed triangulation of $\mathcal{H}^{n}$. Notice that the group $\Gamma$ acts freely on $\overparen{C(\Gamma)}$. Choose geodesics $\ell_{i j}$ such that $\left(c_{i}, \ell_{i j}\right) \subset \widetilde{C(\Gamma)}$ and

$$
\widetilde{C(\Gamma)}=\bigcup_{i, j} \Gamma \cdot\left(c_{i}, \ell_{i j}\right) .
$$

Take a point $\left(v_{0}, \ell_{0}\right) \in \widetilde{\partial \mathcal{H}^{n}}$ such that $v_{0} \notin C(\Gamma)$. There exist elements $g_{i j} \in$ $S O(n, 1)$ with properties

$$
\text { a) }\left(c_{i}, \ell_{i j}\right)=g_{i j} \cdot\left(v_{0}, \ell_{0}\right), \quad \text { b) } g_{i j}^{-1} g_{i k} \in T_{n}\left(v_{0}\right) \text {. }
$$

(Recall that $T_{n}\left(v_{0}\right)$ is the subgroup of translations.) Therefore any $(c, \ell) \in \widetilde{C(\Gamma)}$ can be written as

$$
\begin{gathered}
(c, \ell)=g_{(c, \ell)} \cdot\left(v_{0}, \ell_{0}\right), \\
g_{(c, \ell)}:=\gamma_{(c, \ell)} \cdot g_{i j},
\end{gathered}
$$

where $\gamma_{(c, \ell)} \in \Gamma$ is defined from the condition $(c, \ell)=\gamma_{(c, \ell)} \cdot\left(c_{i}, \ell_{i j}\right)$.

Let us choose a representation $S_{\alpha}$ in each $\Gamma$-equivalence class of simplices from the constructed triangulation of $\mathcal{H}^{n}$. This is a finite set $\left\{S_{\alpha}\right\}$ and $\left\{\pi\left(S_{\alpha}\right)\right\}$ is a triangulation of $\Gamma \backslash \mathcal{H}^{n}$. (Recall that $\pi: \mathcal{H}^{n} \rightarrow \Gamma \backslash \mathcal{H}^{n}$.) 
Let $F\left(\left\{S_{\alpha}\right\}\right) \subset \widetilde{C(\Gamma)}$ be the set of all pairs $(v, \ell)$ where $v$ is a vertex of a simplex $S_{\alpha}$ and $\ell$ is its edge. Each simplex $S_{\alpha}$ defines $n(n+1)$ elements of the set $F\left(\left\{S_{\alpha}\right\}\right)$. They correspond to vertices of the "truncated simplex" $\tilde{S}_{\alpha}$. Let us subdivide polyhedrons $\tilde{S}_{\alpha}$ on simplices $\tilde{S}_{\alpha, \beta}$. Denote by $f_{\alpha, \beta}^{0}, \ldots, f_{\alpha, \beta}^{n}$ the vertices of $\tilde{S}_{\alpha, \beta}$. According to (20) there are uniquely defined elements $g_{\alpha, \beta}^{i} \in S O(n, 1)$ such that $f_{\alpha \beta}^{i}=g_{\alpha \beta}^{i} \cdot\left(v_{0}, \ell_{0}\right) \quad(0 \leq i \leq n)$. Set

$$
\tilde{c}^{\prime}\left(M^{n}\right):=\sum_{\alpha, \beta}\left(g_{\alpha, \beta}^{0}, \ldots, g_{\alpha, \beta}^{n}\right) \in B S O(n, 1)_{n} .
$$

The boundary of chain (22) is computed as follows. Each vertex $v_{a}\left(S_{\alpha}\right)$ of simplex $S_{\alpha}$ gives us $n$ elements

$$
\left(v_{a}\left(S_{\alpha}\right), \ell_{a}^{1}\left(S_{\alpha}\right)\right), \ldots,\left(v_{a}\left(S_{\alpha}\right), \ell_{a}^{n}\left(S_{\alpha}\right)\right)
$$

in $F\left(\left\{S_{\alpha}\right\}\right)$. So there are $h_{a}^{b} \in S O(n, 1)$, chosen according to (20)-(21), such that

$$
\left(v_{a}\left(S_{\alpha}\right), \ell_{a}^{b}\left(S_{\alpha}\right)\right)=h_{a}^{b}\left(S_{\alpha}\right) \cdot\left(v_{0}, \ell_{0}\right)
$$

$\left(\left\{h_{a}^{b}\left(S_{\alpha}\right)\right\}=\left\{g_{\alpha, \beta}^{i}\right\}\right.$ of course). So each vertex $v_{a}\left(S_{\alpha}\right)$ produces a chain

$$
\left(h_{a}^{1}\left(S_{\alpha}\right), \ldots, h_{a}^{n}\left(S_{\alpha}\right)\right) \in B S O(n, 1)_{n-1} .
$$

It follows from the definitions that

$$
\partial \tilde{c}_{n}^{\prime}\left(M^{n}\right)=\sum_{v_{a}\left(S_{\alpha}\right)}\left(h_{a}^{1}\left(S_{\alpha}\right), \ldots, h_{a}^{n}\left(S_{\alpha}\right)\right)
$$

where summation is over all vertices of simplices $S_{\alpha}$.

Lemma 2.6. For every $c_{i}$ (see (17))

$$
\sum_{v_{a}\left(S_{\alpha}\right) \in\left\{\Gamma \cdot c_{i}\right\}}\left(h_{a}^{1}\left(S_{\alpha}\right), \ldots, h_{a}^{n}\left(S_{\alpha}\right)\right) \in B S O(n, 1)_{n-1}
$$

is a cycle.

Proof. Clear.

Lemma 2.7. The cycle (23) is the image of a cycle $\tilde{c}_{(i)}^{\prime \prime}\left(M^{n}\right)$ in $B G^{(i)}\left(v_{0}\right)_{(n-1)}$ where $G^{(i)}\left(v_{0}\right)$ is a semidirect product of a finite group and the group of translations $T\left(v_{0}\right)$.

Proof. Look at definitions (19). Each element $h_{a}^{b}\left(S_{\alpha}\right)$ is equal to

$$
\gamma \cdot g_{i k} \cdot\left(v_{0}, \ell_{0}\right)=\gamma \cdot g_{i j} \cdot t \cdot\left(v_{0}, \ell_{0}\right)=g_{i j} \cdot\left(g_{i j}^{-1} \cdot \gamma \cdot g_{i j}\right) \cdot t \cdot\left(v_{0}, \ell_{0}\right)
$$

where $\gamma \in \Delta_{n}\left(c_{i}\right)$ (the stabilizer of $c_{i}$ in $\Gamma$ ) and $t \in T\left(v_{0}\right)$. Notice that $g_{i j}^{-1} T\left(c_{i}\right) g_{i j}=$ $T\left(v_{0}\right)$ and $\Delta_{n}\left(c_{i}\right)$ is a semidirect product of a finite group and a subgroup in $T\left(c_{i}\right)$. Lemma 2.7 is proved.

It follows from Lemma 2.6 and Lemma 2.7 that

$$
c_{E}\left(M^{n}\right):=\left(\sum_{i} \tilde{c}_{(i)}^{\prime \prime}\left(M^{n}\right), \tilde{c}^{\prime}\left(M^{n}\right)\right)
$$

is a cycle in

$$
\text { Cone }\left(B E_{n}\left(v_{0}\right) \bullet \rightarrow B S O(n, 1) \bullet\right) .
$$

Proposition 2.8. $\left\langle\bar{v}_{n}, c_{E}\left(M^{n}\right)\right\rangle=\operatorname{vol}\left(M^{n}\right)$. 
Proof. Recall that the cocycle $\bar{v}_{n}\left(x ; v_{0}\right)$ (see $\left.(16)\right)$ represents the cohomology class $\bar{v}_{n} \in H_{c}^{n}\left(S O(n, 1), E_{n}\left(v_{0}\right)\right)$. The cocycles $\bar{v}_{n}\left(x ; v_{0}\right)$ for different points $x \in \mathcal{H}^{n}$ are (canonically) cohomologous. Now let us move point $x$ in (16) to the boundary point $v_{0}$. Then the limit of value of the component $\operatorname{vol}\left(I\left(g_{0} x, \ldots, g_{n} x\right)\right)$ on the chain $\tilde{c}^{\prime}\left(M^{n}\right)$ (see (22)) exists and is equal to $\sum_{\alpha} \operatorname{vol}\left(S_{\alpha}\right)=\operatorname{vol}\left(M^{n}\right)$. On the other hand, the limit of the second component $\operatorname{vol} I\left(v_{0}, h_{0} x, \ldots, h_{n-1} x\right)$ of cocycle $\bar{v}_{n}\left(x ; v_{0}\right)$ on the chain $\sum_{i} c_{(i)}^{\prime \prime}\left(M^{n}\right)$ is zero. Proposition 2.8 is proved.

To prove Theorem 2.5 it remains to show that there is a cycle $c_{T}\left(M^{n}\right)$ in

$$
\text { Cone }\left(B T_{n}\left(v_{0}\right) \bullet \rightarrow B S O(n, 1) \bullet\right)
$$

homologous to $N \cdot c_{E}\left(M^{n}\right)$ for certain integer $N$. This is a consequence of the following

Lemma 2.9. The homomorphism

$$
j_{*}: H_{*}\left(T_{n}\left(v_{0}\right), \mathbb{Q}\right) \rightarrow H_{*}\left(G^{i}\left(v_{0}\right), \mathbb{Q}\right)
$$

induced by the inclusion $j: T_{n}\left(v_{0}\right) \hookrightarrow G^{(i)}\left(v_{0}\right)$ is a map onto.

Proof. Set $A:=G^{(i)}\left(v_{0}\right) / T_{n}\left(v_{0}\right)$. There is a Hochshild-Serre spectral sequence

$$
E_{p q}^{2}=H_{p}\left(A, H_{q}\left(T_{n}\left(v_{0}\right), \mathbb{Q}\right)\right) \Rightarrow H_{p+q}\left(G^{(i)}\left(v_{0}\right), \mathbb{Q}\right) .
$$

Further, for a finite group $A$ and an $A$-module $V, H_{i}(A, V \otimes \mathbb{Q})=0$ for $i>0$. Therefore

$$
H_{*}\left(G^{(i)}\left(v_{0}\right), \mathbb{Q}\right)=H_{*}\left(T_{n}\left(v_{0}\right), \mathbb{Q}\right)_{A} .
$$

Lemma 2.9 and hence Theorem 2.5 are proved.

6. $\tilde{c}\left(M^{2 n-1}\right)$ and $H_{2 n-1}\left(G L_{N}(\overline{\mathbb{Q}}), \mathbb{Q}\right)$. It is usefull to keep in mind the following trivial lemma.

Lemma 2.10. Let $\tilde{G} \rightarrow G$ be a surjective homomorphism of groups with finite kernel. Then it induces an isomorphism on homology with rational coefficients.

Let $N=2^{n-1}$ and $s_{+}: \operatorname{Spin}(2 n-1,1) \rightarrow G L_{N}(\mathbb{C})$ be the half-spinor representation. Set $\tilde{T}_{2 n-1}(v):=s_{+}\left(T_{2 n-1}(v)\right)$. We will produce a basis in this representation and a 1-dimensional subgroup $H \subset \operatorname{diag}\left(G L_{N}\right)$ isomorphic to $\Gamma_{m}$ and diagonal in this basis such that the following 3 conditions hold:

1. $s_{+}\left(\operatorname{Lie}\left(T_{2 n-1}(v)\right)\right)$ is an abelian Lie algebra contained in the upper triangular Lie algebra.

2. It is normalized by a subgroup $H$.

3. The eigenvalues of an element $t \in H(\mathbb{Q})=\mathbb{Q}^{*}$ acting on $s_{+}\left(\operatorname{Lie}\left(T_{2 n-1}(v)\right)\right)$ by conjugation are equal to $t$.

For this let us recall the construction of the half-spinor representation. The spinor representation of the complex Lie algebra $o\left(V_{2 n}\right)$, where $V_{2 n}$ is a $2 n$-dimensional complex vector space, can be described as follows. Let us choose a decomposition $V_{2 n}=U \oplus W$ where $U$ and $W$ are (maximal) isotropic planes. Then $o\left(V_{2 n}\right)$ is isomorphic to a Lie subalgebra of the Lie algebra of all superdifferential operators of order $\leq 2$ acting on the vector space $\Lambda^{*} W$ (which is the space of regular functions on the odd variety $W)$. To be precise, $o\left(V_{2 n}\right)$ is the Lie subalgebra of all superdifferential operators of total degree 0 or 2 .

Notice that

$$
\Lambda^{*} W=S^{-} \oplus S^{+}
$$


where $S^{-}=\Lambda^{\text {odd }} W, S^{+}=\Lambda^{\text {even }} W$. Each subspace is preserved by the action of $o\left(V_{2 n}\right)$. The corresponding representations are the half-spinor representations $s_{+}$ and $s_{-}$.

Let us describe the spinor representation in coordinates. Choose a basis $e_{1}, \ldots, e_{n}$; $f_{1}, \ldots, f_{n}$ in $V_{2 n}$ such that $W=\left\langle e_{1}, \ldots, e_{n}\right\rangle ; U=\left\langle f_{1}, \ldots, f_{n}\right\rangle$ and $\left(e_{i}, f_{j}\right)=\delta_{i j}$. Then an element $X \in o(2 n)$ looks in this basis as follows:

$$
\left(\begin{array}{cc}
A & B \\
C & -A^{t}
\end{array}\right)
$$

where $B^{t}=-B$ and $C^{t}=-C$. One has

$$
s: X \longmapsto-\frac{1}{2} \operatorname{tr} A+\sum_{1 \leq i, j \leq n} a_{i j} \xi_{i} \partial_{\xi_{j}}+\sum_{1<i<j<n}\left(b_{i j} \xi_{i} \wedge \xi_{j}+c_{i j} \partial_{\xi_{i}} \wedge \partial_{\xi_{j}}\right) .
$$

Let $\mathcal{N} \subset s(o(2 n))$ be the Lie subalgebra acting in the half-spinor representation by the following operators:

$$
\sum_{1<j<n} a_{1 j} \xi_{1} \partial_{\xi_{j}}+\sum_{1<j<n} b_{1 j} \xi_{1} \wedge \xi_{j}
$$

We will denote by $N$ the corresponding subgroup of the group $G L_{N}$. The group $\tilde{T}_{2 n-1}(v)$ is conjugated to a subgroup of $N$.

Let us embed the group $\mathbb{G}_{m}$ to $G L_{N}$ as follows: $t \in \mathbb{G}_{m}$ multiplies a vector $\xi_{i_{1}} \wedge \ldots \wedge \xi_{i_{k}}$ where $i_{1}<\ldots<i_{k}$ by $t$ if $i_{1}=1$ and by 1 otherwise. This is the subgroup $H$. Let $P$ be the semidirect product of the groups $H$ and $N$.

Let us denote by $\hat{c}\left(M^{2 n-1}\right)$ the image of $\tilde{c}\left(M^{2 n-1}\right)$ under the composition of natural homomorphisms

$$
\begin{gathered}
H_{2 n-1}\left(S O(2 n-1,1), T_{2 n-1}(v) ; \mathbb{Q}\right) \stackrel{s_{+*}}{\longrightarrow} H_{2 n-1}\left(G L_{N}(\mathbb{C}), \tilde{T}_{2 n-1}(v) ; \mathbb{Q}\right) \\
\longrightarrow H_{2 n-1}\left(G L_{N}(\mathbb{C}), P(\mathbb{C}) ; \mathbb{Q}\right) .
\end{gathered}
$$

The second arrow is provided by a conjugation in $G L_{N}(\overline{\mathbb{Q}})$. Let $P(\overline{\mathbb{Q}})$ be the group of $\overline{\mathbb{Q}}$-points of $P$.

Lemma 2.11. a) Let $M^{2 n-1}$ be a compact hyperbolic manifold. Then (see (10))

$$
j_{*}\left(b_{2 n-1}\right) \in H_{n}(S O(2 n-1,1)(\overline{\mathbb{Q}}), \mathbb{Z}) .
$$

b) Let $M^{2 n-1}$ be a noncompact hyperbolic manifold. Then

$$
\hat{c}\left(M^{2 n-1}\right) \in H_{2 n-1}\left(G L_{N}(\overline{\mathbb{Q}}), P(\overline{\mathbb{Q}}) ; \mathbb{Q}\right) .
$$

Proof. According to Weil's rigidity (see 6.6, 6.7, 7.13 in [Ra]), the subgroup $\Gamma \in$ $S O(2 n-1,1)$ is conjugate to a subgroup whose entries are algebraic numbers. In particular the cusps of a hyperbolic manifold are defined over $\overline{\mathbb{Q}}$.

There is the usual exact sequence of cone:

$$
\ldots \rightarrow H_{2 n-1}(P(\overline{\mathbb{Q}}) ; \mathbb{Q}) \longrightarrow H_{2 n-1}\left(G L_{N}(\overline{\mathbb{Q}}) ; \mathbb{Q}\right) \stackrel{e_{*}}{\longrightarrow} H_{2 n-1}\left(G L_{N}(\overline{\mathbb{Q}}), P(\overline{\mathbb{Q}}) ; \mathbb{Q}\right)
$$

$$
\longrightarrow H_{2 n-2}(P(\overline{\mathbb{Q}}) ; \mathbb{Q}) \longrightarrow H_{2 n-2}\left(G L_{N}(\overline{\mathbb{Q}}) ; \mathbb{Q}\right) .
$$

Theorem 2.12. There exists an element $c\left(M^{2 n-1}\right) \in H_{2 n-1}\left(G L_{N}(\overline{\mathbb{Q}}) ; \mathbb{Q}\right)$ such that $\hat{c}\left(M^{2 n-1}\right)=e_{*} c\left(M^{2 n-1}\right)$.

Proof. It follows from

Theorem 2.13. $i_{*}: H_{*}(P(\overline{\mathbb{Q}}) ; \mathbb{Q}) \rightarrow H_{*}\left(G L_{N}(\overline{\mathbb{Q}}) ; \mathbb{Q}\right)$ is injective. 
Proof. Recall that $P=H \cdot N$ where $H$ is the diagonal part of $P$ and $N$ is the unipotent one. Notice that $H(\overline{\mathbb{Q}})=\overline{\mathbb{Q}}^{*}$.

Lemma 2.14. The natural map $H_{*}(H(\overline{\mathbb{Q}}) ; \mathbb{Q}) \rightarrow H_{*}(P(\overline{\mathbb{Q}}) ; Q)$ is an isomorphism.

Proof. There is the Serre-Hochshild spectral sequence

$$
H_{p}\left(H, H_{q}(N, \mathbb{Q})\right) \Rightarrow H_{p+q}(P ; \mathbb{Q}) .
$$

For a number field $F$ the group $N(F)$ is isomorphic to the additive group of a finite-dimensional $\mathbb{Q}$-vector space $V(F)$. So $H_{q}(N(F), \mathbb{Q}) \simeq \bigwedge_{z} z \mathbb{Q}^{q} V(F)$. Further, any integer $n \in \mathbb{Q}^{*}=H(\mathbb{Q})$ acts on $H_{q}(N, \mathbb{Q})$ by multiplication on $n^{q}$. Therefore $H_{*}\left(H(\overline{\mathbb{Q}}), H_{q}(N(\overline{\mathbb{Q}}) ; \mathbb{Q})\right)$ is annihilated by multiplication on $n^{q}-1$.

This is a particular case of the following general fact (Proposition 5.4 in [ML]): let $G$ be a group and $V$ a $G$-module. Then the action of any element $g \in G$ on $G$ by conjugation and on $V$ via the $G$-module structure induces the identity map on $H_{*}(G, V)$. Lemma 2.14 is proved.

To complete the proof of Theorem 2.13 consider the homomorphism det : $G L_{N}(\overline{\mathbb{Q}}) \longrightarrow \overline{\mathbb{Q}}^{*}$. Notice that $\overline{\mathbb{Q}}^{*}=H(\overline{\mathbb{Q}}) \rightarrow G L_{N}(\overline{\mathbb{Q}}) \stackrel{\text { det }}{\longrightarrow} \overline{\mathbb{Q}}^{*}$ is given by $x \longmapsto x^{k}$ for a certain positive integer $k$. Therefore its composition with $H_{*}(H(\overline{\mathbb{Q}}) ; \mathbb{Q}) \longrightarrow$ $H_{*}\left(G L_{N}(\overline{\mathbb{Q}}) ; \mathbb{Q}\right) \longrightarrow H_{*}\left(\overline{\mathbb{Q}}^{*} ; \mathbb{Q}\right)$ is injective. The theorem is proved.

7. The spinor representation and the Pfaffian. The supertrace $S t r$ in the spinor representation $S=S^{+} \oplus S^{-}$is defined as follows:

$$
\operatorname{Str}:=\operatorname{Tr}\left|S_{+}-\operatorname{Tr}\right| S_{-}, \quad \text { i.e. } \quad \operatorname{Str}(A):=\operatorname{Tr} s_{+}(A)-\operatorname{Tr} s_{-}(A) .
$$

There is a remarkable invariant polynomial of degree $n$ on the Lie algebra $o(2 n)$ : the Pfaffian. Its restriction to the Cartan subalgebra $\left(t_{1}, \ldots, t_{n}\right), e_{i} \longmapsto t_{i} e_{i}, f_{i} \longmapsto$ $-t_{i} f_{i}$ is given by the formula $\operatorname{Pf}\left(t_{1}, \ldots, t_{n}\right)=t_{1} \cdot \ldots \cdot t_{n}$. For a skew-symmetric $2 n \times 2 n$ matrix $A$ one has $\operatorname{Pf}(A)^{2}=(-1)^{n} \operatorname{det}(A)$.

Proposition 2.15. The invariant polynomial $A \longmapsto(-1)^{n(n-1) / 2} n ! \times P f(A)$ on $o(2 n)$ is equal to the restriction of the invariant polynomial $A \longmapsto \operatorname{Str}\left(A^{n}\right)$ in the spinor representation.

Proof. Let us compute the restriction of the invariant polynomial $\operatorname{Str}\left(A^{n}\right)$ in the spinor representation to the Cartan subalgebra $e_{i} \longmapsto t_{i} e_{i}, f_{i} \longmapsto-t_{i} f_{i}$.

We will get

$$
\operatorname{Str}\left(\sum_{i=1}^{n} t_{i} \xi_{i} \partial_{\xi_{i}}-\frac{1}{2}\left(t_{1}+\ldots+t_{n}\right)\right)^{n}=(-1)^{n(n-1) / 2} n ! \cdot t_{1} \cdot \ldots \cdot t_{n} .
$$

Indeed, it is easy to see that the supertrace of the superdifferential operator $\xi_{i_{1}} \wedge$ $\ldots \wedge \xi_{i_{m}} \partial_{\xi_{1}} \wedge \ldots \wedge \partial_{\xi_{i_{m}}}$ is 0 if $m<n$ and $(-1)^{n(n-1) / 2} n$ ! if $m=n$.

Consider the involution $\sigma$ of the Lie algebra $o(2 n, \mathbb{C})$ which corresponds to the involution interchanging the "horns" of the Dynkin diagram, whose involutive subalgebra is $o(2 n-1, \mathbb{C})$.

Lemma 2.16. The involution $\sigma$ of the Lie algebra o $(2 n, \mathbb{C})$ interchanges the halfspinor representations.

Proof. The half-spinor representation is the fundamental representation corresponding to a vertex of a "horn". 
8. The Lobachevsky class $v_{2 n-1} \in H_{c}^{2 n-1}(S O(2 n-1,1), \mathbb{R})$ can be obtained by restriction of the Borel class in the half-spinor representation. Recall that for a Lie group $G$ with maximal compact subgroup $K$ one has the Van Est isomorphism

$$
H_{c}^{*}(G, \mathbb{R})=H^{*}(\text { LieG }, \text { LieK })
$$

(on the right we have the relative Lie algebra cohomology). On the other hand,

$$
H^{*}(\text { LieG }, \text { LieK })=H^{*}\left(\text { LieG } \otimes_{\mathbb{R}} \mathbb{C}, \text { LieK } \otimes_{\mathbb{R}} \mathbb{C}\right) .
$$

Let us suppose that $K=G^{\sigma}$ where $\sigma$ is an involution of the group $G$ (i.e. $G, K, \sigma$ is an involutive pair). Let $G_{\mathbb{C}}$ (resp. $K_{\mathbb{C}}$ ) be the complex Lie group corresponding to LieG $\otimes_{\mathbb{R}} \mathbb{C}$ (resp. Lie $K \otimes_{\mathbb{R}} \mathbb{C}$ ). Then $K_{\mathbb{C}}$ is the fixed point set of an involution $\sigma_{\mathbb{C}}$ of the complex Lie group $G_{\mathbb{C}}$. Finally, let $G_{u}$ be the maximal compact subgroup of $G_{\mathbb{C}}$. Then there is an involution $\sigma_{u}$ of $G_{u}$ such that $K=G_{u}^{\sigma_{u}}$. In this situation one has the isomorphism

$$
H_{c}^{*}(G, \mathbb{R})=H^{*}(\text { LieG }, \text { LieK })=H_{\text {top }}^{*}\left(G_{u} / K, \mathbb{R}\right)
$$

which is functorial with respect to the maps of the symmetric pairs.

For example when $G=\operatorname{Spin}(2 n-1,1)$ one has $K=\operatorname{Spin}(2 n-1), G_{u}=\operatorname{Spin}(2 n)$ and

$$
H_{c}^{*}(\operatorname{Spin}(2 n-1,1), \mathbb{R})=H_{c}^{*}(S O(2 n-1,1), \mathbb{R})=H_{\text {top }}^{*}\left(S^{2 n-1}, \mathbb{R}\right) .
$$

So there is just one (up to a scalar) nontrivial continuous cohomology class of the Lie group $S O(2 n-1,1)$. Such a class $v_{2 n-1}$ was produced in s. 2.2. We will call it the Lobachevsky class.

Similarly

$$
H_{c}^{*}\left(G L_{N}(\mathbb{C}), \mathbb{R}\right)=H_{\text {top }}^{*}\left(U(N) \times U(N) / U(N)_{\text {diag }}, \mathbb{R}\right)=\Lambda^{*}\left(b_{1}, b_{3}, \ldots, b_{2 N+1}\right)
$$

where $b_{2 i-1} \in H_{c}^{2 i-1}\left(G L_{N}(\mathbb{C}), \mathbb{R}\right)$ are the continuous cohomology classes corresponding to the primitive generators $B_{2 i-1}$ of $H_{t o p}^{*}(U(N), \mathbb{Z})$. Namely there is a canonical projection $p: U(N) \longrightarrow S^{2 N-1} \subset \mathbb{C}^{N}$ and $B_{2 N-1}:=p^{*}\left[S^{2 N-1}\right]$. Further, the restriction map $H^{2 i-1}(U(N), \mathbb{Z}) \longrightarrow H^{2 i-1}(U(i), \mathbb{Z})$ sends $B_{2 i-1}$ to the above-described generator. I will call them the Borel classes. (Borel [Bo2] used a different normalisation of these classes.)

Theorem 2.17. Let $s_{+}: \operatorname{Spin}(2 n-1,1) \rightarrow \operatorname{Aut}\left(S_{+}\right)$be the half-spinor representation. Then the restriction of the Borel class $b_{2 n-1}$ is proportional to the Lobachevsky class: $s_{+}^{*} b_{2 n-1}=c \cdot v_{2 n-1}, c \neq 0$.

Proof. We will need the following 3 symmetric pairs (Lie group, an involutive Lie subgroup)

$$
\begin{gathered}
(\operatorname{Spin}(2 n-1,1), \operatorname{Spin}(2 n-1)), \quad(\operatorname{Spin}(2 n, \mathbb{C}), \operatorname{Spin}(2 n-1, \mathbb{C})), \\
(\operatorname{Spin}(2 n), \operatorname{Spin}(2 n-1)) .
\end{gathered}
$$

We start with the half-spinor representation providing us with a morphism of symmetric pairs

$$
(\operatorname{Spin}(2 n-1,1), \operatorname{Spin}(2 n-1)) \longrightarrow\left(G L_{2^{n-1}}(\mathbb{C}), U\left(2^{n-1}\right)\right) .
$$

Then we complexify it, getting a morphism of symmetric pairs

$$
(\operatorname{Spin}(2 n, \mathbb{C}), \operatorname{Spin}(2 n-1, \mathbb{C})) \longrightarrow\left(G L_{2^{n-1}}(\mathbb{C}) \times G L_{2^{n-1}}(\mathbb{C}), G L_{2^{n-1}}(\mathbb{C})\right) .
$$


Finally restricting it to the maximal compact subgroups we get a morphism of symmetric pairs

$$
a:(\operatorname{Spin}(2 n), \operatorname{Spin}(2 n-1)) \longrightarrow\left(U\left(2^{n-1}\right) \times U\left(2^{n-1}\right), U\left(2^{n-1}\right)_{\text {diag }}\right)
$$

where $U\left(2^{n-1}\right)_{\text {diag }}$ is the diagonal subgroup. The last map is $a: u \longmapsto(u, \sigma(u))$ where $\sigma$ is the involution whose involutive subgroup is $\operatorname{Spin}(2 n-1)$.

The projections of $a(\operatorname{Spin}(2 n))$ to the first and second factors are half-spinor and anti-half-spinor representations. Indeed, the involution $\sigma$ of the Lie algebra $o(2 n, \mathbb{C})$ whose involutive subalgebra is $o(2 n-1, \mathbb{C})$ interchanges the half-spinor representations (Lemma 2.16).

So we get a commutative diagram:

$$
\begin{array}{ccc}
\operatorname{Spin}(2 n) & \stackrel{a}{\longrightarrow} & U(N) \times U(N) \\
\downarrow p_{1} & & \downarrow p_{2} \\
\frac{\operatorname{Spin}(2 n)}{\operatorname{Spin}(2 n-1)} & \stackrel{a^{\prime}}{\longrightarrow} & \frac{U(N) \times U(N)}{U(N)_{\text {diag }}}
\end{array}
$$

Under the map $p_{2}:\left(u_{1}, u_{2}\right) \rightarrow u_{1} u_{2}^{-1}$, the primitive generator $B_{2 n-1} \in$ $H_{\text {top }}^{2 n-1}(U(N))$ goes to $p_{2}^{*} B_{2 n-1}=B_{2 n-1} \otimes 1-1 \otimes B_{2 n-1}$.

The key topological statement is that $a^{\prime}\left(B_{2 n-1}\right) \neq 0$. It is a corollary of Proposition 2.15. To see this recall that there is the Euler class

$$
E_{2 n-1}:=\pi^{*}\left(\left[S^{2 n-1}\right]\right) \in H_{\text {top }}^{*}(S O(2 n))
$$

where $\pi: S O(2 n) \rightarrow S^{2 n-1}$ is the natural projection. There are the corresponding classes

$$
e_{2 n-1} \in H^{*}(o(2 n, \mathbb{C})) \quad \text { and } \quad \bar{e}_{2 n} \in H_{\text {top }}^{*}(B S O(2 n)) .
$$

One can identify $H_{\text {top }}^{*}(B S O(2 n))$ with the ring of invariant polynomials on the Lie algebra $o(2 n)$. Then the Euler class corresponds to (a nonzero multiple of) the Pfaffian. The constant $c_{n}$ can be calculated as follows. Set $E_{2 n-1}=\alpha_{n}[P f], B_{2 n-1}=$ $\beta_{n}\left[\operatorname{Tr} A^{n}\right]$ where $[P f]$ and $\left[\operatorname{Tr} A^{n}\right]$ are the topological cohomology classes of $S^{2 n-1}$ and $U(N)$ corresponding to the invariant symmetric polynomials given by the Pfaffian and $\operatorname{Tr} A^{n}$. Then $c_{n}=(-1)^{n(n-1) / 2} n ! \cdot \beta_{n} / \alpha_{n}$. Theorem 2.17 is proved.

Theorem 1.1 follows immediately from Theorems 2.17, 2.1 (in the compact case), 2.5 (in the noncompact case) and 2.12 and Lemma 2.11. First of all the image of the element $c\left(M^{2 n-1}\right) \in H_{2 n-1}\left(G L_{N}(\overline{\mathbb{Q}}) ; \mathbb{Q}\right)$ in $K_{2 n-1}(\overline{\mathbb{Q}})$ gives the invariant of $M^{2 n-1}$ we are looking for. It is well defined because $H_{2 n-1}(P(\overline{\mathbb{Q}}) ; \mathbb{Q})=\overline{\mathbb{Q}}^{*}$ projects to zero in $K_{2 n-1}(\overline{\mathbb{Q}})$ (notice that $2 n-1>1$ ). Further, we have an embedding

$$
\varphi:\left(S O(2 n-1,1)(\overline{\mathbb{Q}}), T_{n}(v)(\overline{\mathbb{Q}})\right) \hookrightarrow\left(G L_{N}(\overline{\mathbb{Q}}), P(\overline{\mathbb{Q}})\right) .
$$

The restriction of the cohomology class $b_{2 n-1}$ to $P(\overline{\mathbb{Q}})$ is zero, so it provides a relative class $\tilde{b}_{2 n-1}$. Similarly one has the relative Lobachevsky class $\tilde{v}_{2 n-1}$. It follows from Theorem 2.17 that $\varphi^{*}\left(\tilde{b}_{2 n-1}\right)=\tilde{v}_{2 n-1}$. According to Theorems 2.1, 2.17 and 2.12

$$
\begin{gathered}
\operatorname{vol}\left(M^{2 n-1}\right)=\left\langle\tilde{v}_{2 n-1}, \tilde{c}\left(M^{2 n-1}\right)\right\rangle=\left\langle\tilde{b}_{2 n-1}, \varphi_{*} \tilde{c}\left(M^{2 n-1}\right)\right\rangle \\
=\left\langle b_{2 n-1}, \varphi_{*} c\left(M^{2 n-1}\right)\right\rangle .
\end{gathered}
$$


Theorem 1.3 now follows from Theorem 1.1 and the main results of [G1] or [G2] where the Borel regulator on $K_{5}(F)$, where $F$ is a number field, was computed via the trilogarithm. See s. 3 and 5 in [G2]. Theorem 1.2 follows from Theorem 1.1 and results of s.2 in [G1].

Remark. The half-spinor representations seems to be the only ones among the fundamental representations of $S O(2 n-1,1)$ such that the restriction of the Borel class to $S O(2 n-1,1)$ is not zero. This means that only the half-spinor representations lead to a nontrivial invariant $c\left(M^{2 n-1}\right) \in H_{2 n-1}\left(G L_{N}(\overline{\mathbb{Q}}) ; \mathbb{Q}\right)$.

\section{Noneuclidean POLYhedrons AND MiXed Hodge STRUCtures}

1. The scissors congruence class of a hyperbolic manifold. We will use the following version of the definition of the scissors congruence groups.

Any $n+1$ points $x_{0}, \ldots, x_{n}$ in the space $V^{n}$ define a geodesic simplex $I\left(x_{0}, \ldots, x_{n}\right)$ with vertices in these points. $\mathcal{P}\left(V^{n}\right)$ is the abelian group generated by symbols $\left\{I\left(x_{0}, \ldots, x_{n}\right), \alpha\right\}$ where $\alpha$ is an orientation of $V^{n}$, subject to the following relations:

a) $\left\{I\left(x_{0}, \ldots, x_{n}\right), \alpha\right\}=0$ if $x_{0}, \ldots, x_{n}$ lie in a geodesic hyperplane.

b) $\left\{I\left(x_{0}, \ldots, x_{n}\right), \alpha\right\}=\left\{I\left(g x_{0}, \ldots, g x_{n}\right), g \alpha\right\}$ for any element $g$ form the group of automorphisms of the corresponding geometry.

c) $\left\{I\left(x_{\sigma(0)}, \ldots, x_{\sigma(n)}\right), \beta\right\}=-(-1)^{|\sigma|}\left\{I\left(x_{0}, \ldots, x_{n}\right), \alpha\right\}$ where $\sigma$ is a permutation and $\beta$ is another orientation of $V^{n}$.

d) $\sum_{i=0}^{n+1}(-1)^{i}\left\{I\left(x_{0}, \ldots, \hat{x}_{i}, \ldots, x_{n+1}\right), \alpha\right\}=0$ for any $n+2$ points $x_{i}$ in $V^{n}$.

The automorphism groups are: the group $O(n, 1)$ for the hyperbolic case, $O(n+1)$ for the spherical geometry and the semidirect product of $O(n)$ and translation group $E^{n}$ for the Euclidean geometry.

The Dehn invariant is defined as follows. Let $(I, \alpha)$ be a generator of $\mathcal{P}\left(V^{n}\right)$. For each $i$-dimensional edge $A$ of the geodesic simplex $I$ consider the corresponding geodesic $i$-plane. It inherits the same type of geometry as $V^{n}$ (i.e. it is a hyperbolic plane in hyperbolic geometry, etc.) and vertices of the edge $A$ define a geodesic simplex in it. The numeration of its vertices is induced by the one of $I$. Choose an orientation $\alpha_{A}$ of this $i$-plane. We get an element of $\mathcal{P}\left(V^{i}\right)$ that will be denoted as $I_{A}$.

Now look at an $(n-i)$-plane $A^{\prime}$ orthogonal to $A$ and intersecting it at a certain point $e$. This is a euclidean plane in all geometries. The intersection of the sphere in $A^{\prime}$ centered in $e$ with $i+1$-dimensional edges of $I$ containing $A$ defines a spherical simplex. Its vertices have a natural numeration induced by the numeration of the vertices of $I$. Finally, there is an orientation $\alpha_{A^{\prime}}$ of $A^{\prime}$ such that the orientation of $V^{n}$ defined by $\alpha_{A}$ and $\alpha_{A^{\prime}}$ coincides with the orientation $\alpha$. So we get an element $I_{A^{\prime}} \in \mathcal{P}\left(S^{n-i}\right)$. By definition

$$
D_{n}^{V}(I, \alpha):=\sum_{A} I_{A} \otimes I_{A^{\prime}}
$$

where the sum is over all edges of the simplex $I$ of dimension $i, 0<i<n$.

Notice that our groups $\mathcal{P}\left(S^{n}\right)$ are smaller than the ones defined in [Sah1]. For those groups one has $\mathcal{P}\left(S^{2 n}\right)=\mathcal{P}\left(S^{2 n-1}\right)$.

Lemma 3.1. $\mathcal{P}\left(S^{2 n}\right)=0$.

Proof. Points $-x_{0}, x_{0}, x_{1}, \ldots, x_{2 n-1}$ belong to a geodesic hyperplane in $S^{2 n}$. Therefore $\left\{I\left(-x_{0}, x_{0}, x_{1}, \ldots, x_{2 n-1}\right), \alpha\right\}=0$. 
Using this fact we see that the additivity axiom $\mathrm{d})$ for $\left(-x_{0}, x_{0},, x_{1}, \ldots, x_{2 n}\right)$ together with a) implies that $\left(I\left(x_{0}, x_{1}, \ldots, x_{2 n}\right), \alpha\right)=\left(I\left(-x_{0}, x_{1}, \ldots, x_{2 n}\right), \alpha\right)$ and hence $\left(I\left(x_{0}, x_{1}, \ldots, x_{2 n}\right), \alpha\right)=\left(I\left(-x_{0},-x_{1}, \ldots,-x_{2 n}\right), \alpha\right)$. The relation $\left.\mathrm{b}\right)$ for the antipodal involution is $\left(I\left(x_{0}, x_{1}, \ldots, x_{2 n}\right), \alpha\right)=\left(I\left(-x_{0},-x_{1}, \ldots,-x_{2 n}\right), \beta\right)$. Applying c) we get 3.1 .

Any hyperbolic manifold of finite volume can be cut into a finite number of geodesic simplices (see Section 2). If it is compact, these simplices have vertices in $\mathcal{H}^{n}$, and we take the sum of the corresponding elements in $\mathcal{P}\left(\mathcal{H}^{n}\right)$. If the manifold is noncompact, the vertices of these simplices might be on the absolute $\partial \mathcal{H}^{n}$. Consider the scissors congruence group $\mathcal{P}\left(\overline{\mathcal{H}}^{n}\right)$ generated by simplices with vertices at $\overline{\mathcal{H}}^{n}=$ $\mathcal{H}^{n} \cup \partial \mathcal{H}^{n}$. The natural inclusion

$$
\mathcal{P}\left(\mathcal{H}^{n}\right) \hookrightarrow \mathcal{P}\left(\overline{\mathcal{H}}^{n}\right)
$$

is an isomorphism ([Sah2]). The sum of the corresponding elements of $\mathcal{P}\left(\mathcal{H}^{n}\right)$ does not depend on the cutting. So any hyperbolic manifold $M^{n}$ produces an element $s\left(M^{n}\right) \in \mathcal{P}\left(\mathcal{H}^{n}\right)$.

Proposition 3.2. The Dehn invariant of $s\left(M^{n}\right)$ is equal to zero.

Proof. This is easy for compact manifolds. Namely, consider a $k$-dimensional edge $A$ of the triangulation of $V^{n}$ on geodesic simplices. Let $\left\{I^{i}\right\}$ be the set of all simplices containing the edge $A$. Each simplex $I^{i}$ defines an element $I_{A^{\prime}}^{i} \in \mathcal{P}\left(S^{n-k}\right)$ : the "inner angle" at the edge $A$ (see above). $I_{A}$ appears in the formula for $D_{n}^{H}\left(s\left(M^{n}\right)\right)$ with factor $\sum_{i} I_{A^{\prime}}^{i}$. But $\sum_{i} I_{A^{\prime}}^{i}=\left[S^{n-k}\right]=0$ in $\mathcal{P}\left(S^{n-k}\right)$ because $M^{n}$ is a manifold without boundary.

For noncompact manifolds, we have to proceed as follows. We define the extended Dehn invariant

$$
D_{2 n-1}^{H}: \mathcal{P}\left(\overline{\mathcal{H}}^{2 n-1}\right) \longrightarrow \bigoplus_{i=1}^{n-1} \mathcal{P}\left(\overline{\mathcal{H}}^{2 i-1}\right) \otimes \mathcal{P}\left(S^{2(n-i)-1}\right)
$$

which commutes with (27). The definition of the components of (28) corresponding to edges of dimension bigger than 1 is verbatim the same. For 1-dimensional edges we use the following Thurston's regularization procedure. Let $I\left(x_{1}, \ldots, x_{2 n}\right)$ be a simplex in $\mathcal{P}\left(\overline{\mathcal{H}}^{2 n-1}\right)$. For each infinite vertex $x_{i}$ delete a small horoball centered at $x_{i}$. Denote by $x_{i}(j)$ the intersection point of the edge $x_{i} x_{j}$ with the horosphere. If $x_{k}$ is a point inside hyperbolic space, set $x_{k}(j)=x_{k}$. Then the component of (28) corresponding to the edge $x_{i} x_{j}$ is the tensor product of the 1-dimensional hyperbolic simplex $x_{i}(j) x_{j}(i)$ and the spherical $2 n-1$ simplex that appears in the usual definition of Dehn invariant ("inner angle" at the edge $x_{i} x_{j}$, see above). The expression we get does not depend on the choice of horoballs because of the following reason. Consider a simplex in euclidean space $\mathbb{R}^{2 n-2}$. Each vertex $v$ defines a spherical simplex and hence an element of $\mathcal{P}\left(S^{2 n-2}\right)$ ("inner angle" at $v)$. Then the sum of the elements corresponding to all vertices of the simplex is zero (this generalises the fact that the sum of the angles of a euclidean triangle is $\pi$ and true only for simplices in even-dimensional spaces). After this the proof for noncompact hyperbolic manifolds is the same as for compact ones.

Say that a geodesic simplex is defined over $\overline{\mathbb{Q}}$ if it is equivalent under the motion group to a geodesic simplex which has vertices with coordinates in $\overline{\mathbb{Q}}$. 
Proposition 3.3. Any hyperbolic manifold can be decomposed on geodesic simplices defined over $\overline{\mathbb{Q}}$.

Corollary 3.4. The scissors congruence class of a hyperbolic manifold belongs to $\mathcal{P}(\mathcal{H}, \overline{\mathbb{Q}})$.

Proof. By Weil's rigidity theorem ([Ra] $) \pi_{1}(M)$ is conjugated to a subgroup whose entries are algebraic numbers. If $M$ is compact, let us take sufficiently many points whose coordinates are algebraic numbers inside of the fundamental domain. Consider the Dirichlet decomposition corresponding to these points and their orbits under the action of the group $\pi_{1}(M)$. The intersection of $n+1$ geodesic hyperplanes defined over $\overline{\mathbb{Q}}$ is a point defined over $\overline{\mathbb{Q}}$. So the vertices of this decomposition are defined over $\overline{\mathbb{Q}}$.

If $M$ has cusps, then their coordinates are also defined over $\overline{\mathbb{Q}}$, and the Dirichlet decomposition with respect to chosen points and the cusps gives the desired decomposition of $M$ on geodesic simplices defined over $\overline{\mathbb{Q}}$ (see also [EP]).

2. Hodge-Tate structures and the Hopf algebra $\mathcal{H}_{\bullet}$. For the convenience of the reader I recall some definitions from [BGSV]. For details and proofs see [G4], Section 4. Let us call a Hodge-Tate structure a mixed $\mathbb{Q}$ Hodge structure with weight factors isomorphic to $\mathbb{Q}(k)$. Say that $H$ is an $n$-framed Hodge-Tate structure if it is supplied with a nonzero vector in $g r_{2 n}^{W} H$ and a nonzero functional in $g r_{0}^{W} H$. Consider the finest equivalence relation on the set of all $n$-framed HodgeTate structures for which $H_{1} \sim H_{2}$ if there is a map $H_{1} \rightarrow H_{2}$ compatible with frames. For example any $n$-framed Hodge-Tate structure is equivalent to an $H$ with $W_{-2} H=0, W_{2 n} H=H$. Let $\mathcal{H}_{n}$ be the set of equivalence classes.

One may introduce on $\mathcal{H}_{n}$ the structure of an abelian group as follows. For $\left[H_{1}\right],\left[H_{2}\right] \in \mathcal{H}_{n}$ a vector in $g r_{2 n}^{W}\left(H_{1} \oplus H_{2}\right)$ will be the one whose components are distinguished vectors in $g r_{2 n}^{W} H_{1}$ and $g r_{2 n}^{W} H_{2}$. The frames in $g r_{0}^{W} H_{i}$ define the maps $g r_{0}^{W}\left(H_{1} \oplus H_{2}\right) \rightarrow \mathbb{Q}(0) \oplus \mathbb{Q}(0)$. Its composition with the sum map $\mathbb{Q}(0) \oplus \mathbb{Q}(0) \rightarrow \mathbb{Q}(0)$ will be a distinguished functional in $g r_{0}^{W} H$.

Let $-[H]$ be the class of $H$ in which the frame in $g_{2 n}^{W} H$ is multiplied by -1 . (We will get the same class by multiplying by -1 the functional in $g r_{0}^{W} H$.)

The tensor product of mixed Hodge structures induces the commutative multiplication

$$
\mu: \mathcal{H}_{k} \otimes \mathcal{H}_{\ell} \rightarrow \mathcal{H}_{k+\ell}
$$

Let us define the comultiplication

$$
\nu=\bigoplus_{k+\ell=n} \nu_{k \ell}: \mathcal{H}_{n} \rightarrow \bigoplus_{k+\ell=n} \mathcal{H}_{k} \otimes \mathcal{H}_{\ell} .
$$

Let $[H] \subset \mathcal{H}_{n}$, and let $\left(v_{n}, f_{0}\right)$ be a framing for $H: v_{n} \in g r_{2 n}^{W} H, f_{0} \in\left(g r_{0}^{W} H\right)^{*}$. Let $R \subset g r_{2 k}^{W} H$ be the lattice and $R^{*} \subset\left(g r_{2 k}^{W} H\right)^{*}$ the dual one. Define homomorphisms

$$
\varphi: R \rightarrow \mathcal{H}_{k}, \quad \psi: R^{*} \rightarrow \mathcal{H}_{n-k} .
$$

Namely, for $x \in R$ (resp. $\left.y \in R^{*}\right), \varphi(x)$ (resp. $\psi(y)$ ) is the class of the mixed Hodge structure $H$ with framing $\left(x, f_{0}\right)$ (resp. $H \otimes \mathbb{Q}(k)$ with the frames $\left.\left(v_{n}, y\right)\right)$.

Let $\left\{e_{j}\right\},\left\{e^{j}\right\}$ be dual bases in $R, R^{*}$. Then

$$
\nu_{k, n-k}([H]):=\sum_{j} \varphi\left(e_{j}\right) \otimes \psi\left(e^{j}\right)
$$


It is easy to see that $\nu(a \cdot b)=\nu(a) \cdot \nu(b)$. Here $a \cdot b=\mu(a \otimes b)$.

Lemma 3.5. The coproduct depends only on the equivalence class of framed HodgeTate structures. Therefore it provides a well-defined group homomorphism (29).

Proof. Suppose $H \rightarrow H^{\prime}$ is an injective morphism of Hodge-Tate structures respecting the frames. Let us show that $\nu_{k, n-k}\left(\left(H ; v_{0}, f_{n}\right)\right)=\nu_{k, n-k}\left(\left(H^{\prime} ; v_{0}^{\prime}, f_{n}^{\prime}\right)\right)$. We may suppose that $g r_{2 k}^{W} H=0$ if $k<0$ and $k>n$. If $g r_{2 k}^{W} H=g r_{2 k}^{W} H^{\prime}$, we have nothing to check. Choose the biggest $k$ such that $g r_{2 k}^{W} H \neq g r_{2 k}^{W} H^{\prime}$. Then there is an exact sequence of mixed Hodge structures

$$
0 \longrightarrow H / W_{2 k-2} H \longrightarrow H^{\prime} / W_{2 k-2} H^{\prime} \longrightarrow \bigoplus \mathbb{Q}(-k) \longrightarrow 0 .
$$

It splits since the weights of $H / W_{2 k-2} H$ are not less than $2 k$ and

$$
\operatorname{Ext}_{M H S}^{1}\left(\mathbb{Q}\left(-k^{\prime}\right), \mathbb{Q}(-k)\right)=0
$$

if $k^{\prime} \geq k$. So we proved the statement.

A similar argument (or dualization) is used when $H \rightarrow H^{\prime}$ is surjective. The lemma is proved.

So the abelian group $\mathcal{H}_{\bullet}:=\bigoplus \mathcal{H}_{n}$, where $\mathcal{H}_{0}:=\mathbb{Z}$, has a structure of graded Hopf algebra with the commutative multiplication $\mu$ and the comultiplication $\nu$.

The following result is the main reason to consider the Hopf algebra $\mathcal{H}_{\bullet}$. Proofs can be found in Section 4 of [G4].

Theorem 3.6. The category of mixed $\mathbb{Q}$-Hodge-Tate structures is canonically equivalent to the category of finite-dimensional graded $\mathcal{H}_{\bullet}$-comodules.

Namely, the equivalence assigns to a Hodge structure $H$ the graded comodule $M(H), M(H)_{n}=g r_{2 n}^{W}(H)$ with $\mathcal{H}_{\bullet}$-action $M(H) \otimes M(H)^{*} \longrightarrow \mathcal{H}_{\bullet}$ given by the formula $x_{m} \otimes y_{n} \longrightarrow$ class of mixed Hodge structure $H$ framed by $x_{m}, y_{n}$.

Let $\tilde{\nu}:=\nu-1 \otimes \mathrm{id}-\mathrm{id} \otimes 1$ be the reduced comultiplication. The cohomology of the Hopf algebra $\mathcal{H}_{\bullet}$ is computed as the cohomology of the cobar complex

$$
\mathcal{H}_{>0} \stackrel{\tilde{\nu}}{\longrightarrow} \mathcal{H}_{>0}^{\otimes 2} \longrightarrow \mathcal{H}_{>0}^{\otimes 3} \longrightarrow \ldots
$$

In particular we get formula (6).

3. The Hodge-Tate structure corresponding to a noneuclidean geodesic simplex. Let $Q \subset \mathbb{C P}^{2 n-1}$ be a nondegenerate quadric and $M=\left(M_{1}, \ldots, M_{2 n}\right)$ a simplex in generic position with respect to $Q$. In particular a geodesic geodesic simplex in $\mathcal{H}^{2 n-1}$ or $S^{2 n-1}$ provides such data; see s. 1.5. Recall that $h(Q, M)$ is the mixed Hodge-Tate structure on $H^{2 n-1}\left(\mathbb{C P}^{2 n-1} \backslash Q, M ; \mathbb{Q}\right)$. The weights are from 0 to $2 n$. There are canonical isomorphisms

$$
\begin{gathered}
\mathbb{Q}(0)=H^{2 n-1}\left(\mathbb{C P}^{2 n-1}, M ; \mathbb{Q}\right) \longrightarrow W_{0} H^{2 n-1}\left(\mathbb{C P}^{2 n-1} \backslash Q, M ; \mathbb{Q}\right), \\
g r_{2 n}^{W} H^{2 n-1}\left(\mathbb{C P}^{2 n-1} \backslash Q, M ; \mathbb{Q}\right) \longrightarrow H^{2 n-1}\left(\mathbb{C P}^{2 n-1} \backslash Q ; \mathbb{Q}\right)=\mathbb{Q}(-n) .
\end{gathered}
$$

The cohomology class $\left[w_{Q}\right]$ of the meromorphic $(2 n-1)$-form

$$
w_{Q}:= \pm i^{n} \cdot \sqrt{\operatorname{det} \tilde{Q}} \cdot \frac{\sum_{i=1}^{2 n}(-1)^{i} x_{i} d x_{i} \wedge \ldots \wedge \widehat{d x}_{i} \wedge \ldots \wedge d x_{2 n}}{\tilde{Q}^{n}}
$$


has the following geometrical interpretation. The quadric $Q$ has 2 families of maximal isotopic subspaces. Let us denote by $\alpha_{Q}$ and $\beta_{Q}$ the corresponding cohomology classes in $H^{2 n-2}(Q)$. The exact sequence

$$
\ldots \rightarrow H^{2 n-1}\left(\mathbb{C P}^{2 n-1} \backslash Q\right) \stackrel{\delta}{\longrightarrow} H_{Q}^{2 n}\left(\mathbb{C P}^{n}\right)=H^{2 n-2}(Q)(-1) \longrightarrow H^{2 n}\left(\mathbb{C P}^{n}\right) \rightarrow \ldots
$$

shows that $\alpha_{Q}-\beta_{Q} \in \operatorname{Im} \delta$. In fact $\alpha_{Q}-\beta_{Q}$ is proportional to $\delta\left(\left[w_{Q}\right]\right)$. So to choose a sign in (32) one has to choose one of the families of maximal isotropic subspaces in $Q$.

Further, an orientation of the simplex $M$ (provided by the numeration of hyperplanes $\left.M_{i}\right)$ corresponds to a generator of $H_{2 n-1}\left(\mathbb{C P}^{2 n-1}, M\right)$. Therefore if we choose a sign of $w_{Q}$ and a simplex $M$, then the mixed Hodge structure $h(Q, M)$ is $n$-framed by $\left[w_{Q}\right] \in g r_{2 n}^{W} h(Q, M)$ and $\Delta_{M} \in g r_{0}^{W} h(Q, M)$.

For any subset $I=\left\{i_{1}, \ldots, i_{k}\right\} \subset\{0,1, \ldots, 2 n\}$ let $M_{I}:=M_{i_{1}} \cap \ldots \cap M_{i_{k}}$.

4. The Hopf algebra $S(\mathbb{C})$.. Let $(Q, \alpha)$ be a nondegenerate quadric $Q \in \mathbb{C P}^{2 n-1}$ together with a choice of one of the families of maximal isotropic subspaces on $Q$ denoted by $\alpha$.

Let us denote by $S_{n}(\mathbb{C})$ the abelian group generated by generic pairs $[(Q, \alpha) ; M]$ subject to the following relations:

R0) If $M_{0} \cap \ldots \cap M_{2 n-1} \neq \emptyset$, then $[(Q, \alpha) ; M]=0$.

R1) (Projective invariance) For any projective transformation $g \in P G L_{2 n}(\mathbb{C})$

$$
[(Q, \alpha) ; M]=[(g Q, g \alpha) ; g M] .
$$

R2) (Skew symmetry) a) For any permutation $\sigma$ of the set $\{0, \ldots, 2 n-1\}$ one has

$$
[(Q, \alpha) ; M]=(-1)^{|\sigma|}\left[(Q, \alpha) ; M_{\sigma}\right]
$$

where $M_{\sigma}=\left(M_{\sigma(0)}, \ldots, M_{\sigma(2 n-1)}\right)$.

b) Let $\beta$ be another family of maximal isotropic subspaces on the quadric $Q \in$ $\mathbb{C P}^{2 n-1}$. Then

$$
[(Q, \alpha) ; M]=-[(Q, \beta) ; M]
$$

R3) (Additivity) Let $M_{0}, \ldots, M_{2 n}$ be hyperplanes such that $Q \cap M_{I}$ is a nondegenerate quadric for any subset $I \in\{0, \ldots, 2 n\}$. Set $M^{(j)}:=\left(M_{0}, \ldots, \hat{M}_{j}, \ldots, M_{2 n}\right)$. Then

$$
\sum_{j=0}^{2 n+1}(-1)^{j}\left[(Q, \alpha) ; M^{(j)}\right]=0 .
$$

For example, $S_{1}(\mathbb{C})$ is generated by 4 -tuples of points $\left(q_{0}, q_{1}, m_{0}, m_{1}\right)$ on $\mathbb{C P}^{1}$. Here by definition $\alpha=q_{0}$. The cross-ratio defines an isomorphism $r: S_{1}(\mathbb{C}) \rightarrow \mathbb{C}^{*}$.

Remark. The ordering of points and relation R2)a) is necessary to have this isomorphism. Moreover if we have it, the orientation of the quadric $Q$ together with relation R2)a) in all dimensions is needed in order to define comultiplication $\Delta$.

Recall that there is an $n$-framed Hodge-Tate structure $h[(Q, \alpha) ; M]$ related to $H^{2 n-1}\left(\mathbb{C P}^{2 n-1} \backslash Q, M ; \mathbb{Q}\right)$ and a choice of a family of maximal isotropic subspaces $\alpha$ on $Q$.

Proposition 3.7. There is a homomorphism of abelian groups $h: S_{n}(\mathbb{C}) \rightarrow \mathcal{H}_{n}$ defined on generators as $h:[(Q, \alpha) ; M] \longrightarrow h[(Q, \alpha) ; M]$. 
Proof. We have to show that $h$ maps relations to zero. For R1) and R2) this is clear from the definitions. If $\bigcap M_{i} \neq \emptyset$, then $\operatorname{gr}_{0}^{W} h[(Q, \alpha) ; M]=0$ and so this $n$-framed Hodge-Tate structure is equivalent to zero.

Let us prove that

$$
\sum_{j=0}^{2 n}(-1)^{j} h\left[(Q, \alpha), M^{(j)}\right]=0 .
$$

Set

$$
H^{\prime}:=H^{2 n-1}\left(\mathbb{C P}^{2 n-1} \backslash Q, \bigcup_{i=0}^{2 n} M_{i} ; \mathbb{Q}\right) .
$$

Then there are canonical morphisms of mixed Hodge structures

$$
\begin{aligned}
H^{\prime} \longrightarrow H_{-} & :=\bigoplus_{j: \text { odd }} H^{2 n-1}\left(\mathbb{C P}^{2 n-1} \backslash Q, M^{(j)} ; \mathbb{Q}\right), \\
H^{\prime} \longrightarrow H_{+} & :=\bigoplus_{j: \text { even }} H^{2 n-1}\left(\mathbb{C P}^{2 n-1} \backslash Q, M^{(j)} ; \mathbb{Q}\right) .
\end{aligned}
$$

There are natural frames in $H_{-}$and $H_{+}$, and, using the fact that

$$
\sum_{j: \text { odd }} \Delta_{M^{(j)}}=\sum_{j: \text { even }} \Delta_{M^{(j)}} \text { in } H_{2 n-1}\left(\mathbb{C P}^{2 n-1}, \bigcup_{i=0}^{2 n} M_{i}\right)
$$

we can introduce a frame in $H$ such that these morphisms will respect the frames, providing the equivalence $H_{-} \sim H_{+}$of the $n$-framed Hodge-Tate structures. Proposition 3.7 is proved.

Set $S_{0}(\mathbb{C})=\mathbb{Z}$ and $S(\mathbb{C})$ : := $\bigoplus_{n=0}^{\infty} S_{n}(\mathbb{C})$. Our next goal is to define on $S(\mathbb{C})$. a structure of graded commutative Hopf algebras.

The product of elements $[(Q, \alpha) ; M] \in S_{n}(\mathbb{C})$ and $\left[\left(Q^{\prime}, \alpha^{\prime}\right) ; M^{\prime}\right] \in S_{2 n^{\prime}}(\mathbb{C})$ is defined as follows. Let $V$ be a vector space of dimension $2 n$. Choose a quadratic equation $\tilde{Q}$ of the quadric $Q$ and a maximal isotropic subspace $\tilde{\alpha}$ from the family $\alpha$. Denote by $\tilde{M}_{i}$ the codimension 1 subspace projected to $M_{i}$. There is analogous data in a vector space $V^{\prime}$ related to $\left[\left(Q^{\prime}, \alpha^{\prime}\right) ; M^{\prime}\right]$. Then in $V \oplus V^{\prime}$ there are the quadratic form $\tilde{Q} \oplus \tilde{Q}^{\prime}$, the coordinate simplex consisting of subspaces $\tilde{M}_{i} \oplus V^{\prime}$ and $V \oplus \tilde{M}_{\bar{i}}^{\prime}$ and the maximal isotropic subspace $\tilde{\alpha} \oplus \tilde{\alpha}^{\prime}$. The projectivisation of this data defines a pair $\left[\left(Q * Q^{\prime} ; \alpha * \alpha^{\prime}\right), M * M^{\prime}\right]$. Its projective equivalence class does not depend on the choice of $\tilde{Q}, \tilde{Q}^{\prime}$ and $\tilde{\alpha}, \tilde{\alpha}^{\prime}$. By definition the product

$$
[(Q, \alpha) ; M] *\left[\left(Q^{\prime}, \alpha^{\prime}\right) ; M^{\prime}\right] \in S_{2\left(n+n^{\prime}\right)}(\mathbb{C})
$$

is the class of this pair.

Proposition 3.8. The product is well defined.

Proof. The proof is clear from the definitions. For example to check

$$
\sum_{j=0}^{2 n}(-1)^{j}\left[(Q, \alpha) ; M^{j}\right] *\left[\left(Q^{\prime}, \alpha^{\prime}\right) ; M^{\prime}\right]=0
$$

consider $2\left(n+n^{\prime}\right)+1$ hyperplanes in $P\left(V^{\prime}\right)$ corresponding to $\left\{M_{i} \oplus V^{\prime}\right\} \cup\left\{V \oplus M_{\bar{i}}^{\prime}\right\}$ where $0 \leq i \leq 2 n$ and $0 \leq i^{\prime} \leq 2 n-1$. Then on the level of generators the left-hand side is just the additivity relation written for $\left(Q * Q^{\prime} ; \alpha * \alpha^{\prime}\right)$ and these hyperplanes. 
Now let us define the comultiplication

$$
\Delta: S_{n}(\mathbb{C}) \longrightarrow \bigoplus_{k=0}^{n} S_{k}(\mathbb{C}) \otimes S_{(n-k)}(\mathbb{C})
$$

Let $[(Q, \alpha) ; M]$ be a generator of $S_{n}(\mathbb{C})$. If $\bigcap M_{i} \neq \emptyset$, then $\Delta[(Q, \alpha) ; M]=0$ by definition. Now let us suppose that $\bigcap M_{i}=\emptyset$. Choose a data $(\tilde{Q}, \tilde{\alpha}) ;\left\{\tilde{M}_{i}\right\}$ in a vector space $V$ corresponding to $[(Q, \alpha) ; M]$. Let $\tilde{Q}_{I}$ be the restriction of the quadratic form $\tilde{Q}$ to the subspace $\tilde{M}_{I}$. Set $\tilde{\alpha}_{I}:=\tilde{\alpha} \cap M_{I}$.

Let $\bar{I}:=\left\{\bar{i}_{1}<\ldots<\bar{i}_{2(n-k)}\right\}$ be the complement to $I$ in $\{0, \ldots, 2 n-1\}$. Then there is the family of hyperplanes $M_{\bar{i}_{1}} \cap M_{I}, \ldots, M_{\bar{i}_{2(n-k)}} \cap M_{I}$ in $M_{I}$ that will be reffered to as $M_{\{\bar{I}\}}$.

Further, let $\tilde{Q}^{I}$ (resp. $\tilde{\alpha}^{I}$ ) be the quadratic form (resp. maximal isotropic subspace) induced in $V / M_{I}$ by $\tilde{Q}$ (resp. $\tilde{\alpha}$ ). The hyperplanes $M_{i}$, where $i \in I$, induce the family $M^{I}$ of hyperplanes in $P\left(V / \tilde{M}_{I}\right)$. Set $\Delta=\bigoplus_{k=0}^{n} \Delta_{n-k, k}$ and

$$
\begin{gathered}
\Delta_{n-k, k}([Q, \alpha] ; M):= \\
\sum_{|I|=2 k}\left(\left[\left(\tilde{Q}^{I}, \tilde{\alpha}^{I}\right) ; \tilde{M}^{I}\right]\right) \otimes\left[\left(\tilde{Q}_{I}, \tilde{\alpha}_{I}\right) ; \tilde{M}_{\bar{I}}\right] \in S_{k}(\mathbb{C}) \otimes S_{(n-k)}(\mathbb{C}) .
\end{gathered}
$$

There is also a duality $i: S_{n}(\mathbb{C}) \rightarrow S_{n}(\mathbb{C})$ defined as follows. A nondegenerate quadric $Q \in \mathbb{C P}^{m}$ provides an isomorphism $i_{Q}: \mathbb{C P}^{m} \rightarrow \hat{\mathbb{C}} \mathbb{P}^{m}$. Namely, let $x \in \mathbb{C P}^{m}$. Consider the set of lines through $x$ tangent to $Q$. The tangency locus is a section of $Q$ by a hyperplane $i_{Q}(x)$. Let $v_{j}=\bigcap_{i \neq j} M_{i}$ be the vertices of simplex $M$. Set $\hat{M}:=\left(i_{Q}\left(v_{0}\right), \ldots, i_{q}\left(v_{m}\right)\right)$. Then

$$
i:[(Q, \alpha) ; M] \longrightarrow[(Q, \beta) ; \hat{M}]
$$

Theorem 3.9. a) The comultiplication $\Delta$ is well defined.

b) The multiplication $*$ and the comultiplication $\Delta$ provide the structure of graded commutative Hopf algebras on $S(\mathbb{C})$.

c) The duality $i$ is an antipode, i.e. $i(a * b)=i(a) * i(b) ; \Delta(i(a))=t(i \otimes i) \Delta(a)$, where $t$ interchanges factors in the tensor product.

Proof. a) We have to check that $\Delta_{k, n-k}$ takes relations R0)-R3) to zero. This is obvious for R1)-R2) and true by definition for R0). It remains to check R3).

It is convenient to extend the definition of $\Delta$ to generators $[(Q, \alpha) ; M]$ with $\bigcap M_{i} \neq \emptyset$ using (33) where the summation is over all subsets $I$ such that $|I|=2 k$, $\operatorname{codim} M_{I}=2 k$ and $M_{I^{\prime}} \neq M_{I}$ for any $I^{\prime} \supset I$ with $I^{\prime} \neq I$. It is obvious that then $\Delta[(Q, \alpha) ; M]=0$ because the second factor in (33) will always be 0 thanks to $\mathrm{R} 0)$.

Now let $M_{0}, \ldots, M_{2 n}$ be hyperplanes in $\mathbb{C P}^{2 n-1}$ such that $Q \cap M_{J}$ is a nondegenerate quadric for any subset $J$.

We have to prove that

$$
\Delta_{k, n-k}\left(\sum_{j=0}^{2 n}(-1)^{j}\left[(Q, \alpha) ; M^{(j)}\right]\right)=0 .
$$

Compute the left side by using definition (33). The formula we get is a sum of contributions corresponding to codimension $2 k$ edges of the configuration $\left(M_{0}, \ldots, M_{2 n}\right)$. Consider one of the contributions related to a certain edge $E$. Let $I$ be the set of all indices $i$ such that $E \subset M_{i}$. If $|I| \geq 2 k+2$, then all simplices $M^{(j)}$ are degenerate 
and so R3) is provided by R0). Suppose that $I=2 k+1$. Then in the part of the formula under consideration the second factor in all summands is the same, and the sum of the first factors is 0 thanks to relation R3). Similary if $I=2 k$ the first factor is the same, and the sum of the second ones is 0 according to R3).

b) We have to prove that $\Delta(a * b)=\Delta(a) * \Delta(b)$. This is an immediate consequence of the definitions and the following

Lemma 3.10. Let $V=V_{1} \oplus V_{2}, \tilde{Q}=\tilde{Q}_{1} \oplus \tilde{Q}_{2}, \tilde{M}=\tilde{M}_{1} \oplus \tilde{M}_{2}$. Suppose that $\operatorname{dim} V_{i}$ is odd for $i=1,2$. Then the element of $S(C)$ • corresponding to $([Q, \alpha] ; M)$ is equal to zero modulo 2-torsion.

Proof. The transformation $g=\left(-\mathrm{id}_{V_{1}}, \mathrm{id}_{V_{2}}\right)$ has determinant -1 and so interchanges connected components of the manifold of maximal isotropic subspaces of the quadric $Q$ and the simplex $M$. Therefore

$$
([Q, \alpha] ; M)=([g Q, g \alpha] ; g M)=([Q, \beta] ; M)=-([Q, \alpha] ; M) .
$$

Lemma 3.10 is proved.

The coassociativity of $\Delta$ also follows immediately from the definitions.

c) Clear from the definitions.

Remark (compare with the remark in s. 3.4). If we omit from the definition of the group $S_{n}(\mathbb{C})$ orientation of the quadric $Q$ and hence relation R2)a) we would miss Lemma 3.10 and therefore would get $\Delta(a * b) \neq \Delta(a) * \Delta(b)$.

The comultiplication $\Delta$ can be defined in terms of projective geometry. Namely $Q_{I}$ and $M_{\{\bar{I}\}}$ are the quadric and the simplex in $M_{I}$. To define the second factor in (33) consider the projective space formed by hyperplanes containing $M_{I}$. The tangency condition with $Q$ defines a quadric in this projective space. Hyperplanes $M_{i}, i \in I$, can be considered as vertices of a simplex in it. Finally an orientation of the quadric $Q$ provides orientations of both quadrics obtained.

According to Proposition 3.7 we have the morphism of abelian groups

$$
h: S(\mathbb{C}) \bullet \rightarrow \mathcal{H}_{\bullet} .
$$

Theorem 3.11. $h$ is a homomorphism of Hopf algebras.

Proof. Let us prove that $h$ commutes with comultiplication. We need the following elementary facts about the cohomology of quadrics.

a) If $Q \in \mathbb{C P}^{m}$ is a nonsingular quadric, then $H^{i}\left(\mathbb{C P}^{m} \backslash Q ; \mathbb{Q}\right)=0$ if $m$ is even, $i>0$ or $m$ is odd, $i \neq 2 n-1$ and $H^{2 n-1}\left(\mathbb{C P}^{2 n-1} \backslash Q ; \mathbb{Q}\right)=\mathbb{Q}(-n)$.

b) If $Q_{0}$ is a singular quadric in $\mathbb{C}^{m}$, then $H^{i}\left(\mathbb{C}^{m}, Q_{0} ; \mathbb{Q}\right)=0$ for all $i$.

Let us compute

$$
H^{*}\left(\mathbb{C P}^{2 n-1} \backslash Q, M \backslash(M \cap Q) ; \mathbb{Q}\right) .
$$

Notice that $(Q, M)$ is a normal crossing divisor. Recall that for a subset $I \in$ $\{0, \ldots, 2 n-1\}$ we set $Q_{I}:=M_{I} \cap Q$. Consider the corresponding simplicial scheme

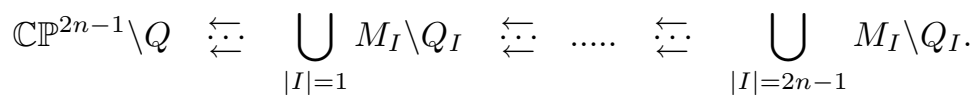

It produces the spectral sequence with

$$
E_{1}^{p, q}=H^{p}\left(M_{I} \backslash Q_{I} ; \mathbb{Q}\right) \quad \text { where }|I|=q .
$$


This spectral sequence degenerates at $E_{1}$ (because of the weight considerations). The filtration it induces on (34) coincides with the weight filtration. Therefore

$$
g r_{2(n-k)}^{W} H^{2 n-1}\left(\mathbb{C P}^{2 n-1} \backslash Q, M ; \mathbb{Q}\right)=\bigoplus_{|I|=2 k} H^{2(n-k)-1}\left(M_{I} \backslash Q_{I} ; \mathbb{Q}\right) .
$$

Moreover, for each subset $I$ with $|I|=2 k$ there is an obvious injective morphism of mixed Hodge structures

$$
i_{I}: h\left(Q_{I}, M_{I}\right) \rightarrow h(Q, M) .
$$

Recall that there are canonical up to a sign vectors $\left[\omega_{Q_{I}}\right] \in g r_{2(n-k)}^{W} h\left(Q_{I}, M_{I}\right)$. If we choose signs, their images form a basis in $\operatorname{gr}_{2(n-k)}^{W} h(Q, M)$. Further, there are morphisms of mixed Hodge structures

$$
H^{2 n-1}\left(\mathbb{C P}^{2 n-1} \backslash Q, M ; \mathbb{Q}\right) \longrightarrow H^{2 n-1}\left(\mathbb{C P}^{2 n-1} \backslash Q, \bigcup_{i \in I} M_{i} ; \mathbb{Q}\right) .
$$

Proposition 3.12. One has a canonical isomorphism of mixed Hodge structures

$$
H^{2 n-1}\left(\mathbb{C P}^{2 n-1} \backslash Q, \bigcup_{i \in I} M_{i} ; \mathbb{Q}\right)(n-k)=h\left(M^{I}, Q^{I}\right) .
$$

Proof. By Poincaré duality

$$
H^{2 n-1}\left(\mathbb{C P}^{2 n-1} \backslash Q, \bigcup_{i \in I} M_{i} ; \mathbb{Q}\right)=H^{2 n-1}\left(\mathbb{C P}^{2 n-1} \backslash \bigcup_{i \in I} M_{i}, Q ; \mathbb{Q}\right)^{*}(-(2 n-1)) .
$$

Notice that

$$
H^{2 n-1}\left(\mathbb{C P}^{2 n-1} \backslash Q, \bigcup_{i \in I} M_{i} ; \mathbb{Q}\right)=R^{2 n-1} f_{*}\left(j_{M *} j_{Q} ! \mathbb{Q}\right)
$$

where

$$
\mathbb{C P}^{2 n-1} \backslash\left(\bigcup_{i \in I} M_{i} \cup Q\right) \stackrel{j_{Q}}{\hookrightarrow} \mathbb{C P}^{2 n-1} \backslash\left(\bigcup_{i \in I} M_{i}\right) \stackrel{j_{M}}{\hookrightarrow} \mathbb{C P}^{2 n-1} \backslash M_{I}
$$

and $f$ is a composition

$$
\mathbb{C P}^{2 n-1} \backslash M_{I} \stackrel{f_{1}}{\longrightarrow} P\left(V / \tilde{M}_{I}\right) \backslash M^{I} \stackrel{f_{2}}{\longrightarrow} * .
$$

Let us compute $R^{2 n-1} f_{1 *}\left(j_{M *} j_{Q !} \mathbb{Q}\right)$. A point $x$ of the plane $P\left(V / \tilde{M}_{I}\right)$ corresponds to a plane $H_{x}$ of dimension $\operatorname{dim} M_{I}+1$ containing $M_{I}$. Set $h_{x}:=H_{x} \backslash M_{I}$. The fiber

$$
i_{x}^{*} R^{i} f_{1 *}\left(j_{M *} j_{Q !} \mathbb{Q}\right)
$$

is isomorphic to $H^{i}\left(h_{x}, h_{x} \cap Q\right)$. Recall that $x$ belongs to the quadric if and only if $h_{x} \cap Q$ is singular. So the fiber (36) at such points is zero according to b). The fiber (36) at points $x \in P\left(V / \tilde{M}_{I}\right) \backslash \mathbb{Q}^{I} \cap M^{\{I\}}$ is $Q(-k)$ for $i=2 k$ and zero for other $i$. Therefore

$$
R f_{1 *}\left(j_{M *} j_{Q !} \mathbb{Q}\right)=j_{M^{I} *} j_{Q^{I} !} \mathbb{Q}(-k)[-2 k]
$$

and applying again Poincaré duality we get Proposition 3.12. 
5. A remark on a symplectic approach to the Hopf algebra $S(\mathbb{C})$.. Let $W_{2 n}$ be a symplectic vector space decomposed to a direct sum of Lagrangian subspaces $W_{2 n}=E \oplus F$. Suppose also that coordinate hyperplanes $E_{1}, \ldots, E_{n}$ in $E$ are given. The symplectic structure provides an isomorphism $F=E^{*}$ and therefore there are dual hyperplanes $F_{1}, \ldots, F_{n}$ in $F$.

The subgroup of $S p\left(W_{2 n}\right)$ preserving this data is an $n$-dimensional torus $T_{n}$ (a maximal Cartan subgroup).

Let $L_{n}^{0}$ be the manifold of all Lagrangian subspaces $H \in W_{2 n}$ in generic position with respect to coordinate hyperplanes $E_{i} \oplus F, E \oplus F_{j}$. Each of them can be considered as a graph of a map $h: E \rightarrow F$ and hence defines a bilinear form $h \in E^{*} \otimes F^{*}$. The condition that $H$ is isotropic just means that $h$ is a symmetric bilinear form.

Lemma 3.13. Points of $L_{n}^{0} / T_{n}$ are in 1-1 correspondence with configurations (i.e. projective equivalence classes) of pairs $(Q ; M)$ where $Q$ is a nondegenerate quadric and $M$ is a simplex in generic position with respect to $Q$ in $P(E)$.

Proof. The coordinate hyperplanes $E_{1}, \ldots, E_{n}$ define a simplex in $P(E)$. The torus $T_{n}$ is the subgroup of all transformations in $G L(E)$ preserving the simplex. The lemma follows from these remarks.

\section{PERIODS}

Recall that $\mathcal{H}_{n}$ is the $\mathbb{Q}$-space of an $n$-framed Hodge-Tate structure over $\mathbb{Q}$. We will construct a canonical homomorphism, the big period map

$$
P_{n}: \mathcal{H}_{n} \quad \longrightarrow \mathbb{C} \otimes \mathbb{Q} \mathbb{C}_{\mathbb{Q}}^{*}(n-2) .
$$

Let $\tilde{\nu}_{n}$ be the restriction of the (restricted) coproduct $\tilde{\nu}$ to $\mathcal{H}_{n}$. There are canonical identifications

$$
\operatorname{Ker}\left(\tilde{\nu}_{n}\right)=\frac{\mathbb{C}}{(2 \pi i)^{n} \mathbb{Q}}=\operatorname{Ext}_{M H S / \mathbb{Q}}^{1}(\mathbb{Q}(0), \mathbb{Q}(n)) .
$$

The restriction of $P_{n}$ to the subgroup $\operatorname{Ker}\left(\tilde{\nu}_{n}\right)$ lands in $2 \pi i \otimes \mathbb{C}^{*}(n-2)$, and the map

$$
\frac{\mathbb{C}}{(2 \pi i)^{n} \mathbb{Q}}=\operatorname{Ker}\left(\tilde{\nu}_{n}\right) \quad \longrightarrow 2 \pi i \otimes \mathbb{C}^{*}(n-2)=\frac{\mathbb{C}}{(2 \pi i)^{n} \mathbb{Q}}
$$

is an isomorphism. Let $\operatorname{Im}(a+i b):=i b$. There is a canonical map

$$
\mathbb{C} \otimes \mathbb{C}^{*}(n-2) \longrightarrow \mathbb{R}(n-1), \quad a \otimes b \otimes(2 \pi i)^{n-2} \longrightarrow(2 \pi i)^{n-2} \operatorname{Im}(a) \cdot \log |b| .
$$

Combining $P_{n}$ with this map we get the homomorphism

$$
p_{n}: \mathcal{H}_{n} \quad \longrightarrow \mathbb{R}(n-1),
$$

called the real period map.

Remark. There exists another canonical real period map $q_{n}: \mathcal{H}_{n} \longrightarrow \mathbb{R}$ which kills the products: $q_{n+m}\left(H_{n} \cdot H_{m}\right)=0$ ([BD1]). It can also be recovered from the big period map; see [G5]. 
1. The maximal period. Let $\left(H ; v_{0}, f_{n}\right)$ be a Hodge-Tate structure over $\mathbb{Q}$, framed by $\mathbb{Q}(0)$ and $\mathbb{Q}(n)$, so $H(-n)$ is $n$-framed in the terminology of s. 3.2 . Choose a splitting over $\mathbb{Q}$ of the weight filtration on $H_{\mathbb{Q}}$. Then one can define the (maximal) period $p(H) \in \mathbb{C}$ as follows. Using the splitting we lift the frame vector $v_{0} \in g r_{0}^{W} H_{\mathbb{Q}}$ to a vector $v_{0}^{\prime} \in W_{0} H_{\mathbb{Q}}$ which maps to $v_{0}$ under the projection $W_{0} H_{\mathbb{Q}} \longrightarrow g r_{0}^{W} H_{\mathbb{Q}}$. Projecting

$$
W_{0} H_{\mathbb{C}} \longrightarrow W_{0} H_{\mathbb{C}} / W_{-2 n-1} H_{\mathbb{C}} \longrightarrow g r_{F}^{-n} H_{\mathbb{C}},
$$

we get a vector $v_{0}^{\prime \prime} \in g r_{F}^{-n} H_{\mathbb{C}}$ out of $v_{0}^{\prime}$. Applying the frame functional $f_{-n}$ : $g r_{F}^{-n} H_{\mathbb{C}}=g r_{-2 n}^{W} H_{\mathbb{C}} \longrightarrow \mathbb{C}$ to $v_{0}^{\prime \prime}$ we obtain a number $p\left(H ; v_{0}, f_{-n}\right) \in \mathbb{C}$, called the period of the splitted framed Hodge-Tate structure $\left(H ; v_{0}, f_{n}\right)$.

We define an equivalence relation on the set of all splitted framed Hodge-Tate structures as the finest equivalence relation for which any morphism of mixed Hodge structure $H \rightarrow H^{\prime}$ respecting the splittings and the frames is an equivalence.

Let $\tilde{\mathcal{H}}_{n}$ be the set of equivalence clases of splitted $n$-framed Hodge-Tate structures. Then $\tilde{\mathcal{H}}_{\bullet}:=\bigoplus_{n} \tilde{\mathcal{H}}_{n}$ is equipped in the usual way (see s. 3.2 ) with a structure of a graded Hopf algebra. For instance $\tilde{\mathcal{H}}_{1}=\mathbb{C} \otimes \mathbb{Q}$. In particular there is a coproduct map $\nu: \tilde{\mathcal{H}}_{\bullet} \rightarrow \tilde{\mathcal{H}}_{\bullet} \otimes \tilde{\mathcal{H}}_{\bullet}$.

Let $H \rightarrow H^{\prime}$ be a morphism of Hodge-Tate structures respecting the frames and splittings. Then the maximal periods of $H$ and $H^{\prime}$ are obviously the same, so we get the period homomorphism $\tilde{p}_{n}: \tilde{\mathcal{H}}_{n} \rightarrow \mathbb{C}$.

Lemma 4.1. The period map is multiplicative, i.e. it provides a homomorphism of algebras $\tilde{p}: \tilde{\mathcal{H}}_{\bullet} \rightarrow \mathbb{C}$.

Proof. Clear from the definitions.

2. The period operator and the period matrix. Let $H$ be a mixed HodgeTate structure over $\mathbb{Q}$. Then there is an isomorphism

$$
H_{\mathbb{C}}=\bigoplus_{p} F^{p} H_{\mathbb{C}} \cap W_{2 p} H_{\mathbb{C}}
$$

Notice that the canonical map

$$
F^{p} H_{\mathbb{C}} \cap W_{2 p} H_{\mathbb{C}} \longrightarrow g r_{2 p}^{W} H_{\mathbb{Q}} \otimes_{\mathbb{Q}} \mathbb{C}
$$

is an isomorphism. Using the isomorphisms (39) and (38) we produce a canonical morphism

$$
S_{H T}: \bigoplus_{p} g r_{2 p}^{W} H_{\mathbb{Q}} \longrightarrow H_{\mathbb{C}}
$$

On the other hand, a splitting of the weight filtration on $H_{\mathbb{Q}}$ also provides us with a morphism

$$
S_{W}: \bigoplus_{p} g r_{2 p}^{W} H_{\mathbb{Q}} \longrightarrow H_{\mathbb{C}} .
$$

Both maps become isomorphisms when extended to $\bigoplus_{p} g r_{2 p}^{W} H_{\mathbb{C}}$. Therefore a splitting of the weight filtration on $H_{\mathbb{Q}}$ provides a map

$$
S_{H T}^{-1} \circ S_{W}: \bigoplus_{p} g r_{2 p}^{W} H_{\mathbb{C}} \longrightarrow \bigoplus_{p} g r_{2 p}^{W} H_{\mathbb{C}}
$$

We call it the period operator.

Let us choose a basis in each $\mathbb{Q}$-vector space $g r_{2 p}^{W} H_{\mathbb{Q}}$, thus providing a basis in their direct sum. We define the period matrix as the matrix of the period operator 
written in such a basis. One can define a mixed Hodge-Tate structure by exhibiting its period matrix. See an example in s. 4.5.

3. The big period map. Let $V$ be a $\mathbb{Q}$-vector space. Denote by $\left\{v_{k}\right\}$ a $\mathbb{Q}$-basis in $V$, and by $\left\{f_{k}\right\}$ the dual basis. Let $A$ and $B$ be operators acting on $V \otimes_{\mathbb{Q}} \mathbb{C}$. Define

$$
\left\langle v_{0}\left|A \otimes_{\mathbb{Q}} B\right| f_{n}\right\rangle:=\sum_{v_{k}}\left\langle v_{0}|A| f_{k}\right\rangle \otimes_{\mathbb{Q}}\left\langle v_{k}|B| f_{n}\right\rangle \in \mathbb{C} \otimes_{\mathbb{Q}} \mathbb{C}
$$

where the sum is over all basis vectors $v_{k}$. Since we take the tensor product over $\mathbb{Q}$, this expression is independent of the choice of a basis over $\mathbb{Q}$. (It might be different for a basis over $\mathbb{C}$.)

Let $\left(H ; v_{0}, f_{n}\right)$ be a splitted framed Hodge-Tate structure, and let $\mathcal{M}$ be the period operator on $\bigoplus_{k} g r_{-2 k}^{W} H_{\mathbb{Q}}$. We define the big period as

$$
P_{n}^{\prime}\left(\left(H ; v_{0}, f_{n}\right)\right):=\left\langle v_{0}\left|\mathcal{M}^{-1} \otimes_{\mathbb{Q}} \mathcal{M}\right| f_{n}\right\rangle \in \mathbb{C} \otimes \mathbb{C} .
$$

One can rewrite it as

$$
\left\langle v_{0}\left|\sum_{k=0}^{\infty}(1-\mathcal{M})^{k} \otimes_{\mathbb{Q}} \mathcal{M}\right| f_{n}\right\rangle .
$$

Lemma 4.2. The big period does not depend on the choice of splitting.

Proof. The period matrix corresponding to a different splitting is $\mathcal{N M}$, where $\mathcal{N}$ is a (unipotent upper triangular) matrix with rational entries. One has

$$
\left\langle v_{0}\left|(\mathcal{N} \mathcal{M})^{-1} \otimes_{\mathbb{Q}} \mathcal{N} \mathcal{M}\right| f_{n}\right\rangle=\left\langle v_{0}\left|\mathcal{M}^{-1} \otimes_{\mathbb{Q}} \mathcal{M}\right| f_{n}\right\rangle
$$

Recall that a variation of mixed Hodge structures is supposed to satisfy the Griffith transversality condition. Let $\mathcal{M}$ be a period matrix of the variation of splitted framed Hodge-Tate structures.

Proposition 4.3. The Griffith transversality condition implies the following differential equations on the entries of the period matrix $\mathcal{M}$ :

$$
\sum_{k=0}^{\infty}\left\langle v_{0}\left|(1-\mathcal{M})^{k} \cdot d \mathcal{M}\right| f_{n}\right\rangle=\left\langle v_{0}\left|\mathcal{M}^{-1} d \mathcal{M}\right| f_{n}\right\rangle=0 .
$$

The proof of this proposition is given in s. 4.6 below.

4. The big period map via the Hopf algebra $\tilde{\mathcal{H}}_{\text {. }}$ Let $A$ be a Hopf algebra over $\mathbb{Q}$ with a product $\mu: A \otimes A \longrightarrow A$ and a coproduct $\nu: A \longrightarrow A \otimes A$. Then there are maps

$$
\mu^{(n)}: \otimes^{n} A \longrightarrow A ; \quad \nu^{(n)}: A \longrightarrow \otimes^{n} A
$$

Namely, $\mu^{(n)}: a_{1} \otimes \ldots \otimes a_{n} \longmapsto a_{1} \cdot \ldots \cdot a_{n}$. Here we have to specify the order of multiplication by putting brackets; however, thanks to the associativity of the product map the result is independent of this. Dualizing we get the canonical map $\nu^{(n)}$. For example one can set

$$
\nu^{(n)}:=(I d \otimes \ldots \otimes I d \otimes \nu) \circ \ldots \circ(I d \otimes \nu) \circ \nu .
$$

The map $\nu^{(n)}$ is a morphism of algebras since $A$ is a Hopf algebra.

Let $\tilde{A}$ be the kernel of the counit map $\hat{e}: A \rightarrow \mathbb{Q}$. Then $A=\mathbb{Q} \oplus \tilde{A}$. Projecting the coproduct to $\tilde{A} \otimes \tilde{A}$ we get the reduced coproduct map

$$
\tilde{\nu}: A \longrightarrow \tilde{A} \otimes \tilde{A},
$$


and similarly maps $\tilde{\nu}^{(n)}: A \longrightarrow \mathbb{Q}^{n} \tilde{A}$. Suppose $A=\bigoplus_{n>0} A_{n}, A_{0}=\mathbb{Q}$ is a graded Hopf algebra. Identifying $\tilde{A}=A_{>0}$ we get a map $\tilde{\nu}^{(n)}: A \longrightarrow \bigotimes^{n} A_{>0}$. Let us define for $n \geq 2$ a homomorphism of algebras $m_{n}: A \longrightarrow A_{>0} \otimes A_{>0}$ as a composition

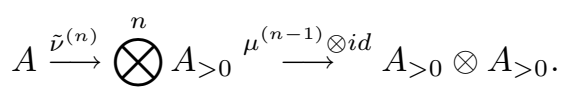

Set $m_{1}: A \longrightarrow A \otimes A, a \longmapsto 1 \otimes a$, and

$$
m: A \longrightarrow A \otimes A, \quad m:=\sum_{n \geq 1}^{\infty}(-1)^{n-1} m_{n}
$$

The sum is finite since $m_{n}\left(A_{k}\right)=0$ if $k>n$.

Let us define a map $m^{\prime}: A \longrightarrow A \otimes 1 \hookrightarrow A \otimes A$ by setting

$$
m^{\prime}:=\sum_{n \geq 1}^{\infty}(-1)^{n} \mu_{n} \circ \nu_{n}: A \longrightarrow A=A \otimes 1 \hookrightarrow A \otimes A, \quad \nu_{1}=\mu_{1}=\text { id. }
$$

Let us apply this construction to the Hopf algebra $\tilde{\mathcal{H}}_{\bullet}$. Notice that we have $\tilde{\nu}:=\nu-1 \otimes \mathrm{id}-\mathrm{id} \otimes 1$. We are gettting a map

$$
m_{\mathcal{H}}+m_{\mathcal{H}}^{\prime}: \tilde{\mathcal{H}}_{\bullet} \longrightarrow \tilde{\mathcal{H}}_{\bullet} \otimes \tilde{\mathcal{H}}_{\bullet}
$$

Lemma 4.4. The big period map $P_{n}^{\prime}$ is defined as a composition

$$
\tilde{\mathcal{H}}_{\bullet} \stackrel{m_{\mathcal{H}}+m_{\mathcal{H}}^{\prime}}{\longrightarrow} \tilde{\mathcal{H}}_{\bullet} \otimes \tilde{\mathcal{H}}_{\bullet} \stackrel{\tilde{p} \otimes \tilde{p}}{\longrightarrow} \mathbb{C} \otimes_{\mathbb{Q}} \mathbb{C} .
$$

Proof. Using formula (41) we can write the big period of a splitted Hodge-Tate structure $H$ as

$$
1 \otimes \tilde{p}\left(v_{0}, f_{n}\right)
$$

$$
\begin{gathered}
+\sum_{k \geq 2} \sum_{0<i_{1}<\ldots<i_{k-1}<n}(-1)^{k-1} \tilde{p}\left(v_{0}, f_{i_{1}}\right) \cdot \ldots \cdot \tilde{p}\left(v_{i_{-k+2}}, f_{i_{k-1}}\right) \otimes \tilde{p}\left(v_{i_{k-1}}, f_{n}\right) \\
+\sum_{k \geq 1} \sum_{0<i_{1}<\ldots<i_{k-1}<n}(-1)^{k} \tilde{p}\left(v_{0}, f_{i_{1}}\right) \cdot \ldots \cdot \tilde{p}\left(v_{i_{k-1}}, f_{n}\right) \otimes 1
\end{gathered}
$$

The sum is over all nonempty chains of basis vectors $v_{i} \in g r_{-2 i}^{W} H_{\mathbb{Q}}, 0<i<n$, and $f_{i}$ is the vector of the dual basis corresponding to $v_{i}$, so $\left\langle f_{i}, v_{i}\right\rangle=1$. The lemma follows.

Let $P_{n}$ be the composition of $P_{n}^{\prime}$ with the map

$$
\mathbb{C} \otimes_{\mathbb{Q}} \mathbb{C} \longrightarrow \mathbb{C} \otimes_{\mathbb{Q}} \mathbb{C}^{*}(n-2), \quad a \otimes b \longmapsto 2 \pi i \cdot a \otimes \exp (2 \pi i \cdot b) \otimes(2 \pi i)^{n-2} .
$$

Then $P_{n}$ is the composition

$$
\tilde{\mathcal{H}}_{\bullet} \quad \stackrel{m_{\mathcal{H}}}{\longrightarrow} \tilde{\mathcal{H}}_{\bullet} \otimes \tilde{\mathcal{H}}_{\bullet} \stackrel{\tilde{p} \otimes \tilde{p}}{\longrightarrow} \mathbb{C} \otimes \mathbb{Q} \mathbb{C} \longrightarrow \mathbb{C} \otimes \mathbb{Q} \mathbb{C}^{*}(n-2) .
$$

The term corresponding to $m_{\mathcal{H}}^{\prime}$ (i.e. the term (44)) disappears after the projection $\mathbb{C} \otimes_{\mathbb{Q}} \mathbb{C} \longrightarrow \mathbb{C} \otimes_{\mathbb{Q}} \mathbb{C}^{*}(n-2)$.

Let $S$ be a regular complex variety. There is a map

$$
\omega: \mathcal{O}_{S} \otimes_{\mathbb{Q}} \mathcal{O}_{S} \longrightarrow \Omega_{S}^{1}, \quad f \otimes g \longmapsto f d g .
$$


Theorem 4.5. a) The map $P_{n}^{\prime}$ provides a homomorphism $\mathcal{H}_{n} \longrightarrow \mathbb{C} \otimes_{\mathbb{Q}} \mathbb{C}$.

b) The restriction of the map $P_{n}$ to $\operatorname{Ker}\left(\tilde{\nu}_{n}\right)$ coincides with the natural isomorphism

$$
\frac{\mathbb{C}}{(2 \pi i)^{n} \mathbb{Q}}=2 \pi i \otimes \mathbb{C}_{\mathbb{Q}}^{*}(n-2) .
$$

c) Let $H_{S}$ be a variation of $n$-framed mixed Hodge-Tate structures over a base $S$. Then the big period map provides us with a section $P_{n}^{\prime}\left(H_{S}\right) \in \mathcal{O}_{S} \otimes_{\mathbb{Q}} \mathcal{O}_{S}$, and the composition $H_{S} \stackrel{P_{n}^{\prime}}{\longrightarrow} \mathcal{O}_{S} \otimes \mathcal{O}_{S} \stackrel{\omega}{\longrightarrow} \Omega_{S}^{1}$ is zero.

Proof. a) Follows from Lemma 4.4.

b) Clear from the definitions.

c) We will prove it in s. 4.6 below.

5. Examples. Let us define a Hodge-Tate structure $M$ using the following period matrix $\mathcal{M}$, where $x_{i}, y_{j}, z_{1}$ are arbitrary complex numbers:

$$
\mathcal{M}:=\left(\begin{array}{cccc}
1 & x_{1} & x_{2} & x_{3} \\
0 & 1 & y_{1} & y_{2} \\
0 & 0 & 1 & z_{1} \\
0 & 0 & 0 & 1
\end{array}\right)
$$

Denote by $\mathcal{I}$ the matrix of the operator acting by $(2 \pi i)^{-k}$ on $g r_{2 k}^{W} H_{\mathbb{Q}}$. Set $\tilde{\mathcal{M}}=$ $\mathcal{M I}$. We call $\tilde{\mathcal{M}}$ the canonical period matrix. So

$$
\tilde{\mathcal{M}}:=\left(\begin{array}{cccc}
1 & \tilde{x}_{1} & \tilde{x}_{2} & \tilde{x}_{3} \\
0 & 2 \pi i & 2 \pi i \cdot \tilde{y}_{1} & 2 \pi i \cdot \tilde{y}_{2} \\
0 & 0 & (2 \pi i)^{2} & (2 \pi i)^{2} \cdot \tilde{z}_{1} \\
0 & 0 & 0 & (2 \pi i)^{3}
\end{array}\right)
$$

where $\tilde{x}_{k}:=(2 \pi i)^{k} \cdot x_{k}, \tilde{y}_{j}:=(2 \pi i)^{j} \cdot y_{j}, \tilde{z}_{1}:=2 \pi i \cdot z_{1}$.

Let $C_{i}$ be the $i$-th row of the matrix $\tilde{\mathcal{M}}$, and let $e_{j}$ be the row whose only nonzero entry is 1 in the $j$-th place, $i, j=0,1,2,3$. We define the weight filtration by

$$
\begin{gathered}
W_{-6} M:=\left\langle C_{3}\right\rangle_{\mathbb{Q}}, \quad W_{-4} M:=\left\langle C_{2}, C_{3}\right\rangle_{\mathbb{Q}}, \quad W_{-2} M:=\left\langle C_{1}, C_{2}, C_{3}\right\rangle_{\mathbb{Q}}, \\
W_{0} M:=\left\langle C_{0}, C_{1}, C_{2}, C_{3}\right\rangle_{\mathbb{Q}}
\end{gathered}
$$

and the Hodge filtration by

$$
\begin{gathered}
F^{0} M:=\left\langle e_{0}\right\rangle, \quad F^{-1} M:=\left\langle e_{0}, e_{-1}\right\rangle, \quad F^{-2} M:=\left\langle e_{0}, e_{-1}, e_{-2}\right\rangle, \\
F^{-3} M:=\left\langle e_{0}, e_{-1}, e_{-2}, e_{-3}\right\rangle .
\end{gathered}
$$

The Hodge-Tate structure $M$ is equipped with a canonical framing given by $e_{0}$ and $(2 \pi i)^{3} e_{3}$. The corresponding period is $x_{3}=(2 \pi i)^{-3} \tilde{x}_{3}$. The big period is

$$
\begin{gathered}
P_{3}^{\prime}(M)=1 \otimes x_{3}+\left(-x_{1}\right) \otimes y_{2} \\
+\quad\left(-x_{2}+x_{1} y_{1}\right) \otimes z_{1}+\left(-x_{3}+x_{1} y_{2}+x_{2} z_{1}-x_{1} y_{1} z_{1}\right) \otimes 1 \in \mathbb{C} \otimes \mathbb{C} .
\end{gathered}
$$

The following is a classical example of the period matrix for the variation of Hodge-Tate structures related to the dilogarithm (due to Deligne):

$$
\tilde{\mathcal{L}}_{2}:=\quad\left(\begin{array}{ccc}
1 & -L i_{1}(z) & -L i_{2}(z) \\
0 & 2 \pi i & 2 \pi i \cdot \log z \\
0 & 0 & (2 \pi i)^{2}
\end{array}\right) .
$$


Then

$$
P_{2}\left(\mathcal{L}_{2}\right)=2 \pi i \otimes \exp \left(\frac{-L i_{2}(z)}{2 \pi i}\right)-\log (1-z) \otimes z
$$

It was first written by S. Bloch [B13]. Generalizing it, $S y m_{\mathbb{Q}}^{n-1} \mathbb{C} \otimes \mathbb{C}^{*}$-valued invariants of the Hodge-Tate structures related to classical $n$-logarithms were constructed in [BD] and [Bl4]. However the approach of these papers is quite different; it uses a very specific structure of the Hodge-Tate structures related to classical polylogarithms, and so cannot be generalized to other mixed Tate motives.

Remark. The entries of the canonical period matrix $\tilde{\mathcal{M}}$ for the Hodge realization of a mixed Tate motive $M$ are periods of rational algebraic differential forms over relative cycles. More precisely $\tilde{\mathcal{M}}$ is the matrix of the comparision isomorphism $M_{D R} \otimes \mathbb{C} \longrightarrow M_{\text {Betti }} \otimes \mathbb{C}$ in the natural $\mathbb{Q}$-bases in $M_{D R}$ and $M_{\text {Betti }}$.

6. The differential equations on the periods and the Griffith transversality condition. Let $\tilde{\mathcal{H}}(U)$ be the Hopf algebra of the splitted framed analytic variations of the Hodge-Tate structures over an analytic domain $U$. Define a homomorphism $\Omega_{n}: \tilde{\mathcal{H}}(U)_{n} \longrightarrow \Omega_{U}^{1}$ as the composition

$$
\tilde{\mathcal{H}}(U)_{n} \stackrel{\nu_{n-1,1}}{\longrightarrow} \tilde{\mathcal{H}}(U)_{n-1} \otimes \tilde{\mathcal{H}}(U)_{1} \stackrel{\tilde{p} d \tilde{p}}{\longrightarrow} \Omega_{U}^{1} .
$$

Say that a partial period $\tilde{p}\left(\tilde{H} ; v_{k}, f_{l}\right)$, where $v_{k} \in g r_{-2 k}^{W} \tilde{H}$ and $f_{l} \in\left(g r_{-2 l}^{W} \tilde{H}\right)^{*}$, has amplitude $l-k$.

Lemma 4.6. a) The maximal period of a variation of splitted Hodge-Tate structures $\tilde{H}_{U}$ over a domain $U$ satisfies the differential equation

$$
d \tilde{p}\left(\tilde{H}_{U} ; v_{0}, f_{n}\right)=\Omega_{n}\left(\tilde{H}_{U} ; v_{0}, f_{n}\right) .
$$

b) The Griffith transversality condition is equivalent to the differential equations (48) for all the partial periods of amplitudes $\geq 2$ for $\tilde{H}_{U}$.

In terms of the period matrix the differential equation (48) can be written as

$$
d \tilde{p}\left(\tilde{H}_{U} ; v_{0}, f_{n}\right)=\sum_{j} \tilde{p}\left(\tilde{H}_{U} ; v_{0}, f_{n-1}^{j}\right) d \tilde{p}\left(\tilde{H}_{U} ; v_{n-1}^{j}, f_{n}\right)
$$

where the sum is over a basis $\left\{v_{n-1}^{j}\right\}$ of $g r_{-2(n-1)}^{W} \tilde{H}_{U}$.

Proof. Let us present a detailed calculation for the variation of the Hodge-Tate structure described by the matrix $\mathcal{M}$ in the example above. The general case is completely similar, but requires more elaborate notation. Since the columns $C_{i}$ are horizontal, one has the equations

$$
\begin{gathered}
\nabla\left(e_{3}\right)=0, \quad \nabla\left(e_{2}+z_{1} e_{3}\right)=0, \quad \nabla\left(e_{1}+y_{1} e_{2}+z_{1} e_{3}\right)=0, \\
\nabla\left(e_{0}+x_{1} e_{1}+y_{1} e_{2}+z_{1} e_{3}\right)=0 .
\end{gathered}
$$

Solving them step by step we get

$$
\nabla\left(e_{3}\right)=0, \quad \nabla e_{2}=-d z_{1} e_{3}, \quad \nabla e_{1}=-d y_{1} e_{2}, \quad d x_{2}=x_{1} d y_{1}, \quad \nabla e_{0}=-d x_{1} e_{1} .
$$

So there is only one differential equation in this case: $d x_{2}=x_{1} d y_{1}$.

Proof of part c) of Theorem 4.5. Let $\otimes \mathcal{H}_{\bullet}:=\bigoplus_{k=1}^{\infty} \otimes^{k} \mathcal{H}_{\bullet}$. Define an operator $D: \otimes \mathcal{H}_{\bullet} \longrightarrow \otimes \mathcal{H}_{\bullet}$ as follows. Its restriction to $\mathcal{H}_{n}$ is $\nu_{n-1,1}$ if $n>1$ and the identity map if $n=1$. In general we set

$$
D\left(a_{n_{1}} \otimes \ldots \otimes a_{n_{k}}\right):=a_{n_{1}} \otimes \ldots \otimes a_{n_{k}} \otimes D\left(a_{n_{k}}\right) .
$$


It is easy to see that $D \circ\left(\sum_{k \geq 1}(-1)^{k-1} \tilde{\nu}^{(k)}\right)=0$. By formulas (48) and Lemma 4.4 the map $(2 \pi i)^{-2} \cdot \omega \circ P_{n}^{\prime}: \mathcal{H}_{n} \longrightarrow \Omega_{U}^{1}$ is the composition

$$
\mathcal{H}_{n} \stackrel{\sum_{k \geq 1}(-1)^{k-1} \tilde{\nu}^{(k)}}{\longrightarrow} \bigotimes \mathcal{H}_{\bullet} \stackrel{D}{\longrightarrow} \bigotimes \mathcal{H}_{\bullet} \stackrel{\mu \otimes \mathrm{id}}{\longrightarrow} \mathcal{H}_{\bullet} \otimes \mathcal{H}_{\bullet} \stackrel{\tilde{p} d \tilde{p}}{\longrightarrow} \Omega_{U}^{1} .
$$

Here $\mu$ is the product of all but the last components of $\otimes \mathcal{H}_{\bullet}$. The statement follows.

Example. For the Hodge-Tate structure $M$ from the example above (see (47)) we have

$$
(2 \pi i)^{-2} \cdot \omega \circ P_{3}^{\prime}(M)=d x_{3}-x_{1} d y_{2}-x_{2} d z_{1}+x_{1} y_{1} d z_{1}=0
$$

since $d x_{3}=x_{2} d z_{1}$ and $d y_{2}=y_{1} d z_{1}$.

7. The hyperbolic volumes are the real periods of the corresponding mixed Hodge structures. Recall the mixed Hodge structure

$$
h(Q ; M)(n):=\quad H^{2 n-1}\left(\mathbb{C P}^{2 n-1} \backslash Q ; M \backslash Q \cap M, \mathbb{Q}\right)(n) .
$$

Theorem 4.7. Suppose that the pair $(Q, M)$ corresponds to a hyperbolic geodesic simplex. Then the real period of the Hodge-Tate structure $h(Q, M)(n)$ equals the volume of the corresponding geodesic simplex.

Proof. The mixed Hodge structure $h(Q, M)(n)$ has a natural splitting of its weight filtration such that its maximal period $\tilde{p}_{\alpha}(Q, M)$ is given by

$$
\tilde{p}_{\alpha}(Q, M)=\int_{\Delta_{M}} \omega_{Q}
$$

where the sign of the form $\omega_{Q}$ is determined by the family $\alpha$ of maximal isotropic planes on $Q$. In fact using the definition of a period from s. 4.1 we need only a canonical choice of a cycle $\Delta_{M}$, which is provided by the geodesic simplex. Then

$$
p_{\alpha}(Q, M):=\frac{\tilde{p}_{\alpha}(Q, M)}{(2 \pi i)^{n}} \in \begin{cases}\mathbb{R} & \text { if }(Q, M) \text { is spherical, } \\ i \mathbb{R} & \text { if }(Q, M) \text { is hyperbolic. }\end{cases}
$$

Therefore $\log \left|\exp \left(2 \pi i \cdot p_{\alpha}(Q, M)\right)\right|=1$ if $(Q, M)$ is spherical. So using Lemma 4.4 for the computation of the big period map together with the following crucial fact:

$$
\nu^{(j)} h(Q, M)(n) \in \quad \mathcal{P}\left(\mathcal{H}^{2 k_{1}-1}\right) \otimes \mathcal{P}\left(S^{2 k_{2}-1}\right) \otimes \ldots \otimes \mathcal{P}\left(S^{2 k_{j}-1}\right)
$$

we conclude that $2 \pi i \otimes \exp \left(2 \pi i \cdot p_{\alpha}(Q, M)\right) \otimes(2 \pi i)^{n-2}$ is the only component of the big period of $h(Q, M)$ whose contribution to the real period is different from zero. The theorem is proved.

It seems that in general there is no similar interpretation of the spherical volumes. However the restriction of the volume homomorphism

$$
\text { vol : } \operatorname{Ker} D_{2 n-1}^{S} \longrightarrow i^{n} \mathbb{R} /(2 \pi i)^{n} \mathbb{Q}
$$

can be identified with the Hodge realization map

$$
\text { Hod : } K e r D_{2 n-1}^{S} \longrightarrow K e r \tilde{\nu}_{n}=\mathbb{C} /(2 \pi i)^{n} \mathbb{Q}
$$

provided by the map $(Q, M) \longmapsto h(Q, M)(n)$. Notice that $\operatorname{Hod}\left(\operatorname{Ker} D_{2 n-1}^{S}\right) \subset$ $\operatorname{Ker} \tilde{\nu}_{n}$ by Theorem 1.5. Further, if $\tilde{\nu}_{n}(\operatorname{Hod}(S))=0$, then in the formula for $P_{n}(\operatorname{Hod}(S))$ the only nonzero term is $(2 \pi i)^{n-2} \otimes 2 \pi i \otimes \exp (2 \pi i \cdot \operatorname{vol}(S))$. So after the identification $(46)$ we get $\operatorname{vol}(S)$ modulo $(2 \pi i)^{n} \mathbb{Q}$. 
8. The Schläfli formula. Applying formula (48) to the variation of Hodge-Tate structures provided by a family of configurations $(Q, M)$ we get the differential equation on the periods $p_{\alpha}(Q, M)$. Let us write it explicitly. There are exactly 2 different families $\alpha$ and $\beta$ of maximal isotropic subspaces on an even-dimensional smooth quadric $Q$. For each isotropic subspace $\gamma$ of codimension two there are just 2 maximal isotropic subspaces $\alpha_{(\gamma)}$ and $\beta_{(\gamma)}$ containing it. They belong to families $\alpha$ and $\beta$ respectively. Let $H$ be a generic codimension 2 hyperplane. Choose an isotropic subspace $\gamma \subset H \cap Q$. Then there are just two hyperplanes $H_{\gamma}^{\alpha}$ and $H_{\gamma}^{\beta}$ containing $H$ and tangent to the quadric $Q$ (here $\alpha_{(\gamma)} \subset H_{\gamma}^{\alpha}$ ).

Set $M_{i j}=M_{i} \cap M_{j}$. Choose a maximal isotropic subspace $\gamma_{i j} \subset M_{i j} \cap Q$. Let $H_{\gamma_{i j}}^{\alpha}$ and $H_{\gamma_{i j}}^{\beta}$ be the corresponding hyperplanes tangent to $Q$ and containing $M_{i j}$. The hyperplanes containing $M_{i j}$ form a projective line. Let $r\left(M_{i}, M_{j}, H_{\gamma_{i j}}^{\alpha}, H_{\gamma_{i j}}^{\beta}\right)$ be the cross-ratio of 4 points on it corresponding to the hyperplanes.

Set $Q_{i j}:=Q \cap M_{i j}$. The hyperplanes $M_{k}, k \neq i, j$, cut a simplex $M^{i j}$ in $M_{i j}$. The Schläfli formula is the following differential equation:

$$
d p_{\alpha}(Q, M)=\sum_{i<j} p_{\gamma_{i j}}\left(Q_{i j}, M^{i j}\right) \cdot d \log r\left(M_{i}, M_{j}, H_{\gamma_{i j}}^{\alpha}, H_{\gamma_{i j}}^{\beta}\right) .
$$

Notice that if $\gamma_{i j}^{\prime}$ is a maximal isotropic subspace from a different family, then both the cross-ratio and period $v$ change sign.

This formula implies (using Theorem 5.3 in [G3]) that the integrals $p_{\alpha}(Q, M)$ can be expressed via the hyperlogarithms of order $n$. Since the hyperlogarithms of order 3 can be expressed by the classical trilogarithms and products of dilogarithm and logarithms (see [G3]) we come to the expression of integrals $p_{\alpha}(Q, M)$ by means of classical trilogarithms and products of dilogarithm and logarithms. See [K1] for another method of proving this result.

\section{Mixed Tate motives over a number field And Theorem 1.6}

1. The category of mixed Tate motives over $F$. According to Beilinson there should exist a category $\mathcal{M}(F)$ of mixed motives over a field $F$ with the following properties (see [B1]; we present only a part of the list needed for our purposes).

It is tensor $\mathbb{Q}$-category with a fixed invertible object $\mathbb{Q}(1)$. There are simple objects $\mathbb{Q}(m):=\mathbb{Q}(1)^{\otimes m}, m \in \mathbb{Z}$, and these objects are mutually nonisomorphic.

$$
\begin{gathered}
\operatorname{Ext}_{\mathcal{M}(F)}^{1}(\mathbb{Q}(0), \mathbb{Q}(m))=0 \text { for } m \leq 0, \\
\operatorname{Ext}_{\mathcal{M}(F)}^{i}(\mathbb{Q}(0), \mathbb{Q}(n))=g r_{n}^{\gamma} K_{2 n-i}(F)_{\mathbb{Q}} .
\end{gathered}
$$

Any object carries a canonical increasing finite filtration (weight filtration), which is strictly compatible with the morphisms.

The category $\mathcal{M}_{T}(F)$ of mixed Tate motives over $F$ is a full tensor subcategory generated by the Tate object $\mathbb{Q}(1)$ and closed under extensions. Any simple object in $\mathcal{M}_{T}(F)$ is isomorphic to $\mathbb{Q}(n)$.

In the category $M H S$ of the mixed $\mathbb{Q}$-Hodge structures one has

$$
\operatorname{Ext}_{M H S}^{1}(\mathbb{Q}(0), \mathbb{Q}(n))=\mathbb{C} /(2 \pi i)^{n} \mathbb{Q} .
$$

Thus the realization functor provides a homomorphism

$$
g r_{n}^{\gamma} K_{2 n-1}(\mathbb{C})_{\mathbb{Q}}=\operatorname{Ext}_{\mathcal{M}(\mathbb{C})}^{1}(\mathbb{Q}(0), \mathbb{Q}(n)) \longrightarrow \operatorname{Ext}_{M H S}^{1}(\mathbb{Q}(0), \mathbb{Q}(n))=\mathbb{C} /(2 \pi i)^{n} \mathbb{Q}
$$

that should coincide with Beilinson's regulator. 
In complete analogy with the case of Hodge-Tate structures one can define $n$ framed mixed Tate motives over $F$ ([BMS], see Section 3 of [G4] for details). Their equivalence classes form an abelian group denoted $\mathcal{A}_{n}(F)$, and $\mathcal{A}_{\bullet}(F):=$ $\bigoplus_{n \geq 0} \mathcal{A}_{n}(F)$ is equipped with a structure of a commutative graded Hopf algebra. The category of mixed Tate motives is canonically equivalent to the category of finite-dimensional graded $\mathcal{A}_{\bullet}(F)$-modules. One has a canonical morphism

$$
H_{(n)}^{i}\left(\mathcal{A}_{\bullet}(F), \mathbb{Q}\right)=\operatorname{Ext}_{\mathcal{M}_{T}(F)}^{i}(\mathbb{Q}(0), \mathbb{Q}(n)) \longrightarrow \operatorname{Ext}_{\mathcal{M}(F)}^{i}(\mathbb{Q}(0), \mathbb{Q}(n)) .
$$

Here the group on the left is the degree $n$ part of the graded vector space $H^{i}\left(\mathcal{A}_{\bullet}(F), \mathbb{Q}\right)$. Composing with $(51)$ we get a map

$$
H_{(n)}^{i}\left(\mathcal{A}_{\bullet}(F), \mathbb{Q}\right) \longrightarrow g r_{n}^{\gamma} K_{2 n-i}(F) \otimes \mathbb{Q} .
$$

Remark. It was also conjectured by Beilinson that the Ext groups between $\mathbb{Q}(0)$ and $\mathbb{Q}(n)$ in the category of mixed Tate motives are the same as in the category of all mixed motives. This would imply that maps (53) and (54) are isomorphisms.

The Hopf algebra $\mathcal{A}_{\bullet}(F)$ is the dual to the universal enveloping algebra of the following graded Lie algebra $\left(V^{\vee}\right.$ is the duality between the ind and pro $\mathbb{Q}$-vector spaces):

$$
L \bullet(F):=\left(\frac{\mathcal{A}_{>0}(F)}{\left(\mathcal{A}_{>0}(F)\right)^{2}}\right)^{\vee} .
$$

2. Implications for the Dehn complexes. Let $F$ be an algebraically closed field. Each generator of $S_{n}(F)$ defines an $n$-framed Hodge-Tate structure realised in the cohomology of a (simplicial) algebraic variety and therefore should define an $n$-framed mixed Tate motive. This leads to a canonical homomorphism of graded Hopf algebras

$$
S(F) \bullet \longrightarrow \mathcal{A}_{\bullet}(F)
$$

Using (54) we get canonical homomorphisms

$$
H_{(n)}^{i}(S(F) \bullet, \mathbb{Q}) \longrightarrow g r_{n}^{\gamma} K_{2 n-i}(F) \otimes \mathbb{Q} .
$$

One can compute the left side as the cohomology of the degree $n$ part of the cobar complex $S(F ; n)^{\bullet}$ for the graded Hopf algebra $S(F) \bullet$ :

$$
S_{n}(F) \longrightarrow \bigoplus_{i_{1}+i_{2}=n} S_{i_{1}}(F) \otimes S_{i_{2}}(F) \longrightarrow \bigoplus_{i_{1}+i_{2}+i_{3}=n} S_{i_{1}}(F) \otimes S_{i_{2}}(F) \otimes S_{i_{3}}(F) \longrightarrow \ldots
$$

Here the left group is placed at degree $1\left(i_{k}>0\right)$, and the coboundary is of degree +1 . Therefore we get part a) of the following conjecture.

Conjecture 5.1. a) There is a canonical homomorphism

$$
H^{i}\left(S(F ; n)^{\bullet}\right) \otimes \mathbb{Q} \longrightarrow g r_{n}^{\gamma} K_{2 n-i}(F) \otimes \mathbb{Q}
$$

b) It is injective for $i=1$.

c) The homomorphism (57) is an isomorphism. Moreover, (55) is an isomorphism of commutative graded Hopf algebras over $\mathbb{Q}$.

The second statement in c) is equivalent to the first. Of course c) implies b). We separate them since c) is much stronger and less motivated than b). 
Remark. An attempt to construct explicitly the Hopf algebra $\mathcal{A}_{\bullet}(F)$ was made in [BMS], [BGSV] using the scissor congruence groups of pairs of simplices in $\mathbb{P}^{n}$. However in that construction we still don't have a correct definition of the coproduct for degenerate configurations, while the product of two elements in that algebra is always degenerate. Strangely enough our construction of the Hopf algebra $S_{\bullet}(F)$ is free of this problem.

Notice that (56) is an isomorphism if and only if (55) is an isomorphism. I believe that (55) is at least injective; this provides part b) of Conjecture 5.1.

Let me deduce Conjecture 1.8 from Conjecture 5.1. Let us represent $\mathbb{C P}^{n}$ as a complexification of $\mathbb{R P}^{n}$. Then the complex conjugation acts on $\mathbb{C P}^{n}$.

Theorem 5.2. a) There are canonical homomorphisms

$$
\begin{aligned}
& \mathcal{P}\left(\mathcal{H}^{2 n-1}\right) \stackrel{\psi_{\mathcal{H}}(n)}{\longrightarrow}\left(S_{n}(\mathbb{C}) \otimes \mathbb{Z}(n)\right)^{-}, \\
& \mathcal{P}\left(S^{2 n-1}\right) \stackrel{\psi_{S}(n)}{\longrightarrow}\left(S_{n}(\mathbb{C}) \otimes \mathbb{Z}(n)\right)^{+} .
\end{aligned}
$$

b) They transform the Dehn invariant to the comultiplication, providing the following commutative diagram in the hyperbolic case:

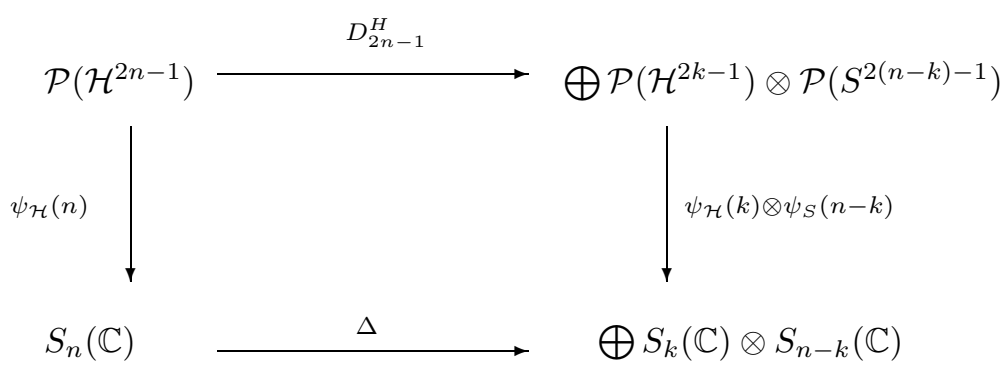

and a similar diagram in the spherical case.

c) The homomorphisms (58) , (59) provide the following homomorphisms from the hyperbolical and spherical Dehn complexes (see (9))

$$
\begin{aligned}
& \psi_{\mathcal{H}}^{\bullet}(n): \mathcal{P}_{\mathcal{H}}^{\bullet}(n) \longrightarrow\left(S(\mathbb{C} ; n)^{\bullet} \otimes \varepsilon(n)\right)^{-}, \\
& \psi_{S}^{\bullet}(n): \mathcal{P}_{S}^{\bullet}(n) \longrightarrow(S(\mathbb{C} ; n) \bullet \otimes \varepsilon(n))^{+} .
\end{aligned}
$$

Proof. The proof follows from the definition of $\Delta$ and Theorem 3.9a). The action of the complex conjugation on the image of scissors congruence groups is computed easily looking on the frames.

Combining these homomorphisms with Conjectures 5.1a) and 5.1b) we get Conjecture 1.7 and Conjecture 1.8a) respectively.

Remark. The homomorphisms $\psi_{\mathcal{H}}^{\bullet}(n), n>2$, and $\psi_{S}^{\bullet}(n), n \geq 2$, are not isomorphisms.

Using Theorems 3.11 and 5.2 we get Theorem 1.5.

3. Some results on $t$-structures on a triangulated category. Many results of this subsection are borrowed from $[\mathrm{BBD}]$ or $[\mathrm{P}]$. Recall that a $t$-structure on a triangulated category $\mathcal{D}$ is a pair of subcategories $\mathcal{D} \leq 0, \mathcal{D}^{\geq 1}$ satisfying the following conditions:

1) $\operatorname{Hom}_{\mathcal{D}}(X, Y)=0$ for all objects $X \in O b \mathcal{D} \leq 0$ and $Y \in \mathcal{D}^{\geq 1}$. 
2) For any $X \in O b \mathcal{D}$ there exists an exact triangle

$$
X_{\leq 0} \longrightarrow X \longrightarrow X_{\geq 1} \longrightarrow X_{\leq 0}[1]
$$

with $X_{\leq 0} \in \mathcal{D}^{\leq 0}$ and $X_{\geq 1} \in \mathcal{D}^{\geq 1}$.

3) $\mathcal{D}^{\leq 0} \subset \mathcal{D}^{\leq 1}$ and $\mathcal{D}^{\geq 1} \subset \mathcal{D}^{\leq 0}$.

Here $\mathcal{D}^{\leq a}:=\mathcal{D}^{\leq 0}[-a], \mathcal{D}^{\geq a}:=\mathcal{D}^{\geq 0}[-a], \mathcal{D}^{[a, b]}:=\mathcal{D}^{\leq b} \cap \mathcal{D}^{\geq a}$ and $\mathcal{D}^{a}:=\mathcal{D}^{[a, a]}$.

The exact triangle in 2) is defined uniquely up to an isomorphism and depends functorially on $X$. The subcategory $\mathcal{D}^{0}$ is called the heart of a $t$-structure. It is an abelian category $([\mathrm{BBD}])$.

Let $\mathcal{A}$ and $\mathcal{B}$ be two sets of isomorphism classes of objects in $\mathcal{D}$. Denote by $\mathcal{A} * \mathcal{B}$ the set of all objects $X$ in $O b \mathcal{D}$ which can be included into an exact triangle $A \longrightarrow X \longrightarrow B \longrightarrow A[1]$ with $A \in \mathcal{A}, B \in \mathcal{B}$.

Lemma 5.3. $(\mathcal{A} * \mathcal{B}) * \mathcal{C}=\mathcal{A} *(\mathcal{B} * \mathcal{C})$.

Proof. The proof follows from the octahedron lemma; see [BBD].

Theorem 5.4. a) Let $\mathcal{D}$ be a triangulated $\mathbb{Q}$-category, and let $\mathcal{Q}$ be a full semisimple subcategory generating $\mathcal{D}$ as a triangulated category. Suppose that for any two objects $Q_{1}, Q_{2} \in O b \mathcal{Q}$ one has

$$
\operatorname{Hom}_{\mathcal{D}}^{i}\left(Q_{1}, Q_{2}\right)=0, \quad i<0 .
$$

Then there exists a canonical t-structure on $\mathcal{D}$ with the abelian heart $\mathcal{M}=\bigcup \mathcal{Q} *$ $\mathcal{Q} * \ldots * \mathcal{Q}$.

b) If in addition $\operatorname{Hom}_{\mathcal{D}}^{i}\left(Q_{1}, Q_{2}\right)=0$ for $i \geq 2$, then the tensor category $\mathcal{D}$ is equivalent to the derived category of $\mathcal{M}$.

Theorem 5.5. Let $\mathcal{D}$ be a triangulated tensor $\mathbb{Q}$-category, and let $\mathcal{Q}$ be a full semisimple subcategory generated by nonisomorphic objects $\mathbb{Q}(m), m \in \mathbb{Z}$, such that $\mathbb{Q}(1)$ is invertible, $\mathbb{Q}(m)=\mathbb{Q}(1)^{\otimes m}$, and

$$
\operatorname{Hom}_{\mathcal{D}}^{i}(\mathbb{Q}(m), \mathbb{Q}(n))=0 \quad \text { if } m>n, \quad \operatorname{Hom}_{\mathcal{D}}(\mathbb{Q}(0), \mathbb{Q}(0))=\mathbb{Q} .
$$

Then the abelian heart $\mathcal{M}$ from Theorem 5.4 is a tensor category. It is equivalent to the tensor category of finite dimensional representations of a certain negatively graded (pro)-Lie algebra. This graded (pro)-Lie algebra is free if condition b) of Theorem 5.4 is satisfied.

Let $\mathcal{D}$ be a triangulated category, and let $\mathcal{M} \subset \mathcal{D}$ be a full subcategory.

Theorem 5.6. $\mathcal{M}$ is a heart for the unique bounded $t$-structure on $\mathcal{D}$ if and only if

i) $\mathcal{M}$ generates $\mathcal{D}$ as a triangulated category,

ii) $\mathcal{M}$ is closed with respect to extensions,

iii) $\operatorname{Hom}_{\mathcal{D}}(X, Y[i])=0$ for any $X, Y \in \mathcal{M}, \quad i<0$,

iv) $\mathcal{M} * \mathcal{M}[1] \subset \mathcal{M}[1] * \mathcal{M}$.

For the proof of this theorem see $[\mathrm{BBD}]$ or $[\mathrm{P}]$. Let me sketch the construction of the $t$-structure on $\mathcal{D}$. We will use the following

Lemma 5.7. Let

$$
X \longrightarrow Y \longrightarrow Z \stackrel{f}{\longrightarrow} X[1]
$$

be an exact triangle. Then $f=0$ if and only if $Y=X \oplus Z$. 
Proof. Let us show that $f=0$ implies $Y=X \oplus Z$. The composition of the identity morphism $Z \stackrel{\text { id }}{\longrightarrow} Z$ with $f$ is zero, so one has a morphism $g: Z \longrightarrow Y$ making the following diagram commutative:

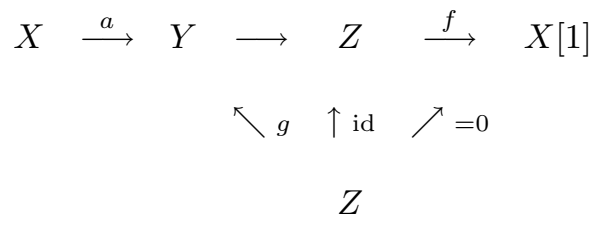

So there is a morphism $X \oplus Z \longrightarrow X$. The universality property of this morphism follows immediately from $f=0$.

Let $\mathcal{D}^{[a, b]}$ be the minimal full subcategory of $\mathcal{D}$ containing $\mathcal{M}[-i]$ for $a \leq i \leq b$ and closed under extensions. The subcategories $\mathcal{D} \leq 0$ and $\mathcal{D}^{\geq 1}$ are defined similarly and satisfy 1) thanks to iii). Notice that $\operatorname{Hom}_{\mathcal{D}}(\mathcal{M}, \mathcal{M}[-i])=0$ implies $\mathcal{M} *$ $\mathcal{M}[i+1]=\mathcal{M} \oplus \mathcal{M}[i+1]$ for $i>0$ by Lemma 5.7. So $\mathcal{M} * \mathcal{M}[n] \subset \mathcal{M}[n] * \mathcal{M}$ for any $n>0$. Using ii) and Lemma 5.3 we get $\mathcal{D}^{[a, b]}=\mathcal{M}[-a] * \mathcal{M}[-a-1] * \ldots * \mathcal{M}[-b]$. This proves 2). It remains to show that $\mathcal{D}^{\geq a} \cap \mathcal{D}^{\leq b} \subset \mathcal{D}^{[a, b]}$, and so in particular $\mathcal{D}^{0}=\mathcal{D}^{\geq 0} \cap \mathcal{D}^{\leq 0} \subset \mathcal{M}=\mathcal{D}^{[0,0]}$. If $X=Y_{c} * Y_{c+1} * \ldots * Y_{d} \in \mathcal{D}^{\geq a}$ where $Y_{i} \in \mathcal{M}[-i]$, then $\operatorname{Hom}\left(Y_{c}, X\right)=0$ for $c<a$ and so we get from the exact triangle

$$
Y_{c} \longrightarrow X \longrightarrow Y_{c+1} * \ldots * Y_{d} \longrightarrow Y_{c}[1]
$$

that $Y_{c+1} * \ldots * Y_{d}=X \oplus Y_{c}[1]$. Now $\operatorname{Hom}_{\mathcal{D}}\left(Y_{c}[1], Y_{c+1} * \ldots * Y_{d}\right)=0$ by iii) and so $Y_{c}=0$.

Proof of Theorem 5.4a). Let us use Theorem 5.6. The properties i)-ii) are obvious. iii) is easy to prove using (62), so we have to check only iv). Using the associativity of the $*$ operation we see that one has to prove only that $Q_{1} * Q_{2}[1] \subset Q_{2}[1] * Q_{1}$ for any two simple objects in $\mathcal{Q}$. One has

$$
Q_{1} \longrightarrow X \longrightarrow Q_{2}[1] \stackrel{f}{\longrightarrow} Q_{1}[1] .
$$

There are only two possibilities for $f$ :

1. $f$ is an isomorphism; then $X=0$.

2. $f=0$; then $X=Q_{1} \oplus Q_{2}[1]$ by Lemma 5.7.

Proof of Theorem 5.4b).

Proposition 5.8. a) Let $\mathcal{D}$ be a triangulated category, and let $\mathcal{M}$ be the heart of a t-structure on $\mathcal{D}$. Suppose that one has

$$
\operatorname{Hom}_{\mathcal{D}}^{i}(X, Y)=0 \quad \text { for any } \quad X, Y \in O b \mathcal{M}, \quad i>1 .
$$

Then the category $D^{b}(\mathcal{M})$ is equivalent to the category $\mathcal{D}$.

b) If we suppose in addition that the triangulated category $\mathcal{D}$ is a subcategory of a derived category $D^{b}(\mathcal{N})$ for some abelian category $\mathcal{N}$, then $D^{b}(\mathcal{M})$ is equivalent to $\mathcal{D}$ as a triangulated category.

Proof. Let $\tilde{\mathcal{D}}$ be the full subcategory of $\mathcal{D}$ whose objects are direct sums $\bigoplus A_{i}$ where $A_{i} \in \mathcal{D}^{-i}$, i.e. $H_{\mathcal{M}}^{j}\left(A_{i}\right)=0$ for $j \neq-i$. There is a canonical functor $i: \tilde{\mathcal{D}} \hookrightarrow \mathcal{D}$. Then (63) is a necessary and sufficient condition for $i$ to be an equivalence of categories. Indeed, let us show that every object $Y$ in $\mathcal{D}$ is isomorphic to $i(X)$ for 
some $X$. We may suppose $Y \in \mathcal{D}^{[0, n]}$ and will use induction by $n$. Consider the exact triangle

$$
H_{\mathcal{M}}^{0}(Y) \stackrel{f}{\longrightarrow} Y \longrightarrow C \longrightarrow H_{\mathcal{M}}^{0}(Y)[1]
$$

provided by the canonical morphism $f: H_{\mathcal{M}}^{0}(Y) \longrightarrow Y$. Then $C \in \mathcal{D}^{[1, n]}$ and $H_{\mathcal{M}}^{0}(Y)[1] \in \mathcal{D}^{-1}$, so it is easy to see that the morphism $C \longrightarrow H_{\mathcal{M}}^{0}(Y)[1]$ must equal 0 because $H_{o m} \geq 2$ are zero. Therefore $Y=H_{\mathcal{M}}^{0}(Y) \oplus C$ by the lemma above.

Let us show that $D^{b}(\mathcal{M})$ has cohomological dimension one, i.e.

$$
\operatorname{Hom}_{D^{b}(\mathcal{M})}^{i}(X, Y)=0 \quad \text { for any } \quad X, Y \in O b \mathcal{M}, \quad i>1 .
$$

Notice that for any $X, Y \in \mathcal{M}$ one has

$$
\operatorname{Hom}_{D^{b}(\mathcal{M})}^{i}(X, Y)=\operatorname{Ext}_{Y}^{i}(X, Y)
$$

where $\operatorname{Ext}_{Y}^{i}(X, Y)$ is the Yoneda $E x t$-groups in $\mathcal{M}$. Also

$$
\operatorname{Hom}_{\mathcal{D}}^{1}(X, Y)=\operatorname{Ext}_{Y}^{1}(X, Y) \text {. }
$$

Lemma 5.9. Let $\mathcal{D}$ be a triangulated category, and let $\mathcal{M}$ be the heart of a $t$ structure on $\mathcal{D}$. Then $\operatorname{Ext}_{Y}^{2}(X, Z) \subset \operatorname{Hom}_{\mathcal{D}}^{2}(X, Z)$.

Proof. Let us present an element of $\operatorname{Ext}_{Y}^{2}(X, Z)$ by $0 \longrightarrow Z \longrightarrow C_{2} \longrightarrow C_{1} \longrightarrow$ $X \longrightarrow 0$. Setting $Y:=\operatorname{Im}\left(C_{2} \longrightarrow C_{1}\right)$ we decompose it into a product of the following $E x t^{1}$ 's:

$$
0 \longrightarrow Z \longrightarrow C_{2} \longrightarrow Y \longrightarrow 0 \quad \text { and } \quad 0 \longrightarrow Y \longrightarrow C_{1} \longrightarrow X \longrightarrow 0 .
$$

Suppose that the composition of morphisms $X \rightarrow Y[1]$ and $Y[1] \rightarrow Z[2]$ in the triangulated category is zero. Then looking at the diagram

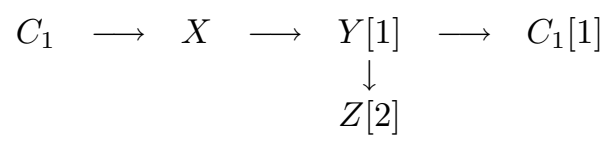

we conclude that there is a morphism $C_{1}[1] \rightarrow Z[2]$ making the diagram commutative. There is an object $W$ of the abelian category such that the exact sequence $0 \longrightarrow Z \longrightarrow W \longrightarrow C_{1} \longrightarrow 0$ represents the morphism $C_{1} \longrightarrow Z[1]$ in the triangulated category. So we get a diagram where the right square is commutative:

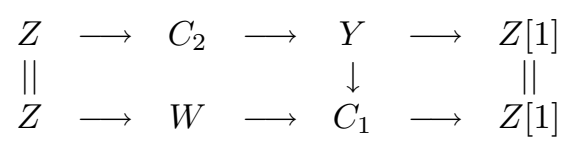

Therefore there exists a morphism $C_{2} \longrightarrow W$ making it commutative. So we get a commutative diagram

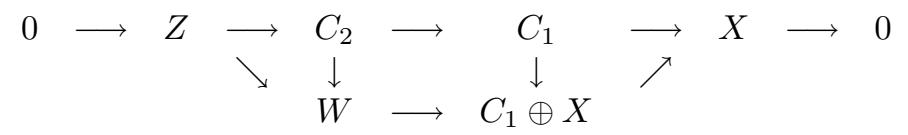

where the morphism $C_{1} \longrightarrow C_{1} \oplus X$ is given by $I d \oplus 0$. The $E x t_{Y}^{2}$ represented by the bottom sequence is zero. The lemma is proved.

Therefore $\operatorname{Hom}_{\mathcal{D}}^{2}(X, Y)=0$ implies $\operatorname{Ext}_{Y}^{2}(X, Y)=0$. Any element in $\operatorname{Ext}_{Y}^{n}$ can be represented as a product of certain elements from $E x t_{Y}^{1}$ and $E x t_{Y}^{n-1}$. So if $\operatorname{Ext}_{Y}^{2}(X, Y)=0$ for any 2 objects $X, Y$ in $\mathcal{M}$, then $\operatorname{Ext}_{Y}^{i}(X, Y)=0$ for all $i \geq 2$. So we have an equivalence of the categories. Part $b$ ) is proved using [BBD]. The proposition is proved. 
The proof of Theorem 5.5 is rather standard. One shows that $\mathcal{M}$ is a mixed Tate category (see [BD] or [G1], [G4] for the definitions) thanks to the conditions imposed on the Hom's between the $\mathbb{Q}(i)$ 's; then the Tannakian formalism leads to Theorem 5.5 (again see [BD] or [G1], [G4]).

4. The triangulated category of mixed Tate motives over $F$. I will work with the category $\mathcal{D M}_{F}$ of triangulated mixed motives over a field $F$ from [V]. An object in $\mathcal{D} \mathcal{M}_{F}$ is a "complex" of regular (but not necessarily projective) varieties $X_{1} \longrightarrow X_{2} \longrightarrow \ldots \longrightarrow X_{n}$ where the arrows are given by finite correspondences and the composition of any two successive arrows is zero. We denote by $M(X)$ the object of $\mathcal{D} \mathcal{M}_{F}$ corresponding to a smooth variety $X$ sitting in degree 0 . The Tate motive $\mathbb{Q}(1)$ is defined as the object $P^{1} \longrightarrow *$ of $\mathcal{D} \mathcal{M}_{F}$ sitting in degrees $[-2,-3]$, where $*$ is a point.

The objects $\mathbb{Q}(n) \in \mathcal{D} \mathcal{M}_{F}$ satisfy almost all the needed properties including the relation with K-theory:

$$
\operatorname{Ext}_{\mathcal{D} \mathcal{M}_{F}}^{i}(\mathbb{Q}(0), \mathbb{Q}(n))=g r_{n}^{\gamma} K_{2 n-i}(F) \otimes \mathbb{Q} .
$$

This formula follows from the results of Bloch, Suslin and Voevodsky; the key step is the relation of Higher Chow groups and algebraic K-theory proved by Bloch (see [Bl1] and the moving lemma in [Bl2]). For the relation between the Higher Chow groups and motivic cohomology (i.e. the left hand side in (65)) see [V], Proposition 4.2.9 (Higher Chow groups = Borel-Moore motivic homology), and [V], Proposition 4.3.7 (duality for smooth varieties).

We define the triangulated category of mixed Tate motives $\mathcal{D}_{T}(F)$ as the triangulated subcategory of $\mathcal{D} \mathcal{M}_{F}$ generated by the objects $\mathbb{Q}(i), i \in \mathbb{Z}$. The following proposition is due to Voevodsky (unpublished).

Proposition 5.10. A direct summand of any object of $\mathcal{D}_{T}(F)$ belongs to $\mathcal{D}_{T}(F)$.

Proof. Let $\mathcal{D}_{T}(\geq 0)$ be the triangulated subcategory of the category $\mathcal{D} \mathcal{M}_{F}^{\text {eff }}$ of effective motives over $F$ generated by the Tate motives $\mathbb{Q}(i), i \geq 0$. (Notice that Voevodsky works with the "homological" motives.) Let $\mathcal{D}_{T}(m, n)$ be the triangulated subcategory generated by the Tate motives $\mathbb{Q}(i), m \leq i \leq n$. Suppose $X=X_{P} \oplus X_{P}^{\prime}$. Let us show using induction on $n$ that if $X \in \mathcal{D}_{T}(0, n)$, then $X_{P} \in \mathcal{D}_{T}(0, n)$.

Step 1: $n=0$. The category $\mathcal{D}_{T}(0):=\mathcal{D}_{T}(0,0)$ is equivalent to the bounded derived category of $\mathbb{Q}$-vector spaces, which is known to be equivalent to the abelian category of graded $\mathbb{Q}$-vector spaces, so the statement follows.

Step 2. Let us construct functors

$$
\tau_{\leq n}: \mathcal{D}_{T}(\geq 0) \longrightarrow \mathcal{D}_{T}(0, n), \quad \tau_{>n}: \mathcal{D}_{T}(\geq 0) \longrightarrow \mathcal{D}_{T}(>n)
$$

and natural transformations $I d \longrightarrow \tau_{\leq n}, \tau_{>n} \longrightarrow I d$ such that for any $X \in \mathcal{D}_{\bar{T}}^{\geq 0}$ the sequence $\tau_{>n} X \longrightarrow X \longrightarrow \tau_{\leq n} X$ extends to a distinguished triangle

$$
\tau_{>n} X \longrightarrow X \longrightarrow \tau_{\leq n} X \longrightarrow \tau_{>n} X(1) .
$$

There is inner Hom in the category $\mathcal{D} \mathcal{M}_{F}^{\text {eff }}$, denoted by $\mathcal{H o m}_{\mathcal{D M}_{F}^{\text {eff }}}(*, *)$ (see 4.3.7 in $[\mathrm{V}])$ :

$$
\operatorname{Hom}_{\mathcal{D M}_{F}^{e f f}}(X \otimes Y, Z)=\operatorname{Hom}_{\mathcal{D M}_{F}^{e f f}}\left(X, \mathcal{H o m}_{\mathcal{D M}_{F}^{e f f}}(Y, Z)\right) .
$$


One has for $n \geq 0$

$$
\mathcal{H}_{o m_{\mathcal{D}}}^{\text {eff }}(\mathbb{Q}(n), \mathbb{Q}(n+m))=\mathbb{Q}(m) \text { if } m \geq 0 \text { and } 0 \text { otherwise. }
$$

Set

$$
\tau_{>n}(X):=\mathcal{H o m}_{\mathcal{D} \mathcal{M}_{F}^{\text {eff }}}(\mathbb{Q}(n+1), X) \otimes \mathbb{Q}(n+1) .
$$

The natural morphism $\tau_{>n}(X) \longrightarrow X$ is given by the canonical morphism

$$
\mathcal{H}^{\circ o m_{\mathcal{D}}^{\text {eff }}}(\mathbb{Q}(n+1), X) \otimes \mathbb{Q}(n+1) \longrightarrow X
$$

corresponding to the identity map on $\mathcal{H}_{\text {om }} \mathcal{M}_{F}^{\text {eff }}(\mathbb{Q}(n+1), X)$.

Further, set

$$
\tau_{\leq n}(X):=\mathcal{H} m_{\mathcal{D M}_{F}^{e f f}}\left(\mathcal{H} m_{\mathcal{D M}_{F}^{e f f}}(X, \mathbb{Q}(n)), \mathbb{Q}(n)\right) .
$$

Then there exists a natural morphism

$$
X \longrightarrow \mathcal{H} \mathrm{m}_{\mathcal{D} \mathcal{M}_{F}^{\text {eff }}}\left(\mathcal{H}_{\mathrm{om}} \operatorname{\mathcal {M}}_{F}^{\text {eff }}(X, \mathbb{Q}(n)), \mathbb{Q}(n)\right)
$$

provided by the identity element in

$$
\begin{gathered}
\operatorname{Hom}\left(X \otimes \mathcal{H o m}_{\mathcal{D} \mathcal{M}_{F}^{\text {eff }}}(X, \mathbb{Q}(n)), \mathbb{Q}(n)\right) \\
=\operatorname{Hom}\left(\mathcal{H o m}_{\mathcal{D} \mathcal{M}_{F}^{\text {eff }}}(X, \mathbb{Q}(n)), \mathcal{H o m}_{\mathcal{D} \mathcal{M}_{F}^{\text {eff }}}(X, \mathbb{Q}(n))\right) .
\end{gathered}
$$

It is easy to check that these two functors have all the needed properties.

Since $\tau_{>0}$ is a functor, we get a decomposition $X_{>0}=\left(X_{>0}\right)_{P} \oplus\left(X_{>0}\right)_{P}^{\prime}$. Consider the following diagram:

$$
\begin{array}{ccccccc}
\left(X_{>0}\right)_{P} & \stackrel{f_{1}}{\longrightarrow} & X_{P} & \stackrel{f_{2}}{\longrightarrow} & \text { Cone } & \stackrel{f_{3}}{\longrightarrow} & \left(X_{>0}\right)_{P}[1] \\
i_{1} \downarrow & & i_{2} \downarrow & & i_{3} \downarrow & & \downarrow i_{1}[1] \\
X_{>0} & \stackrel{g_{1}}{\longrightarrow} & X & \stackrel{g_{2}}{\longrightarrow} & X_{0} & \stackrel{g_{3}}{\longrightarrow} & X_{>0}[1] \\
j_{1} \downarrow & & j_{2} \downarrow & & j_{3} \downarrow & & \downarrow \\
\left(X_{>0}\right)_{P} & \stackrel{f_{1}}{\longrightarrow} & X_{P} & \stackrel{f_{2}}{\longrightarrow} & \text { Cone } & \longrightarrow & \left(X_{>0}\right)_{P}[1]
\end{array}
$$

Here $i_{2}: X_{P} \longrightarrow X$ is the natural morphism and $j_{2}: X \longrightarrow X_{P}$ is the projection onto the direct summand $X_{P}$, so $j_{2} \circ i_{2}=0$. Similarly $j_{1} \circ i_{1}=0$.

Lemma 5.11. For any $M \in \mathcal{D}_{T}(1, n)$ one has $\mathcal{H o m}_{\mathcal{D} \mathcal{M}_{F}}(M$, Cone $)=0$.

Proof. Let $m: M \longrightarrow$ Cone. It is easy to see using $\operatorname{Hom}_{\mathcal{D} \mathcal{M}_{F}}(\mathbb{Q}(i), \mathbb{Q}(0))=0$ for $i>0$ that for any $X_{0} \in \mathcal{D}_{T}(0)$ one has $\operatorname{Hom}_{\mathcal{D} \mathcal{M}_{F}}\left(M, X_{0}\right)=0$. So $i_{3} \circ m: M \longrightarrow X_{0}$ is zero. Therefore $i_{1}[1] \circ f_{3} \circ \mathrm{m}=g_{3} \circ i_{3} \circ \mathrm{m}=0$. Since $\left(X_{>0}\right)_{P}[1]$ is a direct summand of $X_{>0}[1]$, this implies that $m \circ f_{3}=0$. So there exists a morphism $m_{1}: M \longrightarrow X_{P}$ such that $m=f_{2} \circ m_{1}$. The composition $g_{2} \circ i_{2} \circ m_{1}: M \longrightarrow X_{0}$ is zero, so there exists $n: M \longrightarrow X_{>0}$ such that $g_{1} \circ n=i_{2} \circ m_{1}$. Then $j_{1} \circ n: M \longrightarrow\left(X_{>0}\right)_{P}$ is a map such that its composition with $f_{1}$ is $m_{1}$. This implies that $m=m_{1} \circ f_{2}=0$. The lemma is proved.

Lemma 5.12. The map $j_{3} \circ i_{3}-I d:$ Cone $\longrightarrow$ Cone equals zero. 
Proof. $\left(j_{3} \circ i_{3}-I d\right) \circ f_{2}=f_{2} \circ\left(j_{2} \circ i_{2}-I d\right)=0$ since $j_{2} \circ i_{2}=I d$. Therefore there exists a map $h:\left(X_{>0}\right)_{P}[1] \longrightarrow$ Cone such that $h \circ f_{3}=j_{3} \circ i_{3}-I d$. Since $\left(X_{>0}\right)_{P}[1] \in \mathcal{D}_{T}(1, n)$, one has $h \circ f_{3}=0$ by Lemma 5.11. The lemma is proved.

So Cone is a direct summand of $X_{0}$ and thus belongs to $\mathcal{D}_{T}(0)$ by Step 1 because $X_{0} \in \mathcal{D}_{T}(0)$. Since $\left(X_{>0}\right)_{P}$ belongs to $\mathcal{D}_{T}(1, n)$ by the induction assumption, we conclude that $X_{P} \in \mathcal{D}_{T}(0, n)$. The proposition is proved.

5. The abelian category of mixed Tate motives over a number field. The problem is the Beilinson-Soulé vanishing conjecture which guarantees that the negative Ext's are zero. However if $F$ is a number field, the vanishing conjecture follows from the results of Borel and Beilinson $([\mathrm{B} 2],[\mathrm{Bo} 2])$. So $\operatorname{Hom}_{\mathcal{D} \mathcal{M}}^{-i}(\mathbb{Q}, \mathbb{Q}(n))=0$ for all $i, n>0$. The category $\mathcal{D} \mathcal{M}_{F}$ is a subcategory of the derived category of sheaves with transfers (see $[\mathrm{V}]$ ), so we may apply part b) of Proposition 5.8. Therefore we can apply the "category machine" from s. 4.3 and get the abelian category $\mathcal{M}_{T}(F)$ of mixed Tate motives over a number field $F$ with all the needed properties. In particular we have the Hopf algebra $\mathcal{A}_{\bullet}(F)$ provided by the Tannakian formalism.

6. An application: A mixed Tate motive $m(Q, L)$. A pair $\left(\mathbb{P}^{n} \backslash Q, L\right)$ where $Q$ is a nondegenerate quadric and $L$ is a simplex provides an object $m(Q, L)$ in $\mathcal{D} \mathcal{M}_{F}$. Namely, for a subset $I=\left\{j_{1}<\ldots<j_{n-i}\right\}$ of $\{0,1, \ldots, n\}$ let $L(I):=L_{j_{1}} \cap L_{j_{2}} \cap \ldots \cap$ $L_{j_{n-i}}$ be the corresponding $i$-dimensional face of $L$ and $L^{Q}(I):=L(I) \backslash(Q \cap L(I))$. Let $L^{Q}(i):=\bigcup_{|I|=n-i} L^{Q}(I)$. Then $m(Q, L):=\operatorname{Hom}(\tilde{m}(Q, L), \mathbb{Q})$. Here

$$
\tilde{m}(Q, L):=L^{Q}(0) \longrightarrow L^{Q}(1) \longrightarrow \ldots \longrightarrow L^{Q}(n-1) \longrightarrow \mathbb{P}^{n} \backslash Q
$$

where the first group is sitting in degree 0 , and the differentials decrease the degree and are given by the usual rules in the simplicial resolution. According to Theorem 4.1.11 in [V] one has $M\left(P^{n}\right)=\bigoplus_{i=0}^{n} \mathbb{Q}(i)[2 i]$.

Lemma 5.13. Suppose $F$ is an algebraically closed field. Then $M(Q) \in \mathcal{D}_{T}(F)$.

Proof. Let $Z \subset X$ be a smooth subvariety of codimension $c>1$ in a smooth variety $X$ over $F$. Let $X_{Z}$ be the blow up of $Z$ at $X$. Then (3.5.3 in [V]) $M\left(X_{Z}\right)=$ $M(X) \oplus \bigoplus_{i=1}^{c-1} M(Z)(i)[2 i]$. Let $Q_{x}^{d}$ be the blow up of the quadric $Q^{d}$ of dimension $d$ at a point $x$. Then $M\left(Q_{x}^{d}\right)=M\left(P_{Q^{d-2}}^{d}\right)$. The lemma follows by induction using Proposition 5.10.

Therefore $m(Q, L) \in \mathcal{D}_{T}(F)$. So we can define an object

$$
M(Q, L):=H_{t}^{2 n-1}(m(Q, L)) \in \mathcal{M}_{T}(F) .
$$

Here $H_{t}$ is the cohomology with respect to the $t$-structure on $\mathcal{D}_{T}(F)$. In fact $H_{t}^{2 n-1}(m(Q, L))=m(Q, L)$ (since this is true in the Hodge realisation), but we will not use this. The mixed Tate motive $M(Q, L)$ has canonical framing. When $F=\overline{\mathbb{Q}}$ or $\mathbb{C}$ we can construct it by applying the Hodge realisation functor Hod from the abelian category of mixed Tate motives to the abelian category of mixed Hodge-Tate structures and using the framing on $\operatorname{Hod}(M(Q, L))$. Notice that $\operatorname{Hod}$ is an exact functor between the corresponding abelian categories sending the Tate objects to their counterparts, and the $\operatorname{End}(\mathbb{Q}(i))=\mathbb{Q}$ in both categories of motives and Hodge structures. (In general one can repeat the definition of the framing in the motivic setting.) 
7. Proof of Theorem 1.6: The final step. For any embedding $\sigma: F \hookrightarrow \mathbb{C}$ there is the Hodge realisation functor:

$$
\operatorname{Hod}_{\sigma}: \mathcal{M}_{T}(F) \longrightarrow \mathcal{H}_{T}
$$

It follows from the Borel theorem (injectivity of the regulator map on $K_{2 n-1}(F) \otimes \mathbb{Q}$ for number fields) that $\bigoplus_{\sigma} \operatorname{Hod}_{\sigma}$ induces an injective map on

$$
\bigoplus_{\sigma} H_{\sigma}: \operatorname{Ext}_{\mathcal{M}_{T}(F)}^{1}(\mathbb{Q}(0), \mathbb{Q}(n)) \hookrightarrow \bigoplus_{\sigma} \operatorname{Ext}_{\mathcal{H}_{T}(F)}^{1}(\mathbb{Q}(0), \mathbb{Q}(n)) .
$$

Let $\Sigma$ be the set of all complex embeddings of the number field $F$ in $\mathbb{C}$. Denote by $\mathcal{F}_{\Sigma} \mathcal{H}_{\bullet}$ the Hopf algebra obtained by the following procedure (it is the free coproduct of several copies of the Hopf algebra $\mathcal{H}_{\bullet}$ indexed by the set $\Sigma$ ). Let $L_{\bullet}^{\mathcal{H}}:=\frac{\mathcal{H}_{>0}}{\left(\mathcal{H}_{>0}\right)^{2}}$. It is a Lie coalgebra. Let $\left(L_{\bullet}^{\mathcal{H}}\right)^{\vee}$ be its dual.

Denote by $\mathcal{F}_{\Sigma}\left(L_{\bullet}^{\mathcal{H}}\right)^{\vee}$ the free product of several copies of the Lie algebra $\left(L_{\bullet}^{\mathcal{H}}\right)^{\vee}$ indexed by the set $\Sigma$. Then $\mathcal{F}_{\Sigma} \mathcal{H}_{\bullet}$ is the dual to the universal enveloping algebra of the Lie algebra $\mathcal{F}_{\Sigma}\left(L_{\bullet}^{\mathcal{H}}\right)^{\vee}$.

The Hodge realisation provides a diagram $I$ :

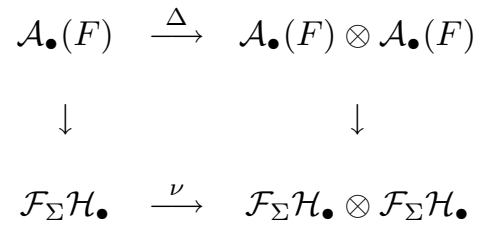

We claim that injectivity of (66) implies that the vertical arrows are embeddings. To prove this statement we translate it into the language of Lie algebras. We need to show that the natural morphism of the Lie algebras

$$
\mathcal{F}_{\Sigma}\left(L_{\bullet}^{\mathcal{H}}\right)^{\vee} \longrightarrow L_{\bullet}(F)
$$

is surjective. Since $H_{1}\left(\mathcal{F}_{\Sigma}\left(L_{\bullet}^{\mathcal{H}}\right)^{\vee}\right)=\bigoplus_{\sigma} H_{1}\left(\left(L_{\bullet}^{\mathcal{H}}\right)^{\vee}\right)$, the map on the $H_{1}$ 's induced by the Lie algebra morphism (67) is dual to the map (66), and so it is surjective since (66) is injective.

Further, there is a homomorphism $S_{\bullet} \longrightarrow \mathcal{A}_{\bullet}$ such that its composition with the Hodge realization map $\mathcal{A}_{\bullet} \longrightarrow \mathcal{F}_{\Sigma} \mathcal{H}_{\bullet}$ coincides with the homomorphism $S_{\bullet} \longrightarrow$ $\mathcal{F}_{\Sigma} \mathcal{H}_{\bullet}$. Here we use Lemma 5.13. Notice that we obviously can define framing on a mixed Tate motive over $\overline{\mathbb{Q}}$ using its Hodge realization. So there is a diagram $I I$ :

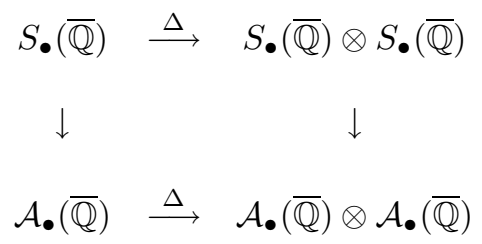

Let us show that this diagram is commutative using injectiveness of the vertical arrows of diagram $I$. (Abusing notations we denoted the comultiplication in the two different Hopf algebras by the same letter $\Delta$.) 
Let $x \in S_{\bullet}(\overline{\mathbb{Q}})$. Then there is a finite extension $F$ of $\mathbb{Q}$ such that $x$ and $\Delta(x)$ are defined over $F$. Consider the following diagram $I I I$ :

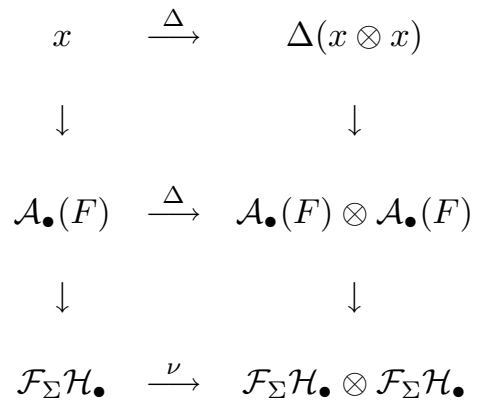

The "external" part of the diagram (i.e. the part given by the compositions of the vertical arrows and top and bottom horizontal arrows) is commutative thanks to the main results of Section 3 (especially Theorem 3.11). Both the external square and the bottom square are commutative, and the vertical arrows in the bottom square are injective, so the upper square is commutative.

This together with injectivity of vertical arrows in the bottom square of the diagram implies the commutativity of the upper square of the diagram. Theorem 1.6 follows from the commutativity of the upper square of the diagram. Indeed, the kernel of the middle horizontal arrow coincides with $K_{2 n-1}(F) \otimes \mathbb{Q}$ and the Beilinson regulator comes from the bottom square of that diagram.

The commutativity of the diagrams in Theorem 1.6 is provided by the results of this section and results of Section 4, especially s. 4.7 .

Let us summarize the key steps of the construction of the motivic extension corresponding to a hyperbolic manifold. Cutting a hyperbolic manifold $M^{2 n-1}$ on geodesic simplices, we get an element $s\left(M^{2 n-1}\right) \in \mathcal{P}\left(\mathcal{H}^{2 n-1}\right)$ (see s.2.1), which can be defined over $\overline{\mathbb{Q}}$. By Proposition 3.2, it belongs to the kernel of the Dehn invariant and so thanks to Theorem 5.2b) its image $\psi_{n}\left(s\left(M^{2 n-1}\right)\right)$ under homomorphism (60) lies in $\operatorname{Ker} \Delta$.

Therefore according to Theorem 3.11 the comultiplication $\tilde{\nu}$ of the corresponding $n$-framed Hodge-Tate structure $h \circ \psi_{\mathcal{H}}(n)\left(s\left(M^{2 n-1}\right)\right) \in \mathcal{H}_{n}$ is also 0 . Looking at the cobar complex $\mathcal{H}_{\bullet} \stackrel{\tilde{\nu}}{\longrightarrow} \mathcal{H}_{\bullet} \otimes \mathcal{H}_{\bullet} \longrightarrow \ldots$ for the Hopf algebra $\mathcal{H}_{\bullet}$ we see that $h \circ \psi_{\mathcal{H}}(n)\left(s\left(M^{2 n-1}\right)\right)$ is a 1-cocycle in this complex. According to Theorem 3.6 the cohomology class of this 1-cocycle provides us with an element of $\operatorname{Ext}_{M H S}^{1}(\mathbb{Q}(0), \mathbb{Q}(n))$ which is by construction of algebraic-geometrical origin.

\section{Remarks on Dehn complexes}

1. Dehn complexes in dimension 3 and Proposition 1.10. Recall the notation $S^{1}:=\mathbb{R} / \mathbb{Z}$.

Theorem 6.1 ([Du1], [DS1], [DPS]). The sequences

$$
\begin{gathered}
0 \longrightarrow H_{3}\left(S L_{2}(\mathbb{C})\right)^{-} \longrightarrow \mathcal{P}\left(\mathcal{H}^{3}\right) \stackrel{D_{3}^{H}}{\longrightarrow} \mathbb{R} \otimes S^{1} \longrightarrow H_{2}\left(S L_{2}(\mathbb{C})\right)^{-} \longrightarrow 0, \\
0 \longrightarrow H_{3}(S U(2)) \longrightarrow \mathcal{P}\left(S^{3}\right) \stackrel{D_{3}^{S}}{\longrightarrow} S^{1} \otimes S^{1} \longrightarrow H_{2}(S U(2)) \longrightarrow 0
\end{gathered}
$$

are exact. 
According to $[\mathrm{S}]$ and $[\mathrm{Sah} 3] H_{3} S L_{2}(\mathbb{C})=K_{3}^{\text {ind }}(\mathbb{C})$ modulo torsion. Further, $H_{2} S L_{2}(\mathbb{C})=K_{2}(\mathbb{C}), g r_{2}^{\gamma} K_{2}(\mathbb{C})=K_{2}(\mathbb{C})$ and $g r_{2}^{\gamma} K_{3}(\mathbb{C})=K_{3}^{\text {ind }}(\mathbb{C})$. Therefore the complex

$$
\mathcal{P}\left(\mathcal{H}^{3}\right) \stackrel{D_{3}^{H}}{\longrightarrow} \mathbb{R} \otimes S^{1}
$$

computes $K_{3}^{\text {ind }}(\mathbb{C})^{-}$modulo torsion and $K_{2}(\mathbb{C})^{-}$. Moreover, this complex is isomorphic to the "-" part of the Bloch complex:

$$
B_{2}(\mathbb{C}) \stackrel{\delta}{\longrightarrow} \bigwedge^{2} \mathbb{C}^{*}, \quad \delta\{x\}_{2}:=(1-x) \wedge x .
$$

(By the Matsumoto theorem Coker $\delta=K_{2}(\mathbb{C})$ and according to $[\mathrm{S}]$ and [Sah3] $\operatorname{Ker} \delta=K_{3}^{\text {ind }}(\mathbb{C})$ modulo torsion.)

Let $\mathcal{P}\left(\partial \mathcal{H}^{3}\right)$ be the scissors congruence group generated by the ideal tetrahedras in $\mathcal{H}^{3}$. Notice that $\partial \mathcal{H}^{3}=\mathbb{C P}^{1}$. Then the natural inclusion $\mathcal{P}\left(\partial \mathcal{H}^{3}\right) \hookrightarrow \mathcal{P}\left(\overline{\mathcal{H}}^{3}\right)=$ $\mathcal{P}\left(\mathcal{H}^{3}\right)$ is an isomorphism. This provides a construction of a map of complexes. The coincidence of differentials is not obvious and follows from calculations.

Finally, a rather involved construction (using the Hopf map $S^{3} \longrightarrow S^{2}$ ) of a homomorphism of the spherical complex

$$
\mathcal{P}\left(S^{3}\right) \stackrel{D_{3}^{S}}{\longrightarrow} S^{1} \otimes S^{1}
$$

to the "+" part of (71) was given in [DPS]. It is not an isomorphism since $\left(\mathbb{C}^{*} \otimes \mathbb{C}^{*}\right)^{+}=\mathbb{R}^{*} \otimes \mathbb{R}^{*} \oplus S^{1} \otimes S^{1}$, but induces isomorphisms on cohomologies ([SW], [DPS]). More precisely, there is a subgroup $S^{2}\left(S^{1}\right) \subset \mathcal{P}\left(S^{3}\right)$ (see [DPS]), and the Dehn invariant is injective on this subgroup. So taking the quotient we get a complex

$$
\mathcal{P}\left(S^{3}\right) / S^{2}\left(S^{1}\right) \stackrel{\bar{D}_{3}^{S}}{\longrightarrow} S^{1} \wedge S^{1}
$$

quasi-isomorphic to (72). The complex (73) is quasi-isomorphic to the "+"part of (71).

Proof of Proposition 1.10. $K_{n}^{M}(F)$ can be presented as a quotient of $\Lambda^{n} F^{*}$ by the subgroup generated by the Steinberg elements. Notice that

$$
\Lambda^{2} \mathbb{C}^{*}=\Lambda^{2} \mathbb{R} \oplus \Lambda^{2} S^{1} \oplus \mathbb{R} \otimes S^{1} .
$$

Let $S t_{2} \subset \Lambda^{2} \mathbb{C}^{*}$ be the subgroup generated by the Steinberg elements $(1-x) \wedge x$. Let

$$
S t_{2}^{-}:=\operatorname{Im} D_{3}^{H} \subset \mathbb{R} \otimes S^{1}, \quad S t_{2}^{+}:=\operatorname{Im} \bar{D}_{3}^{S} \subset \Lambda^{2} S^{1} .
$$

Since the cokernels of the maps $(70)$ and $(73)$ are isomorphic to $K_{2}(\mathbb{C})^{+}$and $K_{2}(\mathbb{C})^{-}$, we see that $S t_{2}^{ \pm} \subset S t_{2}$. Moreover, we have an isomorphism

$$
\frac{\Lambda^{2} S^{1}}{S t_{2}^{+}} \oplus \frac{\mathbb{R} \otimes S^{1}}{S t_{2}^{-}}=K_{2}(\mathbb{C})=\frac{\Lambda^{2} \mathbb{R} \oplus \Lambda^{2} S^{1} \oplus \mathbb{R} \otimes S^{1}}{S t_{2}} .
$$

This means that the canonical projection $\Lambda^{2} \mathbb{C}^{*} \longrightarrow \Lambda^{2} \mathbb{R}$ provides an isomorphism

$$
\frac{S t_{2}}{S t_{2}^{+} \oplus S t_{2}^{-}} \longrightarrow \Lambda^{2} \mathbb{R}
$$

(one can also check its surjectivity directly). Therefore the canonical maps

$$
\Lambda^{n} S^{1} \longrightarrow K_{n}^{M}(\mathbb{C})^{+} \quad \text { and } \quad \mathbb{R} \otimes \Lambda^{n-1} S^{1} \longrightarrow K_{n}^{M}(\mathbb{C})^{-}
$$


are surjective. Indeed, write

$$
\Lambda^{n} \mathbb{C}^{*}=\Lambda^{n} S^{1} \quad \oplus \quad \mathbb{R} \otimes \Lambda^{n-1} S^{1} \quad \oplus \quad \Lambda^{2} \mathbb{R} \otimes \Lambda^{n-2} S^{1} \quad \oplus \quad \ldots
$$

Proposition 1.10 is proved.

\section{The structure of the complexes $S(\mathbb{C} ; n) \bullet$ for $n \leq 3$.}

Conjecture 6.2. Let $F$ be an algebraically closed field. Then there exist canonical homomorphisms of complexes

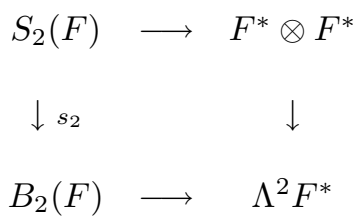

and

$$
\begin{aligned}
& S_{3}(F) \quad \longrightarrow \quad F^{*} \otimes S_{2}(F) \oplus S_{2}(F) \otimes F^{*} \quad \longrightarrow \quad F^{*} \otimes F^{*} \otimes F^{*} \\
& \downarrow s_{3} \quad \downarrow \text { id } \otimes s_{2}+s_{2} \otimes \text { id } \quad \downarrow \\
& B_{3}(F) \quad \longrightarrow \quad B_{2}(F) \otimes F^{*} \quad \longrightarrow \quad \Lambda^{3} F^{*}
\end{aligned}
$$

which induce isomorphisms on homology modulo torsion. In the case $F=\mathbb{C}$ the composition

$$
\mathcal{P}\left(\mathcal{H}^{2 n-1}\right) \longrightarrow S_{n}(\mathbb{C}) \stackrel{s_{n}}{\longrightarrow} B_{n}(\mathbb{C}) \stackrel{\mathcal{L}_{n}}{\longrightarrow} \mathbb{R} \quad(n=2,3)
$$

should coincide with the volume homomorphism.

So the homomorphisms $s_{n}(n=2,3)$ will lead to explicit formulas for volumes of noneuclidean simplices via classical di and trilogarithms (compare with the work of R. Kellerhals [K1]).

Here the group $B_{2}(F)$ was introduced in 1.4 and the group $B_{3}(F)$ is an explicit version of the group $\mathcal{B}_{3}(F)$ from s.1.5; see [G2]. The differentials in the $B$-complexes are defined by the formulas $\{x\}_{2} \longmapsto(1-x) \wedge x$ and $\{x\}_{3} \longmapsto\{x\}_{2} \otimes x,\{x\}_{2} \otimes y \longmapsto$ $(1-x) \wedge x \wedge y$. A similar result, of course, should be valid for any algebraically closed field.

The kernel of the maps $s_{2}, s_{3}$ should be equal to $S_{1}(F) * S_{1}(F)$ and $S_{1}(F) * S_{2}(F)$, where $*$ denotes the product in the algebra $S_{\bullet}(F)$. This follows from the PoincaréBirkhoff-Witt theorem for the Hopf algebra $S_{\bullet}(F)^{*}$.

The most interesting problem here is a construction of an explicit homomorphism $S_{3}(F) \longrightarrow B_{3}(F)$ and $S_{2}(F) \longrightarrow B_{2}(F)$ which commutes with the differentials. These homomorphisms should have a very nice geometrical description. The homomorphism $S_{2}(F) \longrightarrow B_{2}(F)$ is easy to define for simplices whose vertices are on the quadric $Q$. Namely, $Q=P^{1} \times P^{1}$, so such a simplex is defined by a 4 -tuple $\left[\left(x_{1}, y_{1}\right),\left(x_{2}, y_{2}\right),\left(x_{3}, y_{3}\right),\left(x_{4}, y_{4}\right)\right]$ of points of $P^{1} \times P^{1}$. Its image in $B_{2}(F)$ should be $\left\{r\left(x_{1}, x_{2}, x_{3}, x_{4}\right)\right\}_{2}-\left\{r\left(x_{1}, x_{2}, x_{3}, x_{4}\right)\right\}_{2}$.

3. Reflections about euclidean Dehn complexes and mixed Tate motives over the dual numbers. The following conjecture expresses a general expectation about the kernel of the euclidean Dehn invariant. It tells us that the Dehn invariant and the volume homomorphism separate all elements of the euclidean scissors congruence group. 
Conjecture 6.3. $\operatorname{Ker} D^{E}=\mathbb{R}$.

Let $\Omega_{\mathbb{R} / \mathbb{Q}}^{i}$ be the Kähler differential $i$-forms of the field $\mathbb{R}$.

Question 6.4. Is it true that $H^{i}\left(\mathcal{P}_{E}^{\bullet}(n)\right) \otimes \mathbb{Q}=\Omega_{\mathbb{R} / \mathbb{Q}}^{i-1}$ ?

For $n=2$ this is the beautiful theorem of Sydler, later reproved by Jessen and Thorup and Dupont-Sah [DS2].

Notice that $\Omega_{\mathbb{R} / \mathbb{Q}}^{0}=\mathbb{R}$, so Conjecture 6.3 is equivalent to the positive answer to this question for $i=1$ and arbitrary $n$.

Here is a hypothetical motivic explanation supporting Question 6.4. One could think of euclidean geometry as of degeneration of hyperbolic geometry. Namely, consider the Cayley model of the hyperbolic geometry inside of the sphere $Q_{\varepsilon}$ given by the equation $\varepsilon\left(x_{1}^{2}+\ldots+x_{n}^{2}\right)=x_{0}^{2}$ in homogeneous coordinates. Then in the limit $\varepsilon \longrightarrow 0$ we will get the euclidean geometry in $\mathbb{R}^{n}$. Now let $\varepsilon$ be a variable with $\varepsilon^{2}=0$. Then one should imagine that $H\left(Q_{\varepsilon}, M\right):=H^{n}\left(\mathbb{P}^{n} \backslash Q_{\varepsilon}, M\right)$ is a variation (or better to say a deformation because it should split at $\varepsilon=0$ ) of mixed Tate motives over $S p e c \mathbb{R}[\varepsilon]$. Notice that right now there is not even a hypothetical definition of the category of mixed motives or mixed Hodge structures over a scheme with nilpotents. The euclidean Dehn invariant should have a natural interpretation in the language of deformations of mixed Hodge structures over $S p e c \mathbb{R}[\varepsilon]$ similar to the one for noneuclidean Dehn invariants given in Section 3.

Let $D_{T}(\varepsilon)$ be the hypothetical category of mixed Tate motives over $\operatorname{Spec} \mathbb{R}[\varepsilon]$ which split over $S p e c \mathbb{R}$. It is natural to conjecture that

$$
\operatorname{Ext}_{D_{T}(\varepsilon)}^{i}(\mathbb{Q}(0), \mathbb{Q}(n))=\operatorname{Ker}\left(g r_{n}^{\gamma} K_{2 n-i}(\mathbb{R}[\varepsilon]) \longrightarrow g r_{n}^{\gamma} K_{2 n-i}(\mathbb{R})\right) \otimes \mathbb{Q} .
$$

One can compute the right hand side using comparison with the cyclic homology ([Ca], [FT], [Goo]) and get the following theorem (I am indebted to B. Tsygan for sketching the proof for me):

Theorem 6.5. $\operatorname{Ker}\left(g r_{n}^{\gamma} K_{2 n-i}(\mathbb{R}[\varepsilon]) \longrightarrow g r_{n}^{\gamma} K_{2 n-i}(\mathbb{R})\right) \otimes \mathbb{Q}=\Omega_{\mathbb{R} / \mathbb{Q}}^{i-1}$.

Therefore one should get a canonical map $H^{i} \mathcal{P}_{E}^{\bullet}(n) \longrightarrow \Omega_{\mathbb{R} / \mathbb{Q}}^{i-1}$. I hope that it is an isomorphism.

\section{ACKNOWLEDGEMENTS}

This work was essentially done during my stay in MPI(Bonn) and MSRI(Berkeley) in 1992-1993 and supported by NSF Grant DMS-9022140. I am grateful to these institutions for hospitality and support. Later I was also supported by NSF Grants DMS-9500010 and DMS-9800998.

I am indebted to M. Kontsevich and D.B. Fuchs for useful discussions, and to L. Positselsky and V. Voevodsky for help in writing section 5.3. I am very grateful to A. Borel who read a preliminary version of this paper and pointed out many misprints and some errors. Finally I am very grateful to the referee who pointed out some misprints and gaps and made a lot of useful comments and suggestions.

\section{REFERENCES}

[Ao] Aomoto K.: Analytic structure of Schlafi function, Nagoya Math. J. 68 (1977), 1-16. MR 58:27972

[B1] Beilinson A.A.: Height pairings between algebraic cycles, Lecture Notes in Math. N. 1289, (1987), p. 1-26. MR 89h:11027 
[B2] Beilinson A.A.:Higher regulators and special values of L-functions VINITI, 24 (1984), 181-238. English translation: J.Soviet Math., 30 (1985), 2036-2070. MR 86h:11103

[BBD] Beilinson A.A., Bernstein J., Deligne P.: Faisceaux pervers, Asterisque 100, 1982. MR 86g:32015

[BD] Beilinson A.A., Deligne P.: Motivic polylogarithms and Zagier's conjecture. Preprint to appear.

[BD1] Beilinson A.A., Deligne P.: Interprétation motivique de la conjecture de Zagier. Proc. of AMS Research Summer Conference "Motives", Symposium in Pure Mathematics, vol 55, part 2. MR 95a: 19008

[BMS] Beilinson A.A., Macpherson R.D. Schechtman V.V: Notes on motivic cohomology, Duke Math. J., 1987 vol 55 p. 679-710. MR 88f:14021

[BGSV] Beilinson A.A., Goncharov A.B., Schechtman V.V, Varchenko A.N.: Aomoto dilogarithms, mixed Hodge structures and motivic cohomology of a pair of triangles in the plane, The Grothendieck Feschtrift, Birkhauser, 1990, p 131-172. MR 92h:19007

[Bl1] Bloch S.: Algebraic cycles and higher K-theory, Advances in Math. 61, n 3, (1985), 65-79. MR 88f: 18010

[Bl2] Bloch S.: Moving lemma for Higher Chow groups, Journal of Algebraic geometry (1995). MR 96c: 14007

[B13] Bloch S.: Applications of the dilogarithm function in Algebraic K - theory and algebraic geometry, Symp. on Alg. Geom., Kyoto, 1977, Kinokuniya Bookstore, Tokyo, 1978. MR 82f: 14009

[B14] Bloch S.: Function theory of polylogarithms, Chapter 12 in "Structural properties of Polylogarithms", L. Lewin, Editor, Math. Surveys and monographs, vol. 37; AMS, 1991. CMP 92:07

[Bo] Böhm J.: Inhaltsmessung in $\mathbb{R}^{5}$ constanter Krümmung, Arch.Math. 11 (1960), 298-309. MR 22: 12448

[Bo1] Borel A.: Cohomologie des espaces fibrés principaux, Ann. Math. 57 (1953), 115-207. MR 14:490e

[Bo2] Borel A.: Cohomologie de $S L_{n}$ et valeurs de fonctions zêta aux points entiers, Annali Scuola Normale Superiore Pisa 4 (1977), 613-636. MR 58:22016

[Ca] Cathelineau J.-L.: $\lambda$-operations structure in algebraic $K$-theory and cyclic homology, $K$-theory 4, N6, 591-606 (1991). MR 92k:19003

[Ch] Chern S.-S.: A simple intrinsic proof of the Gauss-Bonnet formula for closed Riemannian manifolds, Ann. of Math., vol 45, 1944, N 4. MR 6:106a

[Du1] Dupont J.: Algebra of polytops and homology of flag complexes, Osaka J. Math. 19 (1982), 599-641. MR 85f:52014

[Du2] Dupont J.: The dilogarithm as a characteristic class for a flat bundle, J. Pure and Appl. Algebra, 44 (1987), 137-164. MR 88k:57032

[DPS] Dupont J., Parry W., Sah H.: Homology of classical groups made discrete.II. $H_{2}, H_{3}$, and relations with scissors congruences, J. Pure and Appl. Algebra, 113, 1988, p.215-260. MR 89h:22019

[DS1] Dupont J., Sah H.: Scissors congruences II, J. Pure and Appl. Algebra, v. 25, 1982, p. 159-195. MR 84b:53062b; MR 84m:53060

[DS2] Dupont J., Sah H.: Homology of Euclidean groups of motion made discrete and Euclidean scissors congruences, Acta Math. 164 (1990) 1-24. MR 91b:22016

[EP] Epstein D.B.A., Penner R.: Euclidean decomposition of non-compact hyperbolic manifolds, J.Diff. Geom. 27 (1988), 67-80. MR 89a:57020

[FT] Feigin B, Tsygan B.: Additive K-theory, Lect. Notes in Math. 1289. MR 89a:18017

[G1] Goncharov A.B.: Polylogarithms and motivic Galois groups, Proc. of AMS Research Summer Conference "Motives", Symposium in Pure Mathematics, vol 55, part 2, 43-97. MR 94m:19003

[G2] Goncharov A.B.: Geometry of configurations, polylogarithms and motivic cohomology, Advances in Mathematics, vol. 144, N2, 1995. 197-318. MR 96g:19005

[G3] Goncharov A.B.: Hyperlogarithms, multiple zeta numbers and mixed Tate motives, Preprint MSRI 058-93, June 1993.

[G4] Goncharov A.B.: Mixed elliptic motives. Proceedings of the 60-th Durham Symposium "Galois groups in arithmetic and algebraic geometry". (1998).

[G5] Goncharov A.B.: Geometry of polylogarithms and regulators, To appear. 
[G6] Goncharov A.B.: Analytic motivic complexes and characteristic classes, To appear.

[Goo] Goodwillie T.G.: Algebraic K-theory and cyclic homology, Annals of Math. 24 (1986) 344-399. MR 88b:18008

[H] Hanamura M.: Preprint MPI 1996.

[K1] Kellerhals, R.: On the volume of hyperbolic 5-orthoschemes and the trilogarithm, Comm. Math. Helv., 67 (1992), 648-663 MR 93g:52006

[K2] Kellerhals, R.: Volumes in hyperbolic 5-space, GAFA, 5 (1995), 640-667. MR 96g:52011

[L] Levine, M.: Motivic cohomology and algebraic cycles: a categorical construction, I, Preprint 1993.

[ML] Mac Lane, S.: Homology, Springer-Verlag, 1963. MR 28:122

[M1] Milnor J.: Computation of volume, Chapter 7 in [Th].

[Mu Müller P.: Über Simplexinhalte in nichteuklidischen Räumen, Dissertation Universität Bonn, 1954.

[NZ] Neumann W., Zagier D.: Volumes of hyperbolic three-manifolds, Topology 24 (1985), 307-331. MR 87j:57008

[P] Positselsky L.: Some remarks on triangulated categories, Preprint 1995.

[Ra] Raghunatan M.S. :Discrete subgroups of Lie groups, Springer Verlag, 1972.

[R] Ramakrishnan D.: Regulators, algebraic cycles and values of L-functions, Contemporary Math., vol 83, 183-310. MR 90e:11094

[Sah1] Sah H.: Hilbert's third problem: scissors congruences, Research notes in mathematics 33, Pitman Publishing Ltd., San-Francisco-London-Melbourne, 1979. MR 81g:51011

[Sah2] Sah H.: Scissor congruences, I: The Gauss-Bonnet map, Math. Scand. 49 (1981), 181210. MR 84b:53062a; MR 85b:53059

[Sah3] Sah H.: Homology of classical groups made discrete. III, J.pure appl. algebra 56 (1989) 269-312. MR 91k:19002

[SW] Sah H., Wagoner J.B: Second homology of Lie groups made discrete, Comm. Algebra 5(1977), 611-642. MR 58:31128

[Sch] Schläfli: On the multiple integral $\int^{n} d x d y \ldots d z$, whose limits are $p_{1}=a_{1} x+b_{1} y+\ldots+$ $h_{1} z>0, p_{2}>0, \ldots, p_{n}>0$, and $x^{2}+y^{2}+\ldots+z^{2}<1$, in Gesammelte Mathematische Abhandlungen II, pp. 219-270, Verlag Birkhauser, Basel, 1953. MR 14:833p

[S] Suslin A.A.: $K_{3}$ of a field and Bloch's group, Proceedings of the Steklov Institute of Mathematics 1991, Issue 4. MR 91k:19003

[Th] Thurston W.:Geometry and topology of 3-manifolds, Princeton University, 1978.

[V] Voevodsky V.: Triangulated category of motives over a field, Preprint 1994.

[Z1] Zagier D.: Polylogarithms, Dedekind zeta functions and the algebraic K-theory of fields. Arithmetic Algebraic Geometry (G.v.d.Geer, F.Oort, J.Steenbrink, eds.), Prog. Math., Vol 89, Birkhauser, Boston, 1991, pp. 391-430. Proceedings of the Texel conference on Arithmetical Algebraic Geometry, 1990. MR 92f:11161

[Z2] Zagier D.: The remarkable Dilogarithm, J.Math. and Phys. Soc. 22 (1988), 131-145. MR 89f:33024

[W] Wang H.C.: Topics in totally discontinuous groups, in Symmetric spaces, Boothby-Weiss, Editors, New York, 1972.

Department of Mathematics, Brown University, Providence, Rhode Island 02912

E-mail address: sasha@math.brown.edu 